
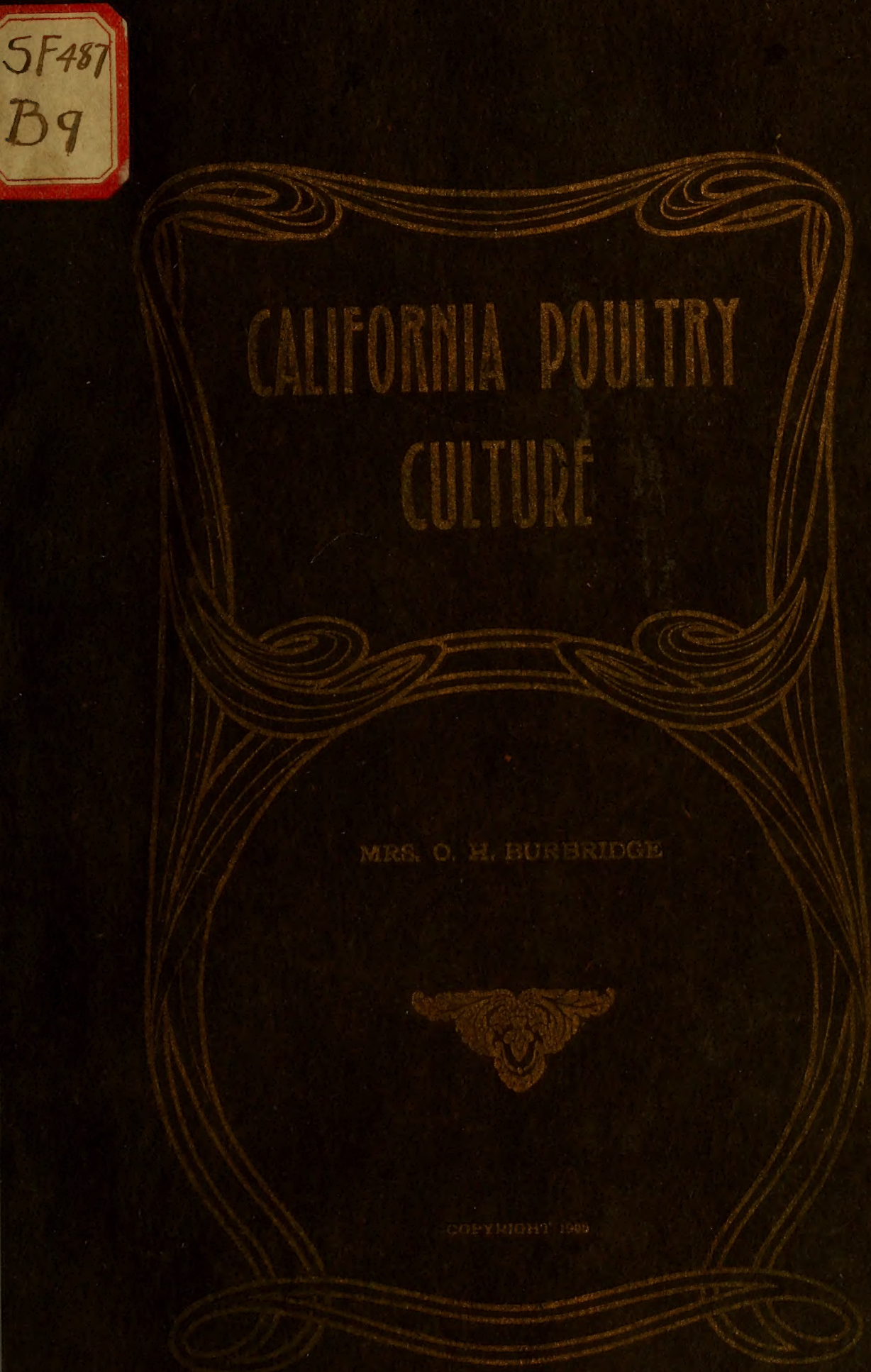






\section{CALIFORNIA POULTRY CULTURE}

\section{MRS. O. H. BURBRIDGE}

Editor PACIFIC FANCIER

Los Angeles, California 

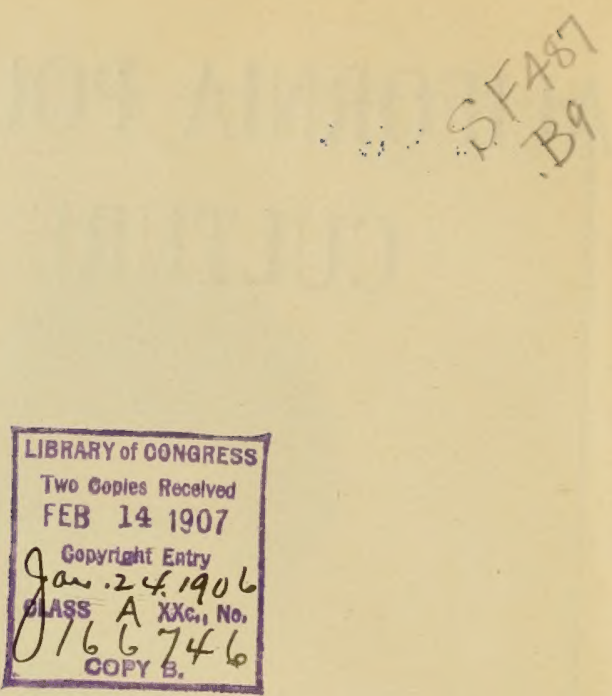

0
$\cdots$ 
CONTENTS.

California as a Poultry Raising State........... 5-7

Suitable Poultry Houses and Fixtures............ 8-2I

Care of the Breeding Stock............... 22-25

Incubation in California.................... 26-29

Care of the Chicks...................... 30-33

Care of Birds during the Moult.............. 34-36

Profitable Egg Ranching.................. 37-4I

Poultry Plant for Breeding Stock and Eggs for Hatching 42-5I

Crate Fattening of Chickens. . . . . . . . . . . . $\quad 5^{2-56}$

Caponizing .......................... 57-6

Lice and Mites........................ $62-65$

Diseases and Cures.................... $66-86$

Starting a Poultry Ranch on small Capital......... $\quad 87-89$

A Beginners Experience..................... $90-92$

Questions and Answers.................. 93-97

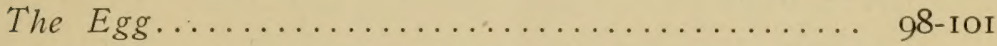

Management during Summer Months........... I02-I05

Breeds Best Adapted for California............. I06-I5I

Turkeys in California...................... I52-I 56

Ducks in California..................... I57-16 I

The Goose in California................. 162-165

Pigeons for Profit................... I66-I77 



\section{Introduction.}

The Editor of this little book has for a long time realized that a volume on California Poultry Culture was necessary to show our Eastern and Northern friends what could be done in the line of poultry breeding in this state. She has been for some years writing articles for poultry magazines, and for nearly two years past has been editor and manager of the largest poultry magazine west of Chicago, so has been able by the volume of correspondence from all points in the United States, England, Scotland, Australia and Hawaii to know what the people most desire to ascertain in regard to this subject. Mrs. Burbridge writes from the practical standpoint also, being owner and manager of one of the largest breeding-stock ranches in the West, namely Orpington Ranch, which is well known throughout the States. She has, while the ranch was growing, done every portion of the work, thus being able to speak from the practical working standpoint and not solely theoretically as so many of the authors of poultry books do. She has compiled this little volume from her own experience and from the best authorities in the West on the various subjects she has considered of most vital necessity to the new breeder. 



\section{California As a Poultry Raising State.}

\section{CLIMATIC CONDITIONS.}

California is the poultryman's Paradise. For three hundred and sixty days out of the three hundred and sixtyfive in our winterless year the birds can be out of doors scratching and singing instead of moping in cold, comfortless quarters as in the East and North. Here there is no necessity of expensive buildings to keep out the cold, no big coal and wood bills for artificial heat, never a frosted comb, and quantities of eggs in the winter. There is always plenty of sunshine, and everyone knows what a germicide the sun is. Many California poultry ranches have their houses so built that they can be opened to the rays of the sun every day and rarely are troubled with vermin of any sort. In the Coast towns there is always a cool breeze and in any part of California one can be comfortable out of the sun's direct rays, so if one lacks fruit or shade trees in the chicken yards a cheap shade structure can' be built and the birds made perfectly comfortable during the hot months.

There is no month in the year when a green crop cannot be raised; consequently the health of the bird is much better when kept on short green rations during the Eastern winter months. Nany of the most successful ranches run poultry in conjunction with fruit growing, the birds assisting in keeping down the insects and enriching the soil, thus helping the orchard, and the trees making the rery necessary shade and giving an occasional feed of cooling fresh fruit to the poultry.

In this land of sunshine Mother Nature dowers the children of man with that best of heritage, good health. It is not an exaggeration to say that the man or woman who is so fortunate as to live in California and who works or plays out of doors is practically immune from all the illness that flesh is heir to, and, with this greatest of all blessings added to the best climate on earth, a country full of ardent fanciers, one of the best egg and poultry markets in the States, a man or woman is a mighty poor manager who cannot make more than a good living out of poultry.

California sends over five million dollars yearly to the Middle West and Eastern States for market poultry, to say nothing of what is sent to the owners of fine breeding plants 
in the East and Europe. With the rapid growth of the cities this demand is becoming greater all the time and it is high time for the California rancher to grow more poultry. The ranches devoted to market poultry and eggs obtain a better average price in California than ony other State in the Union.

Of course I speak of the up-to-date ranchers who make their goods attractive, which is so easily and cheaply done, and often spells the word "success" instead of "failure."

California is the ideal State for poultry raising, yet it cannot supply half the demand of its own people, let alone the rapidly growing demands from the Pacific islands and the Orient.

Poultry raising especially appeals to the average Easterner who comes to California, for it gives him the means of making both ends meet for the first few years after he starts in the new life. Many of those who come to this State from the East do so because of bad health or because they have worn themselves down with arduous work. These men and women are desirous of entering some occupation which will give them plenty of outdoor exercise, without being too strenuous. Poultry raising does not demand a large amount of capital to start with, and there is no occupation that one can engage in that is more healthful. If a man or woman is strong enough for outdoor employment, caring for a handsome flock of fowls is a most pleasurable business as well as a most profitable one.

There prevails a widespread opinion in many parts of the East that poultry in California is subject to strange diseases, to attacks of vermin and to many other evils. This fallacy is one that has been exploded so often that it seems out of place again to contradict it. If there is a place on the face of the earth where poultry thrives better than in California that place is yet to be discovered.

So far as the commercial end of poultry raising in California is concerned, an ample income for an average family can be realized from five acres or less within twelve months from the start. The failures in the poultry business in California are due either to ignorance or shiftlessness.

There are three things which are absolutely necessary to success in the poultry business anywhere, and they are as necessary in California as anywhere else. These are industry, perseverance and cleanliness. They are prime essentials, and without them it is unwise to go into the business of raising chickens. To the man or woman with small capital the same difficulties present themselves as in other lines of business, making it necessary for one to begin modestly or carry a mortgage for a time, but there is no other business 
in which a man or woman can get as good returns for his money as in the poultry business.

If properly managed a farm of five acres and I 500 hens will keep the average family, a horse and a cow. Figuring from the market standpoint the ranch should clear $\$ 3000$ a year. It is estimated that the lowest net profit of the eggs will be $\$ 2.00$ a year for each hen, it will cost an average of $\$ 1.00$ per year to feed each of the flock. This calculation does not take into account the young stock for it is estimated that the sale of the young cockerels provide for the pullets which go to increase the flock, while the sale of the old hens after the third year will increase the profit of the place.

There are numerous instances in California where fortunes have been made from a start of a few dollars. But to the ordinary man or woman who would like to go into the business the results obtained by people of small means are of more interest. All over the State may be found people who are making good livings out of their flocks of fowls. They have started in a small way and increased their flocks gradually until now they have enough to bring them an income sufficient to provide them with all the comforts of life and many of the luxuries as well. In California are to be found many women who own small poultry farms, and who are leading independent lives, filled with health and happiness. These women came to the country broken in health and poor in purse, but they have recovered their health and are now enjoying the fruits of their labor.

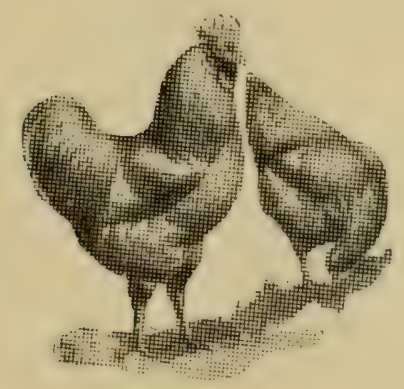




\title{
Suitable Poultry Houses and Fixtures.
}

\author{
IMPORTANCE OF PROPER HOUSING.
}

That the housing is very important, all must admit, for upon this depends a great deal of the success or failure of the venture. Nothing is so detrimental to the development of chickens as close and crowded houses, and only those who have opened a crowded and badly-ventilated house a few hours after the birds have gone to roost can fully realize how unwholesome foul air is, and yet it must be borne in mind that colds, roup, bronchitis and many other ailments are often caused by cracks and holes in the roosting quarters.

Good poultry houses may be summed up in two classes, one being the tight house with perfect ventilation at the bottom, and the other being the "free air" open or semi-open fronted house. Either of these is good if the principle is carried out correctly. Perhaps it may be necessary to explain what is meant by the open-fronted house, and we think a plan will be more easily understood than any other explanation.

\section{THE OPEN-FRONT HOUSE.}

Commence at the floor, which should be of well-tamped earth so that a sprinkling of sand or other dry matter can be spread over the surface and swept out when necessary. This is much better than digging up the inside of the house, for when it has been dug two or three times the whole mass becomes contaminated, whereas a few inches of scratching material can be removed very easily when foul.

The back (I) and (2) and ends (I), (3) and (2), (4) are closed. The front, which should face south, is closed only from (4)-(6), (3)-(7) is wired in, (7), (6) is the door, the perches are $(5),(8)$.

It will be seen that any wind can only reach the birds from point (3) and only then when they are roosting at point (5). Close observation has proved to us that on windy or cold nights they will retreat to point (3) and on sultry nights spread themselves to point (5). Here we have pure air and no draughts, provided the house has been built of such material as will make the sides, back and roof good and tight. Re-sawed redwood or even shakes, covered with any of the various building papers or clean felting that are on the market. Still more shelter can 
be had by using a canvas or burlap screen when necessary from (3) to (6). In such a house as above described it will be seen that the chickens can, at the will of the poultry keeper, be kept in at any time that it may be found necessary, and if during the wet days a few bushels of rough scratching litter be thrown on the floor in which they can be fed, the birds will not suffer.

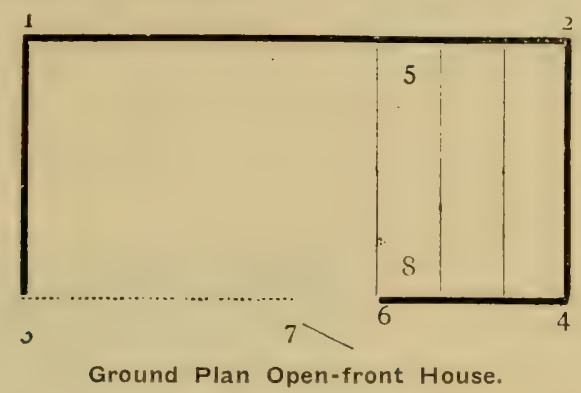

If one does not care for the open-fronted method of housing, front space from (3) to (7) may be boarded, also the door (7) to (6), two good ventilators placed one at each end of the house at the lowest point will give good ventilation and will make a capital house. We favor the open fronts ourselves, however.

\section{THE SCRATCHING SHED HOUSE.}

A fowl, like a man, must be occupied in order to be happy and useful. An over-fat drone female is both a poor egg producer and an easy prey to poultry diseases. Even if they do produce a few eggs in a season, the chicks hatched from them seldom amount to much, either as to vitality or productiveness.

With these facts before us, naturally the question was how best to keep our fowls busy and in proper shape to produce eggs that would hatch strong chicks. After many experiments we decided that birds kept under the yarding system, which we believe is the only way to get the best results from breeding birds, must be provided with scratching material in which they are required to work for all of their grain food. In order to save both yard and house room, we used a combined scratching and roosting house (a drawing of which is given herewith), which has proven very satisfactory in our yards and will suit the conditions of other California poultry raisers.

This house is 6 feet high in front, 4 feet in rear, floor space $8 \times 8$ feet, with door in end 2 feet wide; no dropping board is used but a IXI 2 board is placed on end, eighteen inches from rear of wall. (This to keep the scratching material away from the dropping floor.) A perch is placed about the center of the dropping floor space about twenty-four inches from the ground. 
the number of houses and runs may be increased without tearing down a whole lot of their early labors. It is much easier to commence right, and even if you do not enlarge your plant as time goes on, nothing is lost; but should you require to do so, if you start right, much is gained. When buying or renting a yard or ranch first get your measurements, decide how much you are going to let the chickens have and how much the garden, orchard or any other domestic matter is to occupy. When you have the measurements of the chicken portion, if it be a small lot, get the square measurements of it, divide by fifteen and that roughly will tell you how many birds your ground will carry; or, in other words, each bird requires fifteen square feet. By this it will be seen that on a lot of IOO by 25 about I2O chickens can be kept if perfect cleanliness be carried out. We

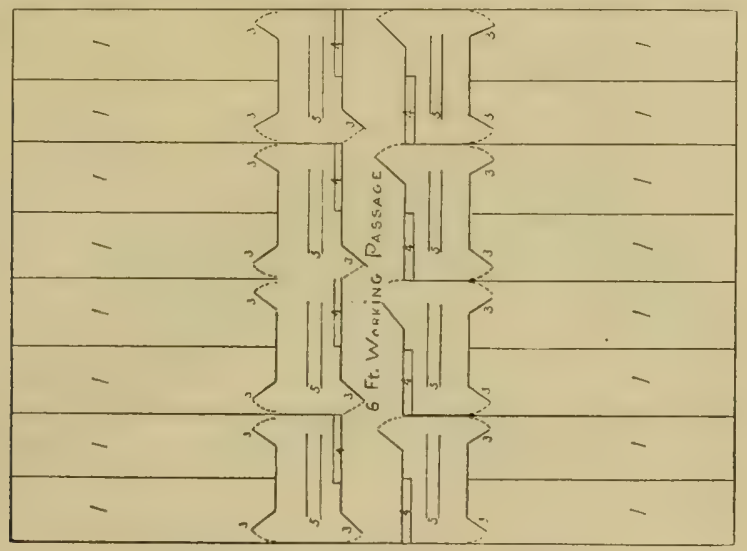

Diagram Complete Poultry Plant.

clo not say that this is the maximum amount that may be kept, but do not recommend more. If it is possible for them to get away to a grass run occasionally, many more may be kept.

After having decided the type, size and other things in connection with the house and yards, the next thing to be considered is the fencing. As to what is best and cheapest, depends upon tircumstances. Lathing placed diagonally is a pretty and cheap fence, but not very durable. The usual fencing is the ordinary chicken wire which is fairly cheap and the most practical. When fencing the yards it is most important that the bottom should have board or other material so that the birds cannot see each other. It prevents the cocks from fighting and the youngsters from fretting. About five feet is the usual height for the fencing; three feet will suffice for Cochins and Dorkings, about five feet for Orpingtons and Rocks, but it may be necessary to have six feet for Games, Leghorns and other breeds. 
The following diagram is a suggestion for a very complete plant, and though it will cost a little more than one usually cares to go into at the commencement, it is not in the least extravagant, for the amount of time saved by having such compact yards will soon pay the poultry keeper back. 'Taking the numbers consecutively, the explanation is as follows: Number I the runs,

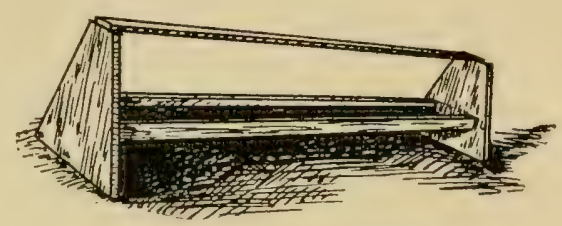

Trough for Mash or Dry Feeding.

2 the houses, 3 the doors, 4 the nest boxes, 5 the perches. It will be seen that there is a six-foot passage in which to do the necessary work, such as cleaning out, watering, disinfecting, etc. It will also be seen that we have given two yards to each house. This is a point that we would insist upon above everything else. The birds can be kept in excellent health in this manner. From recent experiments that we have made we find that a crop of wheat or barley can be produced in four weeks sufficient to cleanse the ground and give the birds a nice green patch which will last quite a little while. The soft grain is also an inducement for them to scratch which gives them good exercise. In the meantime the spare yard is again set to barley or wheat ready for the next move. The perches can be made to swing upon hinges, so that the interior of the house is quite clear, and on wet and cold days, if some good, rough litter be placed inside, can

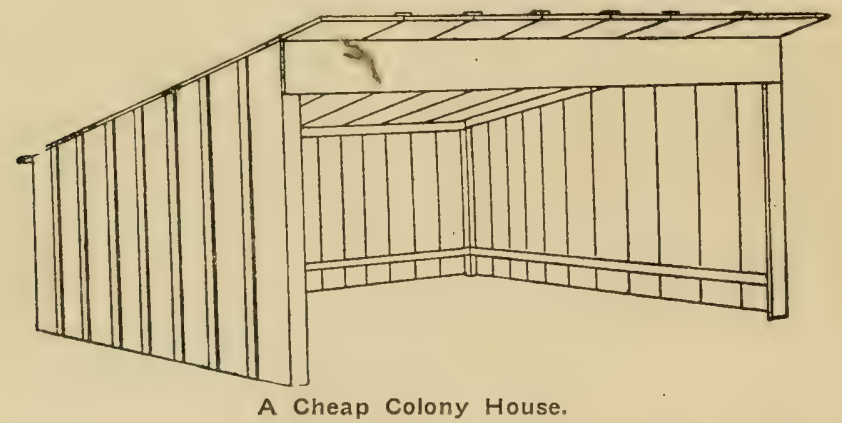

be used as a scratching shed. The same type of house may be used as already described in this chapter, or the tight houses with bottom ventilation. There will, of course, after this set of houses has been built, be some land left. A part of this must be set aside for the raising of young stock, and it is better that the old breeding stock does not go near. this land. 


\section{THE COLONY PLAN.}

There is nothing like free range to make the growing youngsters develop into strong, well-marked adults. One may with care confine the old stock in very limited quarters the year round, and in cases where a very few birds are raised in a season the young birds may be raised in good shape in small yards, but, as a general proposition, if one is raising any considerable number of chicks they should arrange to give them as large a

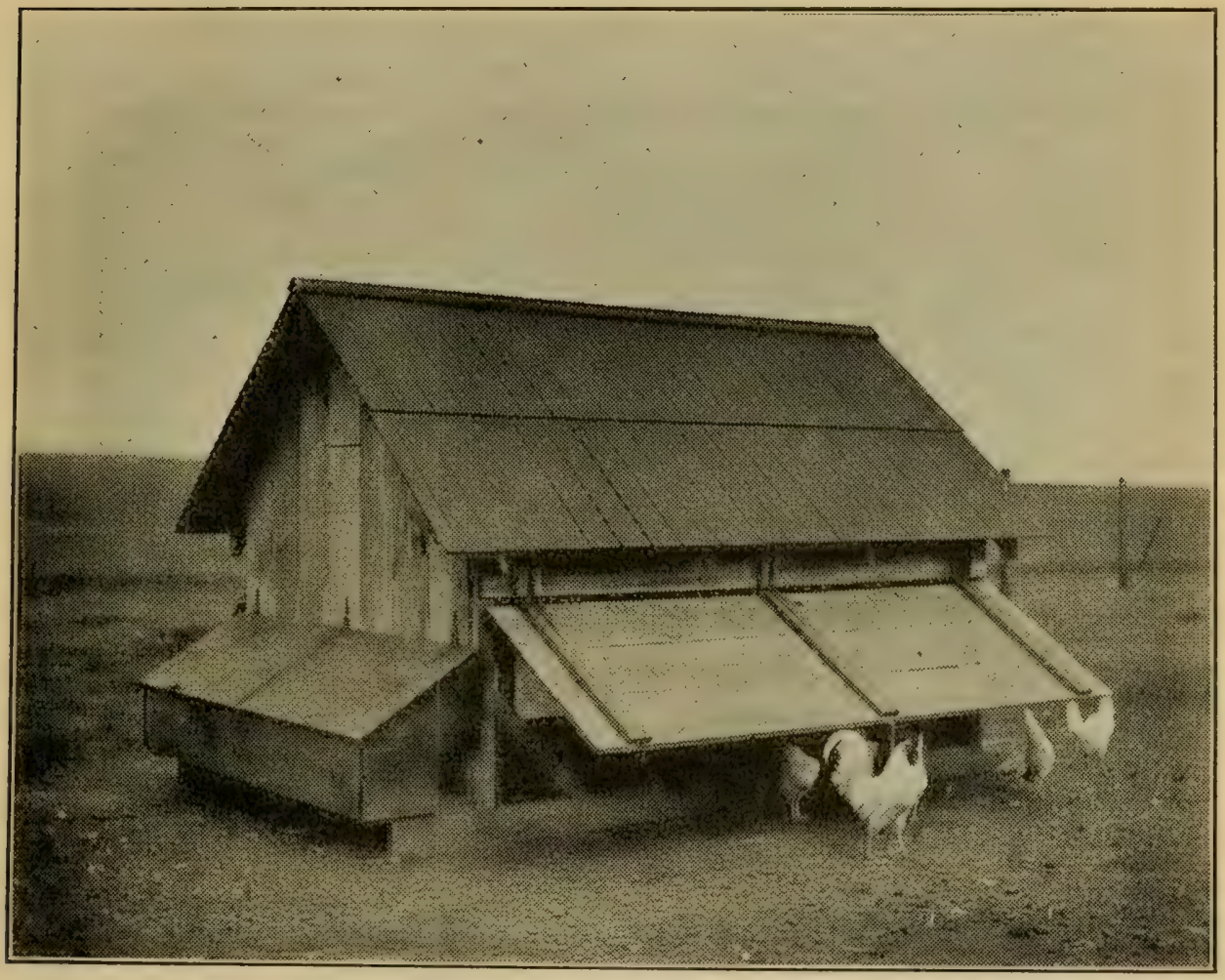

The Rhodes Movable Colony House.

range as their space will permit. In order to do this at the least cost and have the birds in as small flocks as possible at roosting time the best plan is to divide the flock off into, say, lots of 25 to 50 each, selecting those of about the same age and size, and either place these in small colony houses enclosed in large runs that have been planted to some grain crop or scatter them over a general range. Both of these methods have been used by successful breeders. Some breeders use colony houses with eacl colony fenced off in half-acre lots. Others who are using colony 
houses very successfully allow the birds to range at will over a given range during the day, but require them to roost in small flocks at night. It is sometimes necessary to place a temporary fence around the colony house for a few days after removing the birds to the new quarters, so that they can become accustomed to roosting in the colony houses; after that they will return to their proper houses, no matter how far away they may range during the day. In feeding colony birds it is best to scatter their grain feed over the range as much as possible, and make them hunt for it. The dry feeding method which is described in another chapter is a very easy method of feeding birds on a range.

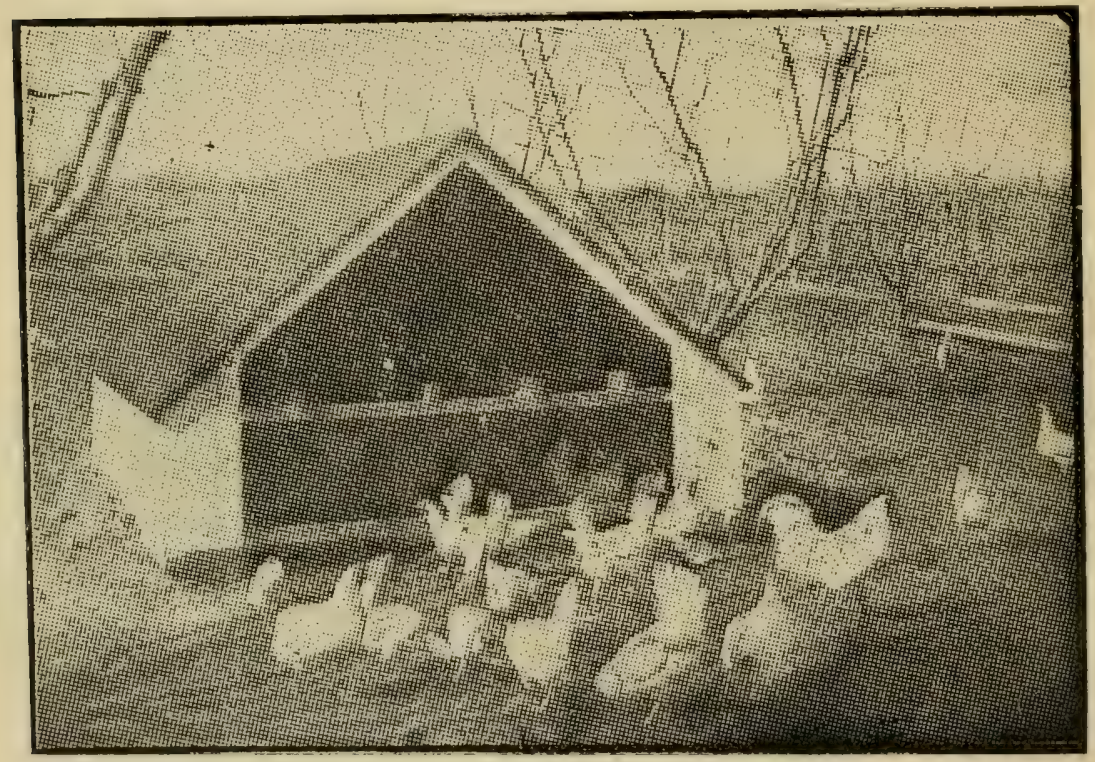

Biddie's Bedroom, Richland Egg Ranch Colony House.

We give herewith cuts of several good colony houses. The mushroom house can be built any size desired, but a house 3 feet wide and 6 feet long is considered the best. They set upon legs 16 to 18 inches off the ground; there is no floor in these houses, and the air can circulate freely beneath them; the sides are 36 inches high, covered in tight with a good roofing paper. The roof is made separate from the house proper, and can be removed each day to allow the sun to shine in on the roosts, and allow the attendant to spray the inside walls; this is also covered with roofing paper. The whole house is made of light lumber, and can be easily moved from place to place. The illustration 
of "Biddie's Bedroom," as it is called by one of our foremost California colony house adherents, is described by him as follows:

"To start with, I will say that I have used this style of roosting house for over ten years, and I am as well pleased with them now as ever, and the only change that $I$ have made that does not show in the cut is to saw out the two feet of $2 \times 3$ between the two center roosts so that a person can walk into the house and avoid stepping over the roost support. I run braces from the front lower corner so that they come directly under the center roosts, and this gives the roost support all the strength that is needed.

"The house is 8 xio feet, and two feet to the eaves, all the framework, including the runners, is $2 \times 3$, and the walls and ends are IXI2, shiplapped so as to avoid using battens.

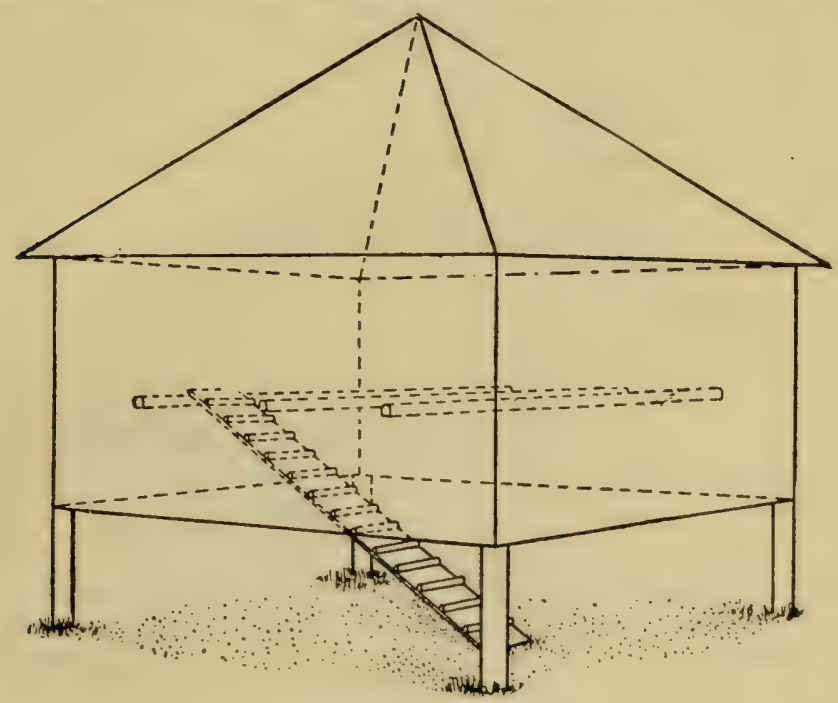

The Mushroom House.

"The rafters are 5 feet 4 inches long, and three pairs are used; $1 \times 6$ is run all around the outside of the roof to form the eaves and also to make it tight; eight pieces of Ix4 are used for sheathing, and the sawed shakes are laid close so that there is no dranght from that source; the only opening is the front, which is open at all times. The opening is faced to the east, as here there is no wind or rain from that direction. The houses do not require cleaning, for they are on runners, and are slid along about fifteen feet each time, so that they are on fresh ground and much cleaner than one could do it in any other manner.

"I have never found a louse in the houses in all the years that they have been in use, and I have never used any form of 
lice killer in them other than whitewash; for appearance only. As to the cost, will say that $\$ 6.50$ will cover it, and seventy-five birds will find all necessary room for sleeping without kicking the covers off or falling out of bed."

\section{HOUSING THE YOUNGSTERS.}

One of the first stumbling blocks encountered by the new breeder is the overcrowding mistake. A poultry raiser will hatch a large number of fine, strong chicks and carry them along to the weaning period in fine shape, when all at once they will notice that the chicks that were doing finely, growing like weeds and feathering in fine style, commence to droop and lose their vitality. This can almost always be traced to one of two causes, overcrowding or lice, and frequently both. Those who use brooders generally have their youngsters at weaning time in flocks of from IOO to 200 chicks, and instead of separating them into small flocks, frequently make the mistake of placing the whole lat in one roosting house. It is not so necessary that the

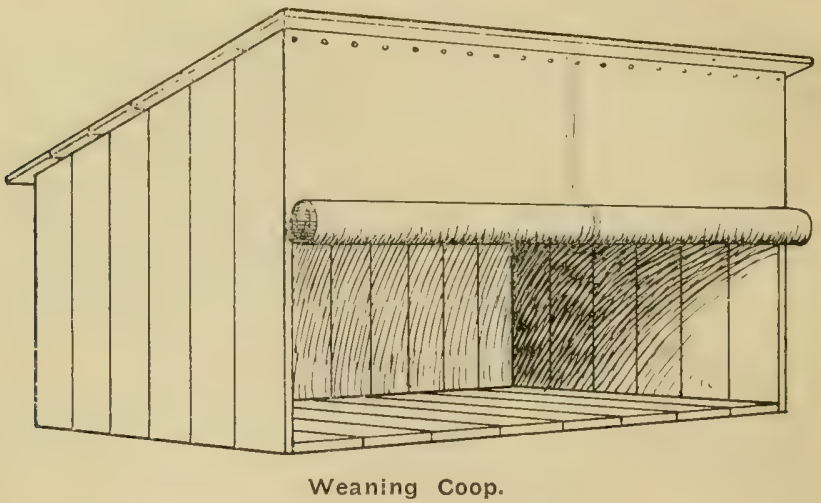

young birds should have different runs, but it is absolutely necessary to have them roost in small flocks. We have found that 25 do better than 50, and we believe it pays, and pays well, to keep the roosting flocks down to not more than 25 birds in each house. Much of the trouble from colds is caused from chicks overcrowding. In a large flock a chick is one minute too cold, and when he crowds his way into the flock becomes too hot, and the bird he crowds out becomes cold, and consequently contracts a cold, and later roup. Then many of the weaker chicks in a flock are actually crushed and injured inwardly. Much foreign color is also brought about by crowding, and it has been the means of spoiling many an otherwise choice show specimen. When the new adult feathers are in what is known as the bloom, the overheating and cooling of a bird and the injuring of the 
new feather frequently makes it come in with foreign pigment showing, when had the bird been housed properly the feather would have come in with the right coloring. In view of these facts it will be seen that crowding should be prevented if one wants to raise a flock of strong, well-marked birds. The best method to keep youngsters from huddling in one corner of their house is to get them on the roost as soon as their size and feathering will permit. A few crooked breast bones will result from teaching the chicks to roost early, but the health and vitality of the general flock will more than make up for. the few that may contract this very common defect. The cut of the cold brooder shown on this page has proven very satisfactory with us as a means of getting the weaned chicks to roost early.

This little house is $3 \times 6$ feet, 3 feet high in front, 2 feet in rear, and can be built of any cheap lumber, such as old drygoods boxes, shakes, etc. The house should have a good, tight floor, on which could be laid a framework covering the entire inside

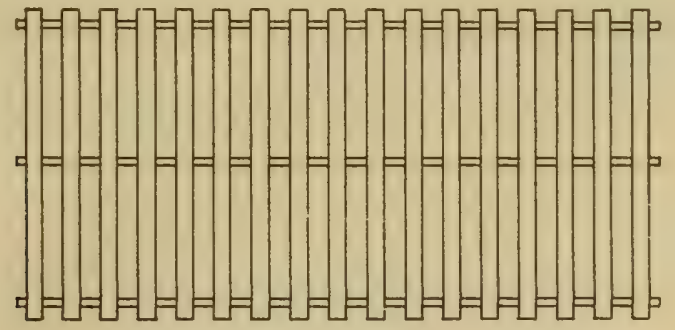

The First Roost.

floor space, made by laying three $\mathrm{Ix} 3$ pieces on edge, and covering with common laths, 2 inches apart. These answer as roosts, and at the same time allow the birds to get quite close together, while the spaces between the laths prevent them from crowding or huddling in the corner. In front of this shed coop a burlap curtain should be hung, and after the chicks are all in at night this should be let down in front of the coop. If possible, it is better to place these cold brooders in a protected place, such as a shed house enclosed on three sides, but where this is impossible they can be placed about the yard as any other poultry house. In a coop the size of the one described above, fifty youngsters can be housed until they reach the fryer size, or until they are, say, three months old. We know of no better method of teaching the birds to roost, and at the same time keeping them from crowding, than by the use of this little coop. Of course these houses should be kept well cleaned, and the roosting frames made in such a manner that they can be easily removed from the coop, allowing the attendant to thoroughly clean the coop, and at the same time sun the roost and treat them for lice and mites. 
THE TRAP NEST.

By the use of a trap nest we soon learn the good and bad qualities of our hens and their general condition. We also learn that the careful use of a practically constructed trap nest should be the means of building up a profitable laying strain. The trap nest illustrated on this page has been used successfully by many California breeders. It is described as follows:

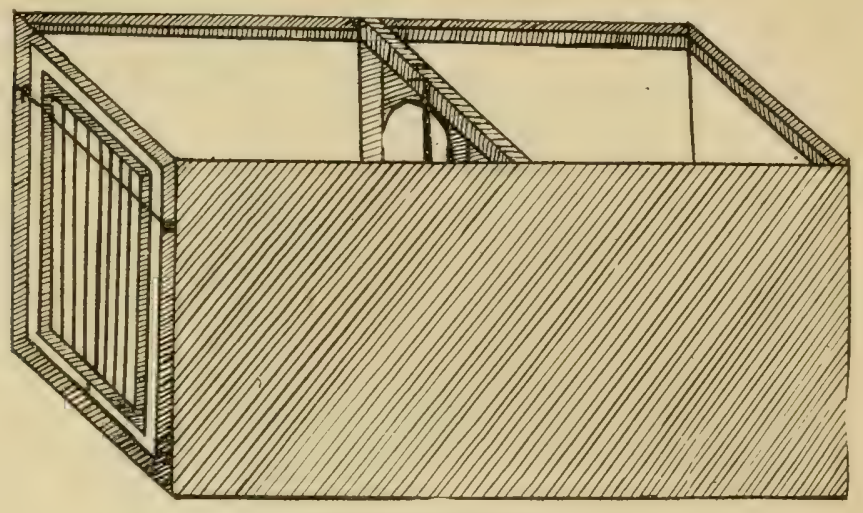

Trap Nest-Figure 1.

Figure I is the box or nest without the top. The top is made of boards large enough to make a good snug cover. The size of the whole box is I4XI4, 28 inches long. For the door I make a frame and cover it with wire; I used straight wire, but

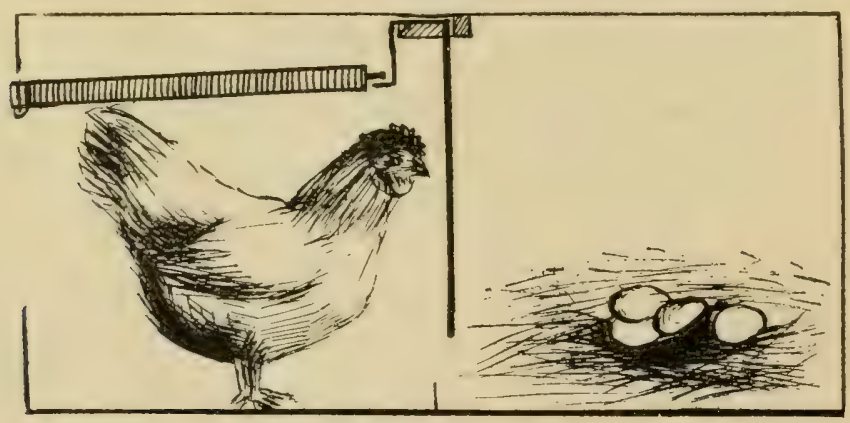

Trap Nest-Figure 2.

one can use wire netting if they choose. For the hinge $I$ use a piece of heavy wire which I nail firmly to the door and let it rest in small staples at the end of the box. Note figure $I$. The trigger is made by having a wire to run in front of the hole in the partition, so that when the hen goes from the front to the nest she pushes it asicle, which lets the door fall. Note figure 3 . Figure 2 shows the hen going into the nest. You note that a nail 
has been driven in the door. This nail rests on the wire that comes in front of the partition. This wire is bent over a IX4-in. board that goes alongside of the partition, see figure 3. Figure 4 shows the nest as one looks from the top, showing the gate raised and trigger set.

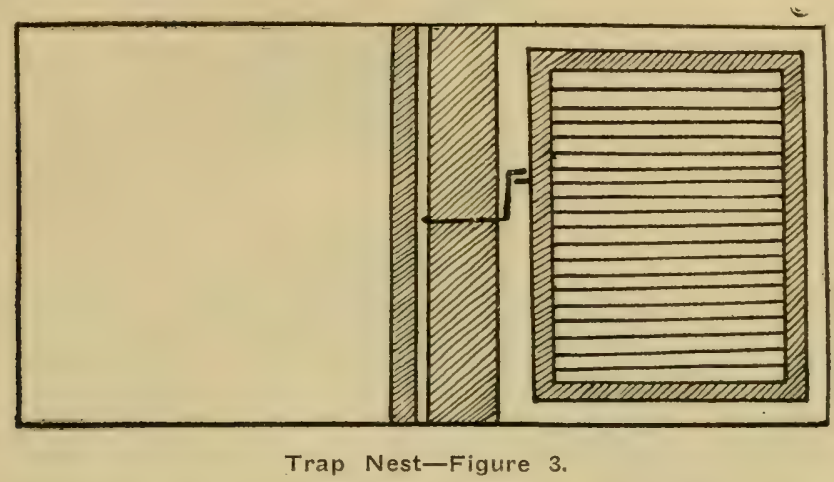

When the hen goes into the nest, the door drops behind her and she has to stay in the nest until you release her. You release the hen by lifting up the cover of the box; if your hens are banded you can have a record book and keep track of each hen

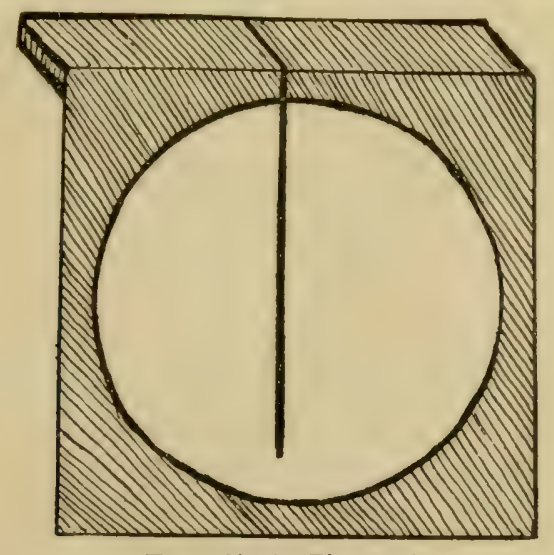

Trap Nest-Figure 4.

by marking the number of the leg band on the egg, and by putting the band number in a book you can credit the hen with each egg as she lays it.

\section{MARKING THE CHICKS.}

Those who have been rearing high-class fowls any great length of time have already learned how to mark their chicks so as to distinguish them when matured, but among our readers there 
are undoubtedly a number who will be glad to be instructed in this matter. Marking the chicks has a number of advantages. If we are going to breed intelligently it is absolutely necessary that the chicks be marked, the progeny of each mating receiving

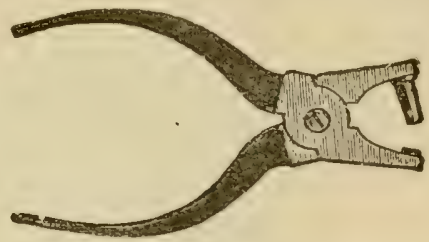

Punch for marking Chicks.

a distinctive marking. In mating up the pens another season this will be a safeguard against in-breeding, as it is possible by this method to know just what relation (if any) the fowls bear to one another. Again, by marking the chicks from the different matings we can know which mating produced the best chicks and can remate the same birds. In buying eggs from another breeder, it is always desirable to know what kind of mature fowls the eggs produced, and it is impossible to know this unless the chicks are marked so that they can be distinguished from other fowls on the place. Besides, we can always tell the age of our chicks if they are marked and a record is kept of the hatches.

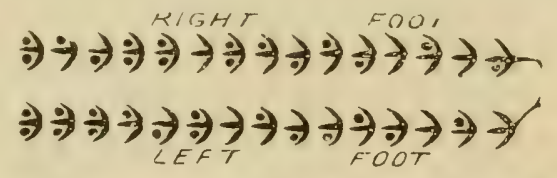

Diagram showing Some of the "Web Punch" Marks.

The diagram herewith shows how the chicks can be marked in fifteen different ways. The two rows of figures represent the right and left feet of as many chicks. The holes are punched in the web of the feet by a punch made especially for this purpose. It is a practically painless operation and the chicks do not mind it much. Be careful not to get the outer edge of the web or it will tear out. As each brood is hatched carefully mark each chick, using a different arrangement for each lot of chicks-that is, marking all chicks from one pen one way, and from another pen another way, changing the arrangement in a manner similar to that indicated by the dots in the diagram just as they appear in the web of the chicks' feet. Opposite this diagram record the pen or breeder from which or whom the eggs were secured, the date of the hatch and any other information that will be of use to you in the future. The above diagram shows only a few of the many ways a chick may be marked in the webs of the feet. 


\section{A SAFE BROOD COOP.}

Herewith is presented an illustration of a chicken coop which we have taken much comfort in during two seasons. We used boxes $18 \times 18$ inches by 3 feet, building the runs of such scraps as are to be found around a place and that can be manipulated by a woman with a saw and hatchet. The front of the box is boarded up about two-thirds of the way across, the other third being left open for the hen and her chicks to pass in and out. The closed end of the box furnishes a snug, warm corner during the night and bad weather. Over the yard is tacked, first, wire

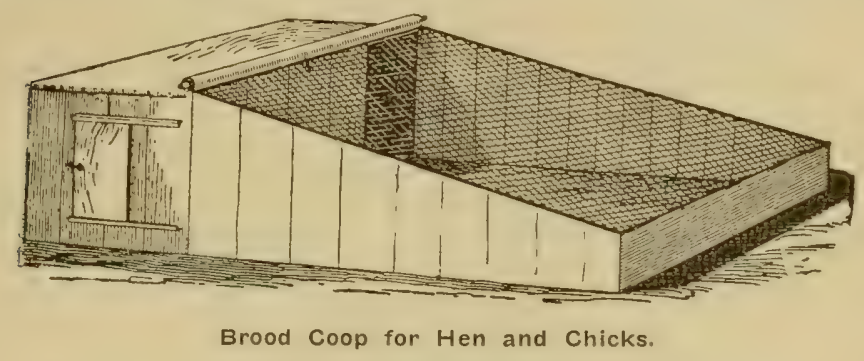

netting, I-inch mesh, so that rats, cats and other prowlers cannot get in. A strip of common table oilcloth is nailed over the top of the box and hangs down behind the box far enough to preent the rain from leaking in. The oilcloth is held in place by laths being nailed along the edges. Another strip of oilcloth, large enough to cover the yard, is rolled upon a stick, one end of it being fastened to the top of the coop on the front end of the box. During stormy weather this can be unrolled downward so that the oilcloth covers the wire netting and thus keeps the ground in the yards dry. After the storm is over this oilcloth is rolled up, as rolling it up prevents it from sticking or cracking or being otherwise ruined as when left lying around loose. In the end of the box a slide door is placed.

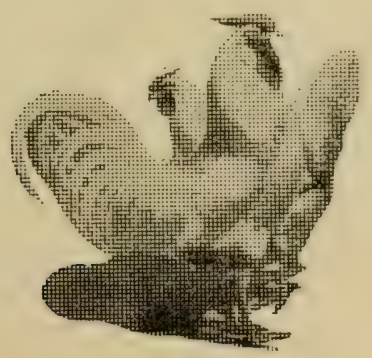




\section{Care of the Breeding Stock.}

Every poultryman must be a student, especially in the way of feeding; no set rules hold good. Fowls at different times need different amounts of food, while the various breeds also differ as to the amount of food they consume. A hen when in good laying condition, needs more feed than when poorly laying. or not laying at all.

The ration given in the chapter on "Care of Birds during Moult" is a fine one for laying hens.

Fowls that are crowded do not respond with a profit. It may pay to have a small flock, and yet the addition of a few more birds may change the profit to loss. Sometimes more fowls can be kept together in the poultry house in the winter season than in the summer, and, in fact, on cold nights it may be an advantage

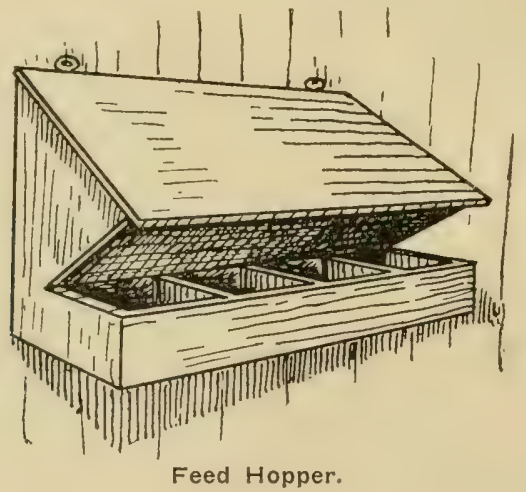

to have enough of them together so that the animal heat of their bodies may raise the temperature of the house; yet they should not be crowded or they will not thrive. During the warm season each hen should have at least ten square feet of room, but in winter five square feet may be sufficient-that is, a house IOXIO feet should accommodate ten fowls in the summer, but in the winter season such a house can be used to keep twenty hens with less inconvenience than to put ten of them together in summer. Many of the breeding birds when fed the concentrated rations necessary for large egg production grow fat, and a fat hen will rarely lay a strongly fertile egg. All hard grains should be fed in deep litter, and it is far better to give the bircls all the possible range. I see many birds kept in small runs with a big 
lot next to them fairly calling to the birds to come and enjoy its sweet young growth. Let one pen out for a few hours at a time if they cannot all have free range, and see the difference in the hatching of the chicks. The breeding birds must be given all the ingredients for a perfect egg. How can one expect a lively chick from a badly nourished hen? Firstly and lastly, give the birds all the cool, clean water they can drink. Think how much of the egg is composed of water, and furnish all the biddies want. In wet countries north and east the birds can scratch bugs and worms enough out of the ground to furnish all they need of animal matter, but in our southern land we must supply them at all times with some artificial animal food. Many use a beef broth as the basis of the morning mash ; others feed dry beef scrap in hoppers; others furnish the necessary amount in green bone;

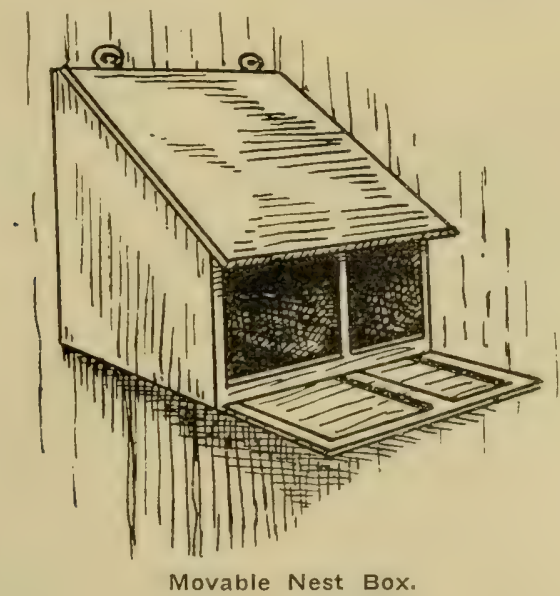

but, whatever your system is, see to it that they get it in some form, and do not grumble when the egg basket is light and lay it to the breed, if you neglect feeding the proper amount of animal food. I have found that with all the green food they can eat, the grain bills reduce fully one-half. In the winter season I feed the young wild clover, the baby barley, wheat, etc., and in the summer time I feed soaked alfalfa clippings. Green food is one-half the ration of a healthy laying female. No liver trouble, digestive disorders or colds will come if the blood is kept in condition by plenty of green food. / Please do not forget the grit and shell. I would rather order the tailor to make a frock without thread than ask a hen to make eggs without shell, and digest her food without grit. Be sure that it is fairly large and sharp. And first, last and all the time, keep up the crusade against lice. Spray the houses every month in winter and every week in summer with distillate and carbolic. Paint the roost with 
one of the good lice-killers just before roosting time, and then if you have a suspicion, after furnishing the proper dusting places, that your birds are not perfectly comfortable, take them one by one and powder them well, or put them in a tight box painted with lice-killer, with only room for the head to come out. Many people do not realize that the males need a tremendous amount of attention for this pest. If they have any lice they will transmit them to the females, and the male is less able to rid himself than the female. They seldom dust, and should be powdered well all during the breeding season; or, better still, placed in the "lice box," which should be a part of every properly appointed breeding pen. The nest boxes should be cleaned often, and either

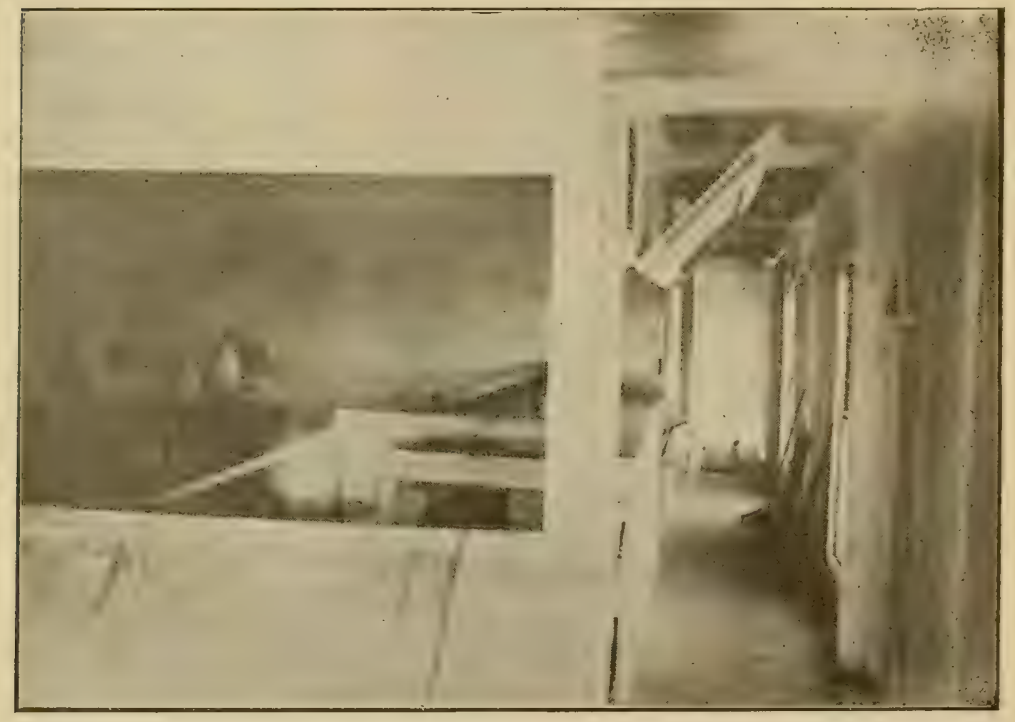

Interior View of Connected Poultry House System Showing Alley and Method of Cleaning.

powder or sulphur and lime sifted in the straw. The nest boxes should not be painted with strong disinfectants; that would be dangerous to the delicate germ in the egg, and plenty of clean straw and an occasional whitewashing will keep it free from lice or mites. If a breeder would only spend more time watching the birds each day he could tell easily how to care for them. Theory will never take the place of practical experience, and the successful man or woman in any branch of work is the one who is not afraid of work.

Some of my correspondents seem to feel that powdering each fowl is a great deal of trouble. The liquid lice paints for the under side of the roosts just before bed time will often solve this 
problem, or one can purchase a lice powdering machine for a few dollars that certainly scatters the powder through the feathers at a great rate. I can powder a dozen big chickens this way, but they get a deal of the powder in their poor eyes. The birds should always be powdered in a barrel or over a paper, and the powder can be used time and again. I find a good home-made powder is one part air-slacked lime, one flowers of sulphur and one dry road dust. This makes a most excellent and cheap powder. I do not recommend dipping fowls. There is too much danger of injuring the egg organs by over handling, and then, too, the birds are liable to colds. I have never had any trouble keeping down this pest by keeping a good deep dusting place, spraying the houses with a mixture of two gallons of distillate and one teacup of crude carbolic, and keeping the nest boxes clean.

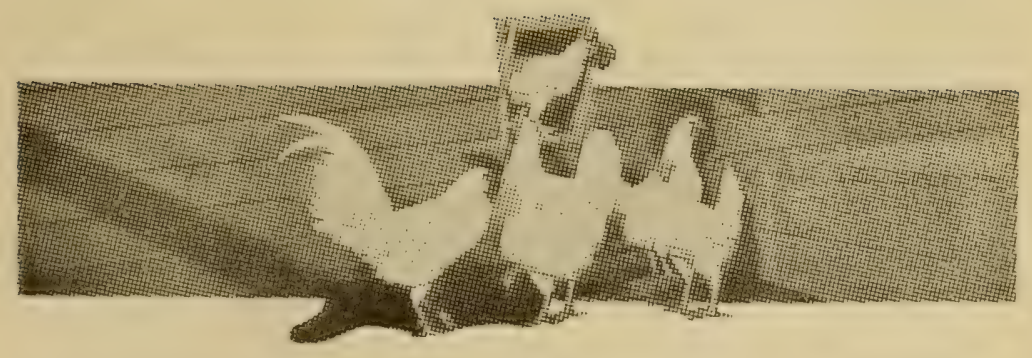




\section{Incubation In California.}

\section{THE ARTIFICIAL METHOD.}

It takes a lot of big wor ${ }^{1}$ s and long phrases to write up an up-to-date article on incubators, but somehow or other we have been reasonably successful with just common United States language when it comes to the hatching question.

Let us be plain and call things by their right names. A fertile egg is a seed, it must have the necessary warmth to sprout and grow, a temperature of $\mathrm{IO}_{3}$; it also must be cultivated, the eggs must be turned, aired and manipulated after nature's ways.

A temperature of 103 seems to be standard, although very few recognize that placing the thermometer differently would necessarily mean that it should read differently, but such is the case. With the thermometer between the eggs showing IO3, the same thermometer lying on the eggs would show I04, or if suspended above the eggs near the tank, it would show still more. We prefer placing the thermometer on the eggs, thus showing the combined heat of the eggs and the heat applied to them, still more I prefer a variation of temperature as the hatch progresses, for instance, IO 2 and IO 3 first week, and IO3 second week, and IO4 third week; this variation being due to the increased animal heat in the eggs as they develop. This temperature will bring good results.

There are bushels of iron-clad rules about turning the eggs just so, but my advice is to turn them as often as you have time and air them as often as you have time. I don't mean that it would be necessary to put in your whole time or even a tenth of it turning and airing the eggs, but I do mean that they should be turned at least once every day, and twice every other day, and that when looking at the thermometer the tray should be pulled out and let the eggs get a whiff of pure air. Why? you would ask. I will tell your. Turning the egg ripens it clear around, the germ comes to the top; every movement of the egg makes a corresponding movement of the embryo chick; besides ripening the shell clear around, these movements wake the chick up, it is exercise for it, it expands, moves, and develops its own strength; this principle applies to all life, whether stock or eggs; for instance a stock breeder would not put up a dam in close quarters to bring a strong young. There is simply nothing mysterious or unaccountable in hatching eggs, a little reasoning in advance of the real thing explains most everything about it. 
Now about moisture! An egg must get rid of a lot of moisture before it can hatch. Eggs during incubation get smaller and lighter; this being the case, applied moisture could not enter the egg. The moisture question and ventilation question are so entwined with each other that the effects of one are often taken for the effects of the other. Personally we do not believe in dosing out the aid in prescribed quantities-there is lots said about governing the size of the air cell by opening and closing the dampers, but we prefer a correct and continuous ventilation. If it is correctly fitted, there need be no dosing it out in a mystical way with dampers. We prefer a constant mild circulation of air and to depend on airing the eggs in open air to conform to nature's ways.

What's the good of airing the eggs? might be asked. In answer will say, that the egg shells, like other things, expand in heat or contract in cold. This expansion and contraction, the result of airing the eggs, breaks down the tough fibers of the shell, and when the chick is due to hatch it can hatch. It also applies to moisture on the same principle that a glass of cold water will sweat in a warm room when the atmosphere is just right. We are sure this moisture, however light, is good for the shells, good for the reason that it is light and affects the shells only.

Good hatches are made with and without moisture, opinions are about equally divided. We are satisfied, in fact, we know it does no harm to apply a little moisture direct to the shells if the ventilation is sufficient. Have tried all the moisture plans and plans without moisture and have, at this time, more faith in the old-fashioned way of sprinkling the eggs occasionally than any new way.

The old-fashioned sprinkling is applied direct to the shells and does not make a continuous mucky air in the egg chamber. It does not matter about exact dates in sprinkling, exact dates would be nonsense, but I can guarantee that you will do the eggs no harm if you sprinkle them with tepid water on the 12th, I 5 th and I8th day.

Do not disturb the trays from this time until the hatch is complete. When all the chicks appear to be pretty well dried, open the machine, remove the trays, and closing the door, except a minute crack for better ventilation, leave the little fellows in the egg chamber for twenty-four to forty-eight hours, gradwally decreasing the temperature until the thermometer registers 95 at the end of twenty-four hours. As the thermometer hangs above the chicks, it is probably a degree less at the chicks' heads. We have found this plan to work very satisfactorily. The chicks go into the brooder accustomed to a lower temperature and ready to eat anything that they can find. We believe 
that most machines do not furnish enough fresh air for the chicks after they are well dried off, so open the door just a little that. they may be supplied.

Many of the new models of incubators have a drawer into which the chick falls and can thus be better tapered off from the 104 of the incubator to the brooder heat.

The temperature of the brooder should be fixed at 90 before the chicks are put in, as its complement of chicks adds two to. five degrees when they are under the hover.

\section{NATURAL INCUBATION.-MANAGING SETTING HENS.}

In preparing to make the spring hatch, we usually take one room of our poultry house, one that has a dirt floor, and take everything ont of it. The interior receives a good whitewash with the spray pump, and lice killer is used until we feel sure the room is free from lice and mites. We take cracker boxes for the nest boxes, and after spraying them in the inside we put in about four inches of fresh dirt; in this we make a nice nest, filling all the corners of the box and seeing that the bottom

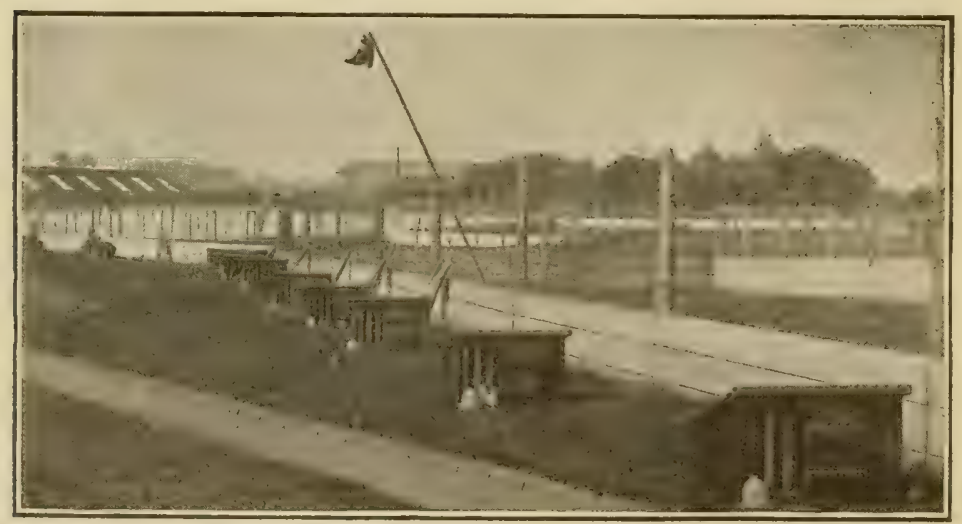

Chickens and Mothers on Alfalfa Range.

is rather flat. Next we take common hay, that is not too coarse, and line the nest, care being taken not to use too much hay, and that the nest is good and roomy. We place these around the wall of the room and have a tow sack ready for each nest. After dark we take the broody hen off of her nest, no matter where she may be (many times we purchase them), and take her to the sitting room. We give her one or two egses, put her on the nest and pull the sack down so she can't get out. Late in the afternoon or just before sundown we go out and quietly draw the sack off. Corn and wheat are set before her, with 
plenty of water and grit, and a place to take a dust bath. If she goes back on the nest we know she is ready for business, and that night we give her the eggs. If she does not go back on the nest, we put her back and try her again, and if she won't work, we take her back where she came from. We usually have from six to ten in one room, and with proper care and attention have very little trouble with them. We dust them about three times with insect powder, taking care not to dust a hen just before she hatches. When setting a hen we give the number of the pen from which the eggs are taken, stating the breed, giving uate for hatching, putting this on a card and placing it in the box where it can be seen. Then we can tell just when to be ready and to look for the young peepers. Regularity in attending will aid onderfully. With feed and water go in the morning and see that their wants are supplied. Always notice to see that no broken eggs are in the nest, as this may cause some trouble. Just about dark take a stroll through the poultry house and see that all the hens are on their nests. We usually do the dusting after night, as less confusion will follow and they will bear handling much better. Set your hens in pairs ar several at a time, and when they come off it's best to have all in the room hatch at the same time for several reasons. The first is nothing so interests a hen as to hear a peeping chicken, and if she hears the noise in her nest she stays with it the more closely, but if it's in the next nest she may jump off to investigate. Give them careful attention when hatching. The next advantage in having several come off at one time is that you can give the chickens to part of the hens, and reset the others. With a good brood coop a medium-sized hen-and they are the best-will take care of 18 to 20 chicks in fine shape, so that you may be able to reset at least one-half of them. Care should be taken that you do not make a hen set more than two terms. Watch out for mites, when the hens want to leave the nest and will jump off quite often, look for mites again. They are the setting hen's very worst enemy. 


\section{Care of Chicks.}

\section{FEEDING THE LITTLE CHICKS.}

So many different methods of feeding brooder chicks are advised by people whose experience and success entitle them to consideration, that what one man or woman says should be taken merely as an opinion until one tries it and finds it satisfactory for his own use. The prepared chick feeds now on the market offer a well-balanced ration of dry grains with the proper allowance of beef scrap, grit, charcoal, etc., in a convenient form and at a reasonable price.

We believe that chicks often get too much food when they are first placed in the brooder, and that heavy losses frequently occur from that cause alone. The best results have been obtained when the chicks were left in the incubator for a day and a half without food after the hatch has been completed, and when placed in the brooder fed one light feed the first day, two

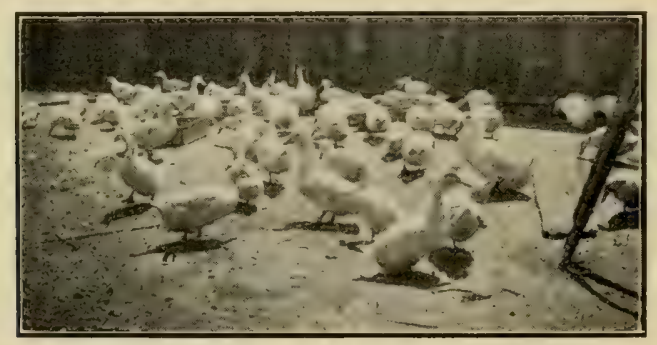

Pen of Young California Pekin Ducks.

the second and three the third. Beginning on the fourth day, five feeds may be fed daily, but they should be very light and never more than enough to satisfy the chicks for the time. A little lettuce or finely-chopped clover, grass, or alfalfa can be fed daily after the first week, and occasionally a change to chopped onion will be relished and prove of medicinal value. For chicks not intended to be kept for breeding a little raw beef is good, but it forces the bird too rapidly to be good for breeding stock.

\section{EXERCISE NECESSARY FOR HEALTH.}

Every inducement should be offered the youngsters to scratch and dig, and the exercising apartment in the brooder 
and the adjacent pen in the house should be thickly carpeted with some good scratching material to that end. Hay chaff is one of the best things for this purpose and can be easily obtained. Anyone who stores or feeds hay has more or less of it and is usually glad to get rid of it. It contains many small seeds and bits of clover, which the little fellows make good use of and for which they will search diligently if healthy and not too well supplied by the attendant. The temperature of the brooder house should not be too high. Some will deny that it is well to heat the house at all, and many, either from preference or lack of equipment, supply no heat to the house except that which escapes from the brooders. When the chicks are small, however, I believe that a moderate degree of heat, about 60 degrees, is desirable in cold weather in that it enables the chicks to spend more time on the floor of the pen getting up their muscle. It should be remembered that 60 degrees recorded when the

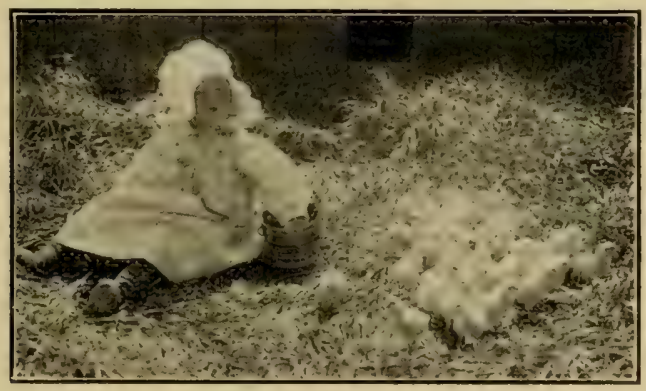

California Productions.

thermometer is three feet above the floor is not 60 degrees down: where the chicks are. Place the thermometer within a few inchs of the floor.

The best manner of feeding is the one that will best promote exercise. If dry food in the form of grain is given it is best, especially in cold weather, when the little birds cannot get outdoors to exercise, to mix this in the litter and compel them to scratch it out.

\section{PURE AIR OF IIIRST IMPORTANCE.}

In rearing chicks pure air is a very important factor that seldom. gets due recognition. Cheap as it is, and necessary for the vital processes concerned in maintaining and developing all forms of energy, it is not unusual to find brooder houses with no provision for anywhere near an adequate supply. Brooder stoves and heaters are burning out what little oxygen there is, and owners and managers wildly endeavoring to figure out some intricately balanced ration to reduce the frightful mortality. Provide plenty of good air under the hovers and wherever else- 
there is a chick, and the science of feeding will be wonderfully simplified.

Pure, fresh water should be always accessible if a dry grain ration is fed. Opinions differ as to the advisability of supplying water when feeding a mash ration, and some poultry men seem to have greater success when giving water and others when withholding it.

The degree of heat will have been gradually reduced to 80 at the end of four weeks, and may be further reduced to 70 at the end of six weeks, where it may remain so long as the chicks need a hover. No more heat is needed in the house than is necessary to remove the chill in the air in cold weather.

A gradual change from the baby food of the first four weeks to the more substantial diet of mash, cracked corn and wheat should be accomplished in ten days or two weeks. This is a period of growth, and the chicks should have all the food they can make use of. More fresh air, exercise, and as much outside run as possible are potent factors in their development. Green food must be furnished daily if the birds cannot have green range.

The growth of chicks is almost unbelievable when fed a simple mash of two-thirds wheat bran, one-third corn meal, with ten per cent. of beef scrap added, three times a day-morning, noon and night-with an allowance of wheat at mid-forenoon and of cracked corn at mid-afternoon. Grit and charcoal should be kept in the pen, preferably in a hopper where it will be clean.

No more food should be given at any time than will be consumed at once, and if any mash is left, it should be taken up when the attendant takes up and cleans the troughs or boards, as he ought to do after each meal.

It is understood, of course, that the grain is fed in litter on the floor of the pen, and of sufficient quantity to induce vigorous scratching, but no more.

\section{AFTER THE EIGH'TH WEER.}

From the eighth week forward different treatment must be accorded those intended for stock purposes or large roasters and those intended for broilers, only the broilers-to-be should remain longer in the brooder house. The others should be placed out in the field in roosting coops if the weather is warm or housed in warm quarters. Occasionally broiler chicks may advantageously be placed outside, especially if errors or carelessness in feeding have noticeably reduced their vitality, or if it is impossible to maintain a temperate heat in the brooder house. Usually it is best to keep them in the house where they will take but a moderate exercise, and will lay on flesh and fat without the toughening of the muscles which takes place when they have free range in the fields. 
From the eighth week to killing time plenty of green food should be supplied every morning; the grain should be fed as before and the mash materially strengthened. Three parts corn meal, one part wheat bran and one part first-quality beef scraps makes a simple and effective fattening food, which, if fed to chicks in good health, supplemented by green stuff, fresh water, grit and charcoal, as directed, will make a full-fleshed, fat broiler of unbeaten quality. One of the most important points to remember is that no mash nor troughs must be allowed in the pens except during the few minutes when the chicks are eating. No other than freshly mixed mash should be fed, and any that remains when the troughs are removed should be taken away and may be fed to old birds.

A potent cause of trouble is overheating in the hovers. When the older chicks, from eight weeks forward, are allowed hovers they will frequently crowd into them at evening, cause a high temperature and lose in the night all the flesh they have gained in the daytime. If they have access to hovers sufficient ventilation must be provided to keep down the heat. Always look through the brooders before retiring and arrange for the comfort of the occupants during the night. Much can be done to that end after the chicks have settled down.

For the chicks intended for roasters no change in composition of the ration need be made except that a larger proportion of hard grain and less of mash should be fed, and the number of meals reduced to three per day. Mash may be fed at morning and noon, or only in the morning, as best suits the judgment and convenience of the feeder, the remaining feeds being of grain, principally corn, wheat and oats.

When it becomes necessary to fatten roasting chickens, they may be confined in yards of moderate area and fed the same as adrised for fattening broilers. Occasionally it may be advisable to place some of the quarrelsome males in a room which may be darkened except when they are eating.

The hatching and raising of chickens, while requiring constant and painstaking attention, is by no means a difficult proposition or one beyond the ability of the man or woman of average intelligence, and the application of common sense will produce satisfactory and profitable results. 


\section{Care of Birds During the Moult.}

During the moulting season (from July Ist to November Ist) the poultry breeder should give his fowls special attention; in many cases, after heavy laying has stopped, the birds are more or less neglected. The mash at this season should be discontinued, as the animal food contained in most of them keeps up the egg production, and it is necessary that the egg production be stopped, if possible. The older hens of good type, and that are to be kept for breeders, should be allowed to set if they become broody in July or August, as the process of incubation is the very best means of carrying out a successful moult with them. All birds should be carefully dusted and the houses be made lice and mite proof, for the little pests have spoiled many a show bird by ruining the new feather. The Van Dresser method has been tried, and many people speak well of it, but personally we think it takes too much of the vitality of the bird that is to be used as a breeder, and many a fine male has been rendered utterly useless for another year by being forced too quickly to moult. A bird must be reduced in flesh, there is no question about that, but it should not be starved down too suddenly. Another error that is frequently made, all birds are treated alike, whether fat, medium or thin, all are starved for three weeks until the fat hens are in proper condition for bringing out the new feather, and the thin ones are drooping and weak, if not practically ruined. So divide your birds before you start the moult, put each male in as large a single pen as you can spare, put the fat hens in a good, large yard and feed as much green food and as little wheat as you feel will keep up their strength, but reduce flesh, put the hens that are in good condition in another yard, and fced a bit more so they will all start moulting evenly; then when you start feeding your moulting foods the work is only half as hard as if the pens had to be fed separately for condition.

Rations for Moulting Hens.-The food requirements of the moulting hen do not differ materially from those of the laying hen. Some authorities prescribe rations rich in protein and low in fats, as containing in better proportion the elements required for feather production.

We began by using such rations, but soon discovered that our hens moulted better, growing a much better and glossier 
coat of feathers, if fed a ration rich in fats. Experience in this respect has been the same with hens moulting in midsummer, and those moulting in late fall, except that the hens moulting in summer moulted much more quickly and often laid continuously right through the moult. For many years our method of feeding moulting hens has been:

Morning: Mash made of Ioo 1bs. bran, 50 lbs. shorts, 50 lbs. corn meal, Io lbs. beef scraps.

Noon or all-day feed: Wheat, sunflower seed or barley, broadcasted in! scratching pens littered with clean straw.

Evening: Cracked corn scattered in the yards, but all should be worked for so the fat does not accumulate and hurt the bird for laying.

Alfalfa or cabbage before the fowls all the time.

The mash should have a portion of linseed meal a few times a week.

Many of the mills are making a good moulting food, and it can be frequently purchased far cheaper than the small breeder can buy the separate ingredients and mix them. This ration will make a beautiful, glossy feather, and get the bird in good condition in a short time.

The males should receive every attention, and not be mated to the females again until their new feathers are nearly perfect. The breeder of exhibition stock should keep the show birds in shady pens, and it is much better for them not to be mated until the show season is over. To the new breeder this is genrally too much of a deprivation, but the old breeder generally picks out his stock at the beginning of the moult, and it stays on deep straw, in well-shaded pens, and has no broken feathers, nothing, in fact, to count against it in condition. A great many breeders give a tonic of iron during the moult, and it is a most excellent thing for black or buff birds, but is apt to bring too much creaminess out in the white birds. It is a bad idea also to give white birds much yellow meal or yellow whole corn, for the same reason, but in this country we feed so little corn that it is hardly worth making any change in the color of it. While the birds are being fed for feather is a good time to get thoroughly acquainted with them, for a bird made gentle by petting and attention generally scores a few points higher when the judge gets hold of it. We have seen a really magnificent specimen crouch down in the coop and flutter around so wildly that no idea of his carriage and shape could be obtained. Do not forget that the birds are in a more delicate state of health during this time, and house them in draught-proof houses. So many people have no idea of treating a bird humanely, yet are mortally offended if told so. They let the poor things roost 
in the trees or houses so filthy or draughty that a cold is always threatened, and then when a stranger wants to know about the poultry industry they are informed that there is no money in it in California. Give plenty of cool, fresh water, have charcoal, grit and shell before them all the time, and furnish an abundance of green food.

Just remember how good the green salads, fresh fruits and cool drinks taste this season of the year, and do not forget the chick-a-biddies. If you give your birds this attention and care during the heated season you will have early eggs, chicks before your neighbors, who are too negligent to work for the greatest good to their birds, get your cull cockerels sold at top prices, and have a fine lot of early layers.

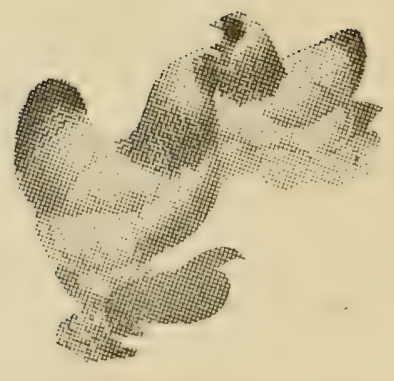




\section{Profitable Egg Ranching.}

A few years ago in California the big egg ranches ran almost entirely to the Leghorns, but with the introduction of some of the newer breeds and some strains of the older ones especially bred to heavy egg production as well as size of bird, there is a change and one frequently sees a flock of a couple of thousand of one of the American or English breeds on an egg ranch. We believe, however, this is the exception and most of the market eggs in California are from Leghorn stock.

Two plans of egg ranching are followed; the extensive, or colony, and intensive, or yarded, flocks. The extensive and intensive plans both have their advantages. The colony idea is one which demands less care, and those who follow it say they have as good results as the man who has small yards and pens for his flocks. This controversy proves but one thing conclusively, and that is that either plan will be successful if followed with persistence. The colony plan demands larger farms and gives the poultry farmer less insight into the workings of the individuals of his flocks. The attention is less than that demanded by the small pen plan, and the food supply is smaller. To offset these advantages the advocate of the intensive plan says he gets better results, knows what each hen is doing and can thus cull out the poor layers, and gets more eggs at the time of the year when eggs are highest in price.

Under the colony plan the poultry is housed in small movable buildings, which are scattered over a large field. The estimate is that there should be two hundred hens to the acre. If a man desires to raise two thousand hens he must have at least ten acres for them in addition to the necessary ground for his own house, barns and outbuildings. This ten acres, however, can be planted to orchard, and both hens and orchard are the better for it. This plan gives the chickens plenty of exercise, as they will roam over the entire ten acres, each colony returning to its own house at night. The feeding of these colonies is simplified by having hoppers which are self-feeding, thus obviating the necessity of attention except at stated periods when the hoppers are to be filled. Water is supplied from continuous founts on the same plan. In adclition to this, a feed of mash is usually hauled around to the 


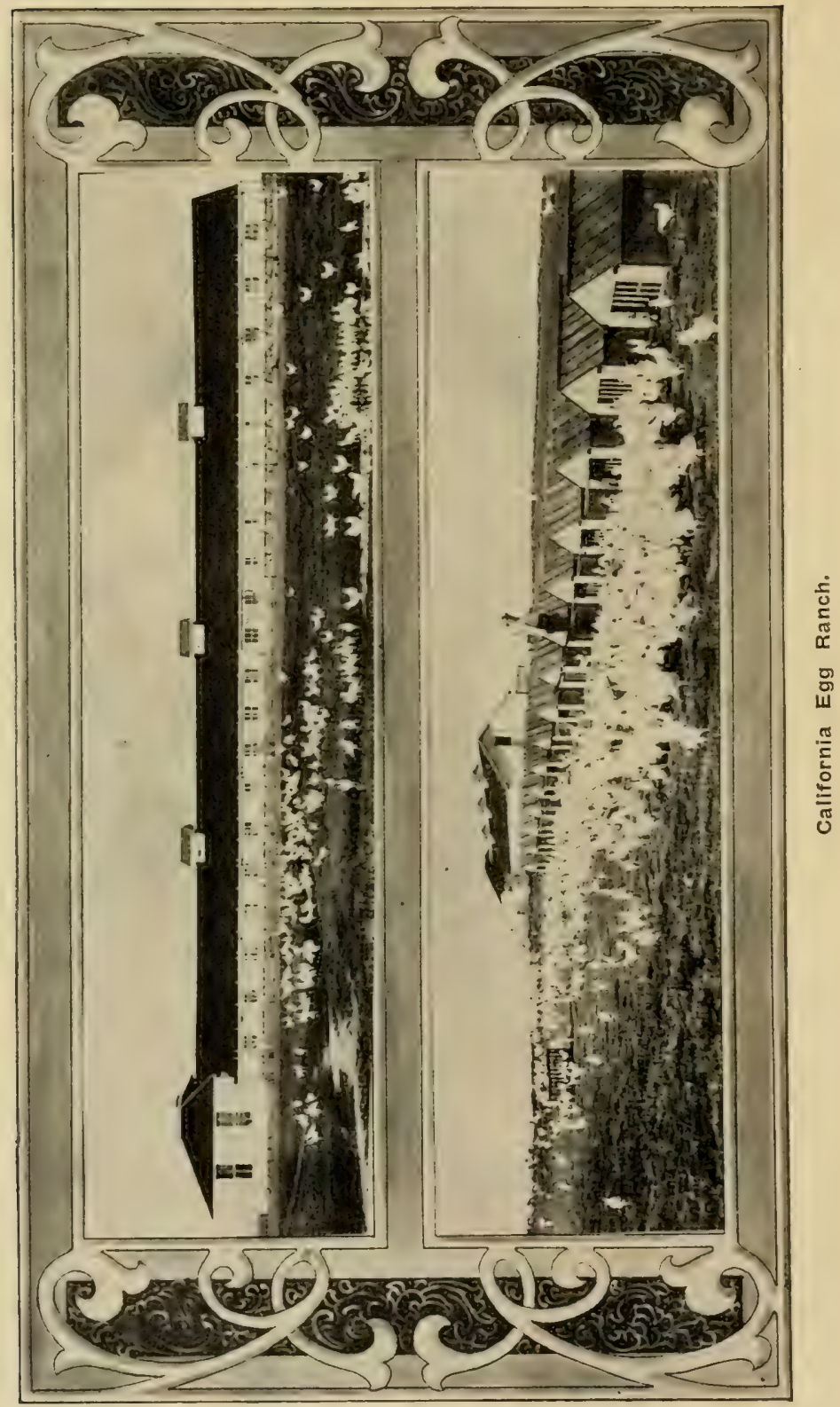


different houses once every three days. This sort of poultry raising minimizes the work, making the egg gathering the most arduous of it all.

We have in our chapter on Poultry Houses some very good colony houses and if the pullet is started laying in one special house the chances are that she will always go home to lay and roost in the same house no matter how close the others are. To our mind the strongest argument against the colony plan is that too much of the birds' physical energy is wasted in ranging, energy which if properly conserved will turn to egg production, but one of the advantages is that the birds need less care.

On the intensive plan two thousand birds can be cared for on five acres of ground, and they will be constantly under the eye of the owner. The grain should be fed in deep scratching material for the reason that with the small yards exercise must be induced to keep the birds from growing overfat. A plant of this style can be laid out very cheaply; any of the houses in "Poultry Houses" chapter can be used and built in a continuous line. One of the best-paying poultry plants that we know of has the yards of two rows of houses running to a single alley thus making the work of feeding, gathering eggs, watering and cleaning much easier. The egg production is better in the intensive plan as the "drones" can be more easily culled out and the food goes more to egg production. This plan is also better when it comes to the care of chicks raised in brooders.

The one object of all poultry keeping is profit, and as in practical egg farming it is the fall and winter eggs that pay the best profit, it is necessary that we study the conditions favorable to getting eggs in fall and winter if we would have the best profit. It is very generally conceded by observing poultrymen that the pullets are the early winter layers, hence it is obvious that we must look to the pullets for the eggs that pay that best profit. It is equally well understood, by experienced egg farmers, that to be good fall and winter layers, these pullets must have been early hatched, and have been kept growing so that they come to full size and laying maturity before winter overtakes them; indeed, the writer has frequently expressed the opinion that the key note to the best profit from poultry can be laid down in these three short rules :

First: Hatch the chicks early.

Second: Keep them growing so that the pullets shall come to laying maturity before rainy weather.

Third: Keep them laying by good care and good food. 
The full story of profitable egg farming is condensed into those three short rules.

It is equally well understood that the chicks hatched from the eggs of pullets are generally slightly smaller, and are likely to be less hardy and vigorous than the chicks from the eggs of year-old hens, and the wisest poultryman hatches the chicks which are to be the future laying-breeding stock from the eggs of year-old hens. This principle of pullets for layers and year-old hens for breeders makes it easy to plan our method of procedure. To get the best all-round results, three-fourths of our stock should be early hatched and well matured pullets, we will get the bulk of the early fall and winter eggs from them-the eggs which pay the creamy profit; and by selecting for the future breeders the best layers among those pullets and breeding from them when they are year-old hens, we will strengthen the laying habit and thus increase and develop it. It is well known that not every pullet hatched from eggs produced by a great layer will be a great layer, just as it is known that not every colt from record trotting ancestry will prove a great trotter; still we will get the best results if we breed from stock of known great-laying ancestry, because by breeding from that stock we will strengthen and develop the egg-producing quality.

A point frequently overlooked in connection with pullets for layers is, that if the hens are all kept over a second, third and fourth year they. occupy the house room and are eating food of pullets which would pay double the profit. We do not claim that a pullet would lay twice as many eggs as a hen, the point we want to bring out is, that a greater proportion of the pullets' eggs are produced at the time when prices are highest and pay the best profit. Eggs are high in price at that time because the hens have not recovered from the moult and are taking a rest, and also because many of the pullets are late hatched and have not begun to lay. If we are so fortunate as to have early hatched and well matured pullets laying at that time we are getting the "cream" of the profit from egg production.

Another point which we need to keep in mind is, that if the hens are kept over we lose the amount which they would sell for, and if we have good stock the amount the hens sell for materially increases the profit account.

Some writers claim that the cost of raising pullets to take the place of the year-old or two-year-old hens must be considerable, but we have proved, year after year, that cockerels hatched with the pullets can be sold at from four fo five months old for enough to pay for all the food eaten 
by both themselves and the pullets, hence, considering the cockerels as simply a by-product, the pullets cost nothing for the food they have eaten up to laying maturity. Some will be inclined to doubt this statement, but, as we stated before, we demonstrated it again and again, and can prove that the pullets at laying maturity cost absolutely nothing but the labor of caring for them; obviously then, all of the money received for the sale of the year or two-year-old hens is clear gain.

It is the old familiar story of the slow shilling and the nimble sixpence; the latter pays its owner many times more interest than the slow shilling. The pullets not only pay us the better profit in the increased number of eggs produced, but the failure to sell the old hens entails the expense of house room and food which could have been used by more profitable pullets, which would be so much better layers; and it also cuts us off from the income of the sale of the hens themselves.

With these several points of advantage clearly in mind we think our readers will understand the importance of the motto- "Pullets for layers, and year-old hens for breeders." 


\section{Poultry Plant for Breeding Stock and Eggs for Hatching.}

In making a start in the thoroughbred poultry business a most essential thing is that we start with good stock by getting the very best that one's money can buy. A beginner who purchases a trio of extra choice birds or a setting of eggs from a high-class breeding pen is getting much more value for his money than one who buys a large flock of cheap birds or several hundred inferior eggs. Most beginners have little idea how much time is saved, and time is money, by buying good stock. Another point, don't breed too many varieties. The whole of a life time is not too long to get the best out of one variety, and the man: who devotes himself to one or two varieties gets the best there is out of them and will achieve the greatest success. The greatest successes among poultry men today, as a rule, are those who have made their reputation with one variety; the men who get the largest prices, who do the largest and best business, are those who are devoting themselves to the breeding of one variety and making that a specialty; who are putting into the development of their chosen variety all the energy and ability with which they are endowed.

In California the poultry plant for fine breeding stock is a big paying proposition, the people of this state and what we consider tributary to it, Arizona, Washington, Oregon, Nevada and Montana in the States, and Australia and Hawaii, being fanciers of the best type. By this I mean the fancier who is always looking to improve his flock and is willing to pay a good price for eggs and birds necessary to accomplish his purpose. We have always received good prices for our own stock on our ranch, eggs ranging from two and a half dollars per setting to twenty dollars, and hens and pullets from five to twenty-five, cocks and cockerels from ten to one hundred and twenty-five. These are not exceptional prices, but prices maintained throughout four years of steady demand. We know of many ranches who book all of their eggs at above prices for the season by the first of December. The rancher who starts out to maintain a flock for the sale of fine eggs and exceptionally fine stock should never for any reason allow a cull to grow to maturity. The birds should 
all be of standard quality and well mated to bring forth offspring as good or better than the parent stock. The breeder should exhibit his stock in the poultry shows of his natural buying territory and by having the right kind of stock can rapidly become known as a fancier from whom to obtain good stock, and by maintaining the highest quality will be able to have a good income with less work than the commercial egg rancher.

\section{HOW AND WHEN TO ADVERTISE.}

Having made a start with good, thoroughbred stock, the next thing is to let the public know it and solicit orders by advertising:

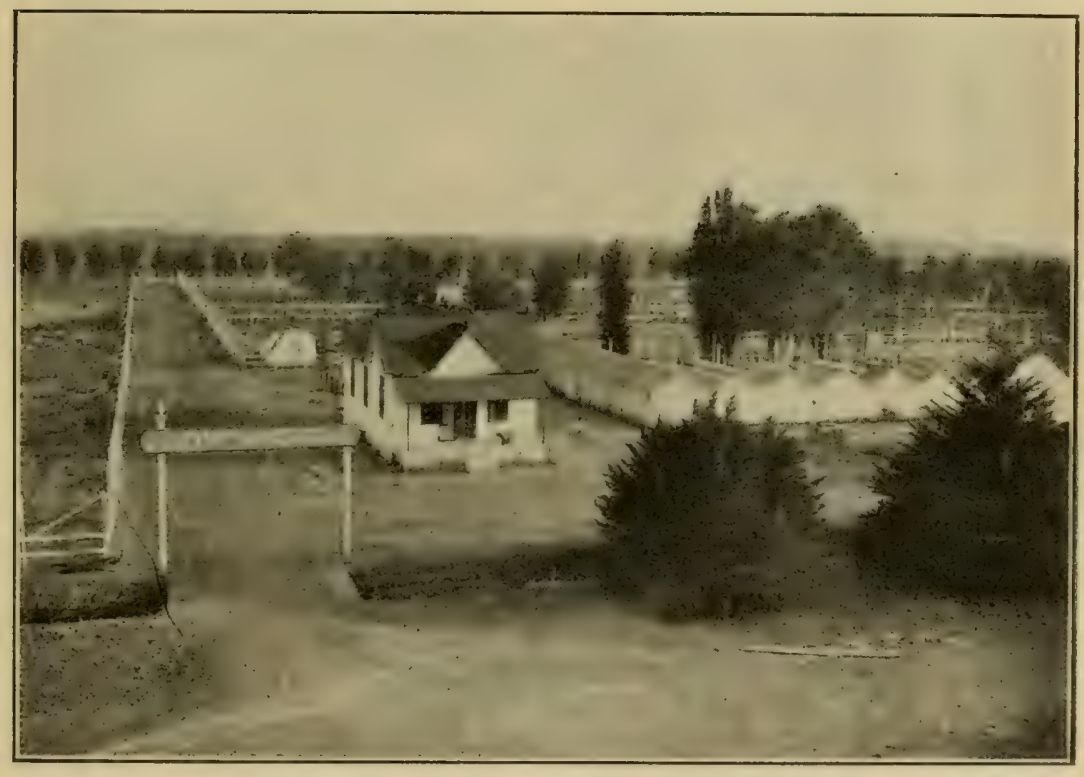

View of Successfil California Poultry Plant.

The merchant or storekeeper tells the public who he is and what he offers for sale by signs over his door and on his windows, and by displaying samples of his wares in his windows or beside his door; that is one way of advertising, and such advertisements are likely to be seen by those passing the store. Another method of telling people who one is and what one has for sale is by publishing an announcement in the advertising columns of the local paper; the card in the local paper is another form of the "sign " or the "window display." For the poultryman who has breeding stock or eggs for hatching for sale the poultry and farm papers are "local." Through them he will reach the public that is in- 


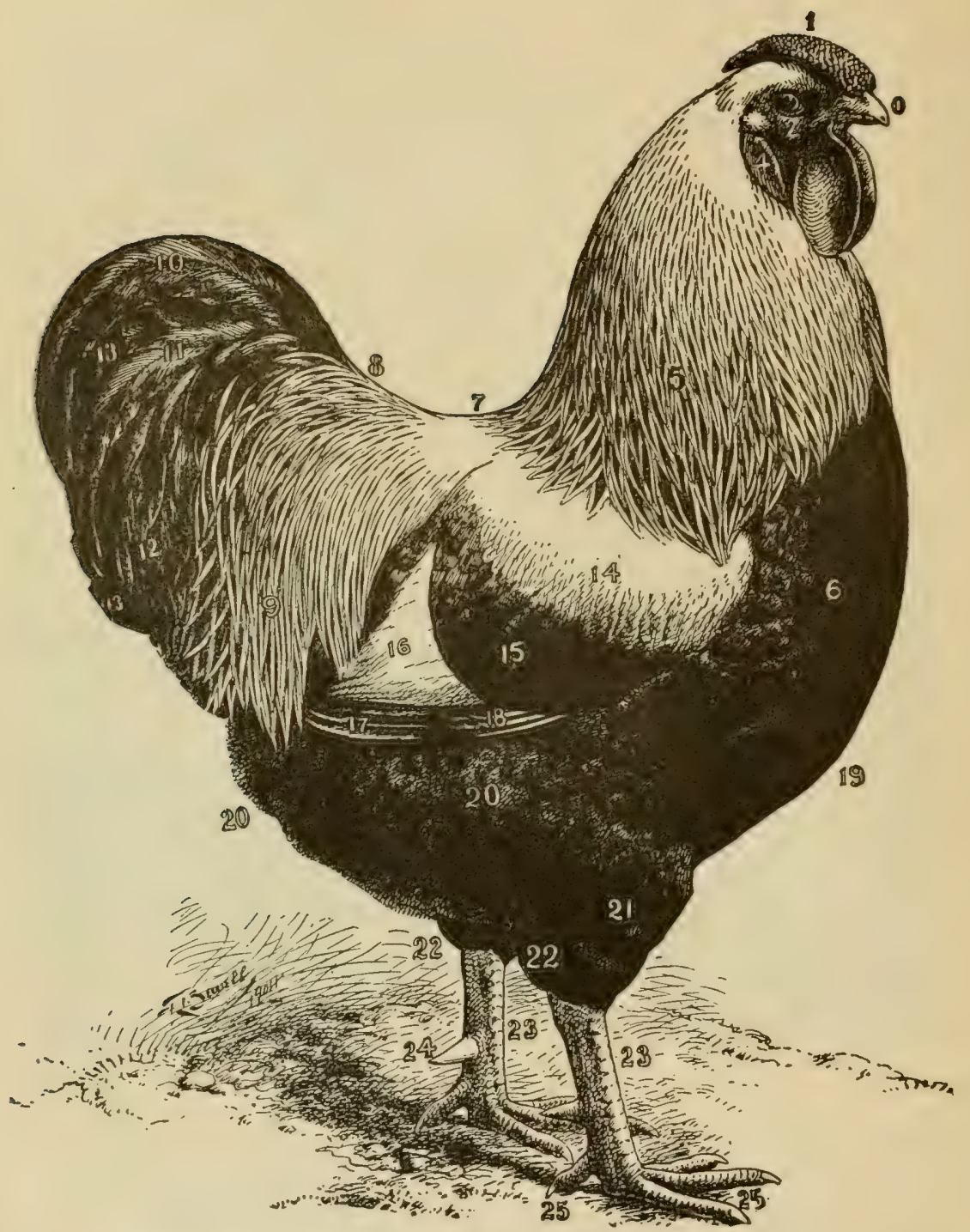

NOMENCLATURE DIAGRAM OF STANDARD FOWL.

0 Beak. 7 Back.

1 Comb. 8 Saddle.

2 Face. 9 Saddle feathers.

3 Wattles. 10 Sickles.

4 Ear-lobe. 11 Lesser Sickles.

5 Hackle. 12 Tail-coverts.

6 Breast. 13 Main tail feathers.

14 Wing-bow.

15 Wing-coverts forming wing bar. 16 Secondaries, wing-bay.
17 Primaries, or flight feathers.

18 Flight coverts.

19 Point of breast bone.

$19,20,20$ Body and fluff.

20, 20 Fluff.

21 Thigh.

22, 22 Knee joints.

23, 23 Shanks.

24 Spur.

25, 25 Toes or claws. 
terested in the goods he offers for sale, and it is in them he will make his announcements.

\section{PREPARING FOR SHOW-ROOM.}

The one feature most required for the proper presentation of the stock in the exhibition hall is to have them hatched from good, healthy stock and properly grown to a complete maturity. No specimen will make an extraordinarily good exhibition fowl that has not been well grown into a strong, vigorous specimen, having fine constitution. This is necessary from the fact that confinement in the show-pen in the exhibition hall is very enervating to all specimens cooped therein.

Presuming that each specimen under consideration has been carefully grown and selected for the purpose, we will attempt to advise our readers of the possibilities of preparing them for the show-room. Always remember, however, that the rules of associations make faking a disqualifiction, and the new rule in the Standard recommends that every specimen shown by the individual exhibiting a fake specimen be discarded from the show-room.

This brings up for consideration the question as to what is "faking." In the language of the new Standard, just issued, faking is described as: Removing or attempting to remove, foreign color in face or ear-lobes when it is a disqualification; removing one or more side sprigs, or trimming a comb in any manner, except the dubbing of Games; artificial coloring of any feather; splicing feathers; injury to plumage of any fowl entered by another exhibitor; plugging up holes on legs of smooth-legged varieties where feathers or stubs disqualify; staining of legs; in fact, any self-evident attempt on the part of an exhibitor to deceive the judge and thus obtain an unfair advantage in competition.

We would refer our readers to page 28 , in the new Standard, as to general disqualifications. This page should be carefully studied by each and every exhibitor.

Every specimen that is sent to the exhibition hall, no matter what color the plumage may be, should be thoroughly tub-washed to gain the best prize. This has been so frequently described that it is scarcely necessary to refer to it again. However, in the washing of fowls, in the preparation for the exhibition hall, there is one feature that demands more than passing attention, and that is the placing in presentable condition the shanks of the smoothlegged fowls. The Standard says: "If the judge thinks that some of the holes or rough places in the shanks are caused by the removal of feathers from the same, the specimen may be disqualified. Often there is a clash between the exhibitor and the judge, the former claiming that rough holes were not caused 
through the removing of stubs or feathers; the judge, thinking otherwise, disqualifies a specimen.

\section{PREPARING THE SHANKS.}

To have a perfectly smooth shank that might otherwise be rough or unattractive, soak the shanks twice a day for several days with kerosene oil. After this has been done, suspend the specimen in a hammock, permitting the shanks to hang down through the holes in the swing. Set this upon a chair or stool; with a bucket of warm water, a woolen rag and a cake of sapolio, polish or wash or rub down to a smooth surface the entire shanks and feet of the specimen with a woolen rag dipped in water and thoroughly covered with sapolio. We have known a patient exhibitor to work five or six hours in this way with a pair of rough shanks until they were as smooth as glass, and as rich in color as it is possible for a shank to be. After these shanks have been thoronghly polished, and placed in the finest condition in this way, wash them absolutely clean with warm water, and anoint them with a mixture of one-half alcohol and one-half sweet oil. Place the specimen in a coop with a perfectly clean floor that may be covered with chaff or cut straw. The following day anoint the shanks again with the mixture of alcohol and sweet oil. Before sending to the show thoroughly tub-wash the specimen, shanks and all, and afterward dry nicely. Never use anything upon the shanks, the face, comb or wattles but pure, cool water. Specimens treated in this way often make a grand appearance in the showroom that might otherwise have been disqualified in bad condition.

The preparation of the specimen for the show summed up in one paragraph is as follows: Perfect health, perfect condition, absolutely free from all possibilities of disqualification of proper weight, shape, formation and color of plumage. If all of these requirements are met to the strongest extent, the specimen is eligible to win. What to feed and how to feed is a question oftentimes propounded. Feed them just as you would to have them in the very best of condition for any other purpose. Never force them into unnatural weight by overfeeding, for an unnaturally fed specimen will lose as much on the cut for condition as he is likely to gain in the weight clause. Try to grow your specimens for exhibition into proper size to meet the requirements of the Standard and make it unnecessary to overfeed them to keep them or have them in proper standard weight.

The time to begin to prepare for the exhibition hall is as soon as the young chicks commence to run about. Carefully look them over and examine them so as to become familiar with their contour and make-up. As the feathers begin to grow, the color and markings will quickly tell those of experience what the prospective quality of those young chicks are, and by giving special care and attention to the best of them you will grow to ma- 
turity a lot of young fowls that will prove to be much better than they would have been if allowed to continue to go on in one flock, all receiving the same attention.

To make a show winner is not the work of a few days or weeks prior to sending them to the exhibition hall. It demands a series of long months of care and attention to bring them to the finest finish, and grow them to a proper size, strength and muscular conformation that is demanded for the best. The present demands of the show-room do not accept medium quality as the best, and it is absolutely necessary to commence early in the game to make next winter's exhibition fowls.

Those who succeed never allow the grass to grow under their feet, as the saying is, but are continually on the watch to have and maintain a flock of exhibition fowls throughout, so that from year to year everything they possess is of that quality which makes the blue-ribbon winners. They never neglect the least or most simple thing they can do that adds to the comfort, growth and handsome plumage of their stock. This is the price that must be paid for quality. The best of quality can not be had in any other way. If you desire to be successful in the show-room, gird on the armor of determination and continued efforts to have them as they should be.

The selection of the best is a problem that confronts every grower of standard-bred poultry. In the first place, the one who selects must be thoroughly conversant with the demands of the exhibition hall and the pranks and self-opinions of the judge, if you will. But above all things remember that you must be almost if not quite as expert at selecting the best before you leave home, as will be the judge who will pass upon them when they reach the show-room.

It is never too soon to begin the proper feeding of the specimens intended for the exhibition hall. These may be frequently chosen when between six or eight weeks old, providing the eye and experience of the selector is equal to the occasion. Such ability only comes after long experience. The reason that the older breeders are the most successful in the exhibition hall is because they have studied the problem, and improve by their experience gained through hard knocks and long-continued care of their poultry at home and in the exhibition hall.

Often we are tempted to select but a few in preparation for the exhibition hall. It is better by far that you should select all the likely specimens at first and colonize them to themselves, and as they improve and grow older, select those of the poorest quality and ship them off for the filling of orders. This will leave in yolir possession the cream of all youl have grown that season, and it is never a difficult problem to sell the best; never be in a hurry to do this, for if you will succeed as an exhibitor and breeder the following season you can not possess too much of the highest 
quality for your matings from which to grow your future stock.

No one feature of an exhibition fowl counts for so much as does the comb and other head points. What might be an otherwise very attractive head is frequently marred by an ill-shaped or crooked comb, or badly formed wattles or ear-lobes. These defects may often be improved. Here is where the experience counts. The working and manipulating of a crooked comb into a straight presentable one, the smoothing out of the wattles, or the flattening down of the ear-lobes becomes as easy to a welltrained manipulator as it does for the surgeon to work into shape any badly deformed portion of the face of a child.

This may be done without being accused of faking. A little glycerin or vasaline taken between the fingers and rubbed against the badly shaped comb may soften and work it into good form. If this is continued for a number of times a defective member may be straightened out and wattles may be improved in the same way. Never be tempted to make use of the knife or scissors to trim or cut in a way that may be apparent to an amateur when he sees it. There are some slight surgical operations that may be performed in this way that are legitimate, but when it comes to cutting for the improvement of formation, it is seldom, if ever, a successful piece of work, for the judge will soon detect it.

Fowls that have feathers on their shanks and feet may be very much improved for the exhibition hall by removing, at least sixty days prior to the exhibition, all the broken, badly-formed or colored feathers, provided they have grown long enough in the shanks and feet to have become hardened, so that the removing of them will not cause a blood flow from the shanks or toes. Old, hard, broken feathers may be removed sixty days prior to the exhibition with almost a certainty that new fresh feathers will have grown in by the time the date of the exhibition is at hand. For the heavy feathering of the large Cochins a little longer time is needed. For others the time mentioned is quite sufficient.

Nothing is better to add luster to the plumage than sunflower seed. We have known Cochins, Brahmas and Langshans to be completely fitted for the exhibition hall with whole corn and sunflower seed. This combination seems to produce long, heavy, fluffy plumage with a fine luster, and plenty of flesh and weight.

TRAINING FOR THE SHOW-ROOM.

It is quite impossible to overestimate the value of good training of fowls intended for the exhibition hall. For the training of these specimens, coops of the proper size, to conform to the size, shape and general make-up of the pens that are to be used in the show-room, should be selected. In this the fowls should be kept, first for a few hours at a time, and then finally confined therein for a day or two in succession until they become perfectly familiar with living within the coop, and lose all timidity from 
being confined therein and handled by those who have them in charge.

These fowls should also be taught not to become frightened at the presence of strangers, women, children, dogs and animals that might possibly visit the exhibition hall where they are to be kept. If you have a lot of exhibition fowls so well trained for the coop that they will scarcely notice the approach of strangers, and will simply look somewhat excited at the presence of a barking dog, you may feel reasonably well assured that the specimens will stand the test of the disorder and surroundings of the exhibition hall.

Considerable trouble comes to those who fail to teach their fowls to stand naturally in the coops and to confront those who approach them rather than to turn from and cower into the corner of the coop. A specimen that will walk boldly up to the judge as he approaches the door of their coop and look at him as much as to say, "Well, here I am for your inspection," wins the admiration and confidence of the judge at once. Those that turn from him and do anything they can to avoid being handled seldom, if ever, gain the much-desired awards.

Teach your specimens that you send them to the exhibition hall to be ever ready and on the alert for some one to open the coop door and handle them. Such a bird is usually designated as a perfectly trained specimen, properly prepared for the examination of the judge and the eyes of the exhibitors. Such a one has many points in its favor, even before a partial examination for quality has been made. The first impression of the specimen in the coop has a lasting influence on the judge so long as he has this specimen under consideration.

For the final finishing touch for the show-room, be absolutely certain that the specimen is thoroughly clean and free from dirt or bad color of any kind. It is always best to thoroughly wash a specimen before it is sent to the show-room. No matter of what breed, variety, or color it may be, it will be greatly improved by a tlıorough washing. Even though this may not be done the comb and head parts should be thoroughly cleaned with luke warm water and a sponge, the shanks and feet thoroughly washed and scrubbed with a good stiff brush, so that when the specimen is shown he will be as clean as it is possible to have it. This is called good condition, and nothing counts for more than this in close competition.

When the specimen is taken to the show-room, always see that it is carefully placed in the exhibition coop several hours before he is to be judged. It is best to coop, water and feed them the night before. Give them plenty to eat and drink at this time. Then, if they are to be judged in the morning, do not feed or water them any more until after they have been judged. 


\section{LINE BREEDING PEDIGREE CHART.}

With many it is a serious question how best to keep a record of their matings, that they may be followed, from year to year, with some degree of accuracy. We believe

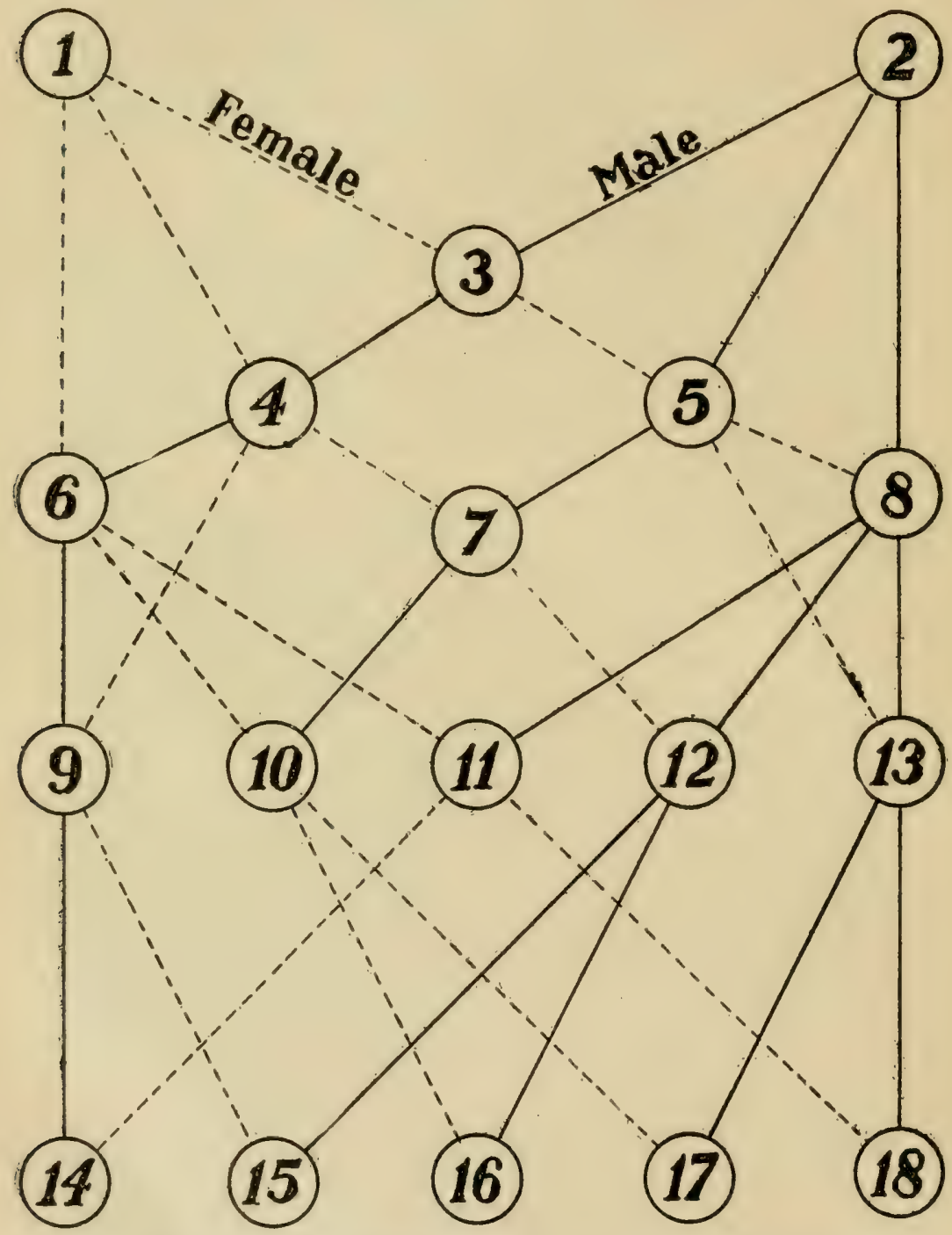

the pedigree chart, shown on this page, fills the bill for this purpose and if one is just starting on a system of line breeding, or in building a strain, it is indispensable. 
"The dotted lines represent the females as having been selected from the upper group, while the solid lines show the male as having been taken from the indicated upper group. Each circle represents the progeny-that is, female No. I, mated to male No. 2, have produced group 3, which is one-half of the blood each of sire and dam. Females from this group mated back to sire (No. 2) produce group 5, which is three-fourths the blood of the sire (No. 2), but only one-fourth the blood of dam (No. I).

"Select a cockerel from group 5 and a pullet from group 4, or vice versa, and the progeny from this group produces group No. 7 , which is mathematically one-lialf the blood of each of the original pair (Nos. I and 2). This is the second step toward producing a new strain. "Females from group 8 mated back to the original sire (No. 2), are seven-eighths of the blood of No. 2. A cockerel from No. 4 mated back to original dam (No. I), produces group 6 , which is seven-eighths the blood of No. I and only one-eighth the blood of the original sire No. 2 .

"A male from No. 6 mated to female from group 4 produces grotp 9, which is thirteen-sixteenths the blood of original dam (No. I), and three-sixteenths of the original sire (No. 2). A male from No. 9 and a female from No. I (the new strain) produced grow I 4 , which is twenty-one thirty-seconds of the blood of the original dam."

If selections are carefully made, choosing individuals of merit in both standard and practical qualities, true to type, the best marked, rost prolific, there is no reason why the percentage of culls will not constantly decrease and the beauty and productiveness constantly increase, where environment, care and feeding are carried forward with the same intelligent forethought that characterizes such a selection and mating of standard-bred fowls. 


\section{Crate Fattening of Chickens.}

We have many inquiries each day for our method of crate fattening and our formulas, and we can assure our readers that the trouble and expense is small compared with the increase in flesh and the price obtained for these birds. The primary object in fattening chickens is not to produce excessive fat, as might be inferred from the term "fattening," but to cover the chickens with finely flavored, edible meat. Many of the experiment stations, as well as our personal experience, determine the age to most profitably fatten to be not less than three months, and in all

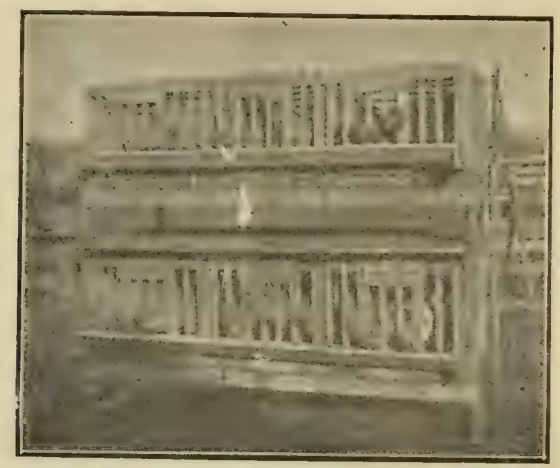

Crates for Fattening Birds.

but the Mediterranean classes they can be profitably fattened as old as eight months. We refer to cockerels in placing this age limit, as pullets and hens can be used much older to advantage.

The regulation crates hold twelve chickens each. The frame of the crate is six feet long, sixteen inches wide and twenty inches high. It is built from $7-8$ by 2 inch dressed lumber, and is divided by two tight wooden partitions into three compartments; each compartment holds four chickens. The frame is covered with slats; the slats are placed lengthwise on the bottom, back and top of the frame, and up and down in front. The slats can be ordinary four feet laths or one-half inch strips sawed from a board $7-8$ inch thick. The bottom, back and top slats are put on one and one-half inches apart. The spaces between the slats in front are two inches wide, to enable the chickens to feed from the trough. The top slats are cut above each partition and six strips two inches wide are nailed under them. The three doors so formed are hinged to the rear corner piece of the frame. The crates are 
placed on stands, fifteen inches from the ground. The droppings from the chickens are received on sand or other absorbent material. A light " $V$ " trough, two and one-half inches inside, is placed in front of each crate, and is carried on two brackets nailed to the end of the crate. The bottom of the trough is four inches above the floor, and the upper inside edge is two inches from the

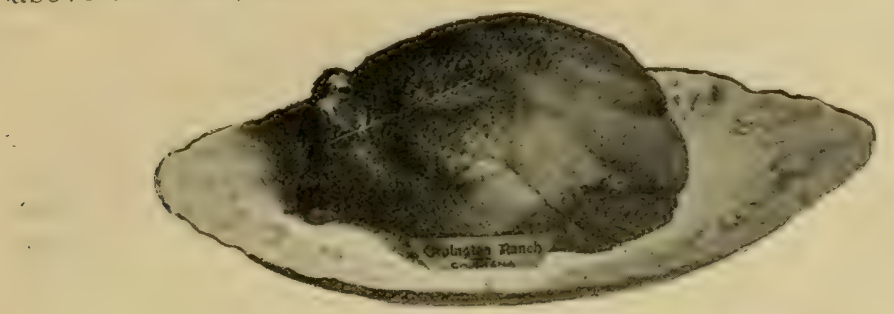

Ready for the Oven, Crate Fattened Orpington.

crate. The crates should be so situated that the chickens will receive abundant ventilation at all times. Dorking, Orpington, Inclian Games, the Rocks, II yandottes, in fact, almost any of the heavier breeds, fatten more profitably than the Mediterranean classes. Suitable chickens for fattening are those of medium size, three to four and a half pounds each, of a broad, square shape, with short, straight legs, set well apart. Large framed chicliens,

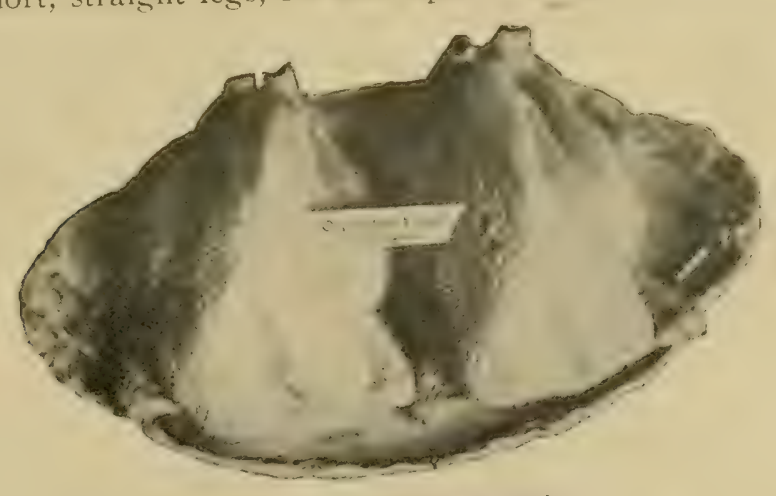

Crate Fattened Fowls.

with prominent breast bones, cannot be so satisfactorily fattened. Ground oats, finely ground, or with the coarser hulls sifted out, should form the basis of all the grain mixtures. Ground corn can be fed in a limited quantity - not more than one-fifth of the grain by weight. Ground corn fed in excess will result in a yellow flesh.

\section{SATISFACTORY MEAL MIXTURES.}

I. Ground oats (coarser hulls removed). included). 
3. Equal parts ground oats, ground barley and ground buckwheat.

4. Two pounds ground oats, two pounds ground buckwheat, one pound ground corn.

The ground meal should be mixed to a thin porridge with thick sour skim-milk, or buttermilk. On the average, Io pounds of meal require from fifteen to seventeen pounds of sour skim-milk. A small quantity of salt should be added to the mash. When st:fficient skim-milk or buttermilk cannot be obtained for mixing the mashes, a quantity of animal and raw vegetable food should be added to the fattening ration.

The most profitble period for fattening is about twentyfour days.

It is well to divide this period into the first and second weeks, during which time the chickens are fed the ground meal and skim-milk mashes; and the remaining ten days when tallow should be added to the mashes. in crates.

The chickens should fast for one day after being placed

The chickens should be free from lice.

Before the chickens are placed in the crates, they require a thorough dusting with insect powder or sulphur. They should be again dusted three days before they are killed.

The chickens should be fed sparingly the first week.

A small quantity of the fattening food should be spread along the troughs, and as this is eaten, more food is added, but not as much as the chickens would consume. The food should be given three times a day, and after feeding the troughs should be cleaned and turned over. The chickens should receive in the trough fresh water twice a day, and grit two or three times during the week.

The second week the chickens should be given as much food as they will eat.

They should be fed twice a day. Half an hour after feeding, the feed troughs should be cleansed and turned over. Water and grit should also be supplied as in the first week.

The last ten days the chickens should be fed as in the second week. Tallow should be added to their ration.

At the commencement of this period, one pound of tallow a day should be added to the mashes for every seventy chickens. The quantity of tallow is gradually increased, so that at the latter part of the period one pound of tallow is fed to fifty chickens. This paste can be mixed with the mashes. The chickens should receive the fattening food twice a day, and also water and grit.

The chickens should be starved 36 hours before killing.

This will prevent food remaining in the crop and in- 
testines, which would decompose and spoil the flavor of the birds. Several hours after the last feed allow the chickens what water they wish to drink. They should then have a complete fast until thev are killed.

The chickens should be killed by dislocating the neck.

\section{DIRECTIONS FOR DISLOCATING THE NECK.}

With the left hand hold the chicken's legs and wings in one firm grasp. Place the first finger of the right hand on the right side of the neck and the remaining fingers on the left side. Grasp the head in the hollow of the hand, with the fork of the fingers behind the head where it joins the neck. The back of the chicken being upwards, hold the legs against the left hip and the head near the right thigh or knee. Bend the head backwards as far as possible, and at the same time stretch the neck, when it is dislocted immediately; pull the head about one and one-half inches from the neck. Hold the wings firmly after killing, and allow the chicken's head to hang down, so that the blood can collect in the neck; the head is attached to the body simply by the skin of the neck.

ADVANTAGES OF THIS MODE OF KILLING.

The body of the chicken is as free from blood as when any other method of killing is employed. The blood of the chicken remains in the one and one-half inch space between the head and the neck; the weight of blood will pay for the plucking. Plucking is not an unpleasant operation. Air is not permitted to enter and dry out the chicken.

The chicken should now be dry plucked. Plucking should be commenced as soon as the chicken's neck is dislocated. The chicken is not drawn.

\section{DIRECTIONS FOR PLUCKING.}

While still holding the chicken in the left hand, extract the tail feathers and the quill feathers of the wing. Allow the chicken's head to hang down, and commence plucking the feathers on the back and wings; then pluck the breast and lower part of the neck, and work back on the body to the tail and turning the birl over again, finish the back and wings.

Remove all pin feathers and make the chicken as attractive as possible. Use care in plucking so as not to tear the skin. If a tear is made, have the flesh brought together with white thread.

If the chickens are to be shipped to market, they must be thoroughly cooled and dry on the skin before they are placed in the shipping cases. Unless the chickens are arti- 
ficially cooled, they should not be packed into the cases until twenty-four hours after killing.

\section{CROSSES FOR MARKET STOCK.}

In the chapter on "Breeds Best Adapted to California" we have treated at some length on the standard varieties best adapted for meat purposes. While this list covers a!most every requirement of the market poultryman, it is often found profitable to make what is known as "one-year crosses," that is, cross two standard varieties and use their offspring for market purposes only and not allow the crossing to go further. As a rule a cross that is properly balanced will be a quick grower and make an excellent bird to put on flesh in the fattening pen. After considerable experimenting we have found that any of the English or American varieties crossed on the Asiatic are much larger than the straight-bred English or American bird. In this cross it is best to use the male of the smaller breed on hens of the larger variety. For example, take half a dozen Light Brahma females of good: size and mate them to either a Silver Grey Dorking, White Orpington or White Rock male and the result wil! be a fine lot of birds larger than the male but having a much plumper and more easily fattened carcass than the Brahma female. This cross is intended to produce soft roasters, as birds from such a mating will not give the quick feathering desired for broilers or fryers.

Another good cross is the Indian Game male crossed on Asiatic females for soft roasters, or Cornish Indian Game females mated to a Brown Leghorn for broilers or fryers. A male of any of the Mediterranean varieties crossed on any of the American or English classes produces an excellent broiler or fryer. We have found a cross of a White Leghorn male crossed on White or Barred Rocks, White or Buff Orpingtons or White Wyandottes produced the best of fastgrowing and quick feathering broilers and fryers. Of course all these birds can also be carried to the soft roaster age and make fine birds for the smaller soft roaster demand. While we believe that the standard varieties cover the demand for the dressed or live poultry market we also know that proper crossing often pays the breeder. One mistake that many make is in breeding back to these crosses the second season. It is impossible to carry on breeding operations with the cross-bred birds after the first season without loss to the breeder. 


\section{Caponizing. What Are Capons?}

Capons are aptly termed the "finest chicken meat in the world," for there is nothing growing feathers their equal or superior. A capon is neither a rooster nor a hen-it is nothing else than a capon. After removing the testicles from the cockerel, its nature becomes entirely changed. They take on a more rapid growth, are more tame, awkward in carriage and always exceedingly lazy, take on' a very heavy and beatififul plumage, the comb and wattles cease to grow, the spurs do not develop as in the cockerel, and being cast off by both rooster and hen, he soon shows a fondness for the society of little chicks.

\section{BEST TIME, TO CAPONIZE.}

Fowls hatched any time of the year make fine capons; no ill results follow the operation at any time of the year. The bird should be from two to three months old (not over six months), and weigh not less than a pound to a pound and a half. The size is equally as important as the age. April, May, June, July, August, September and October are the months generally taken for caponizing, for the reason that spring chickens arrive at proper age and weight, during these months; also because cockerels caponized then arrive at the proper age and weight during the months of November, December, January, February, March, April and May, at which time there is the greatest demand for them in the cities and highest prices secured.

\section{PROFIT IN CAPONS.}

Caponize the chicks and you have at once laid the foundation for a handsome profit for some time to come. Outside of the cardinal points of profit, the simplicity of the operation (when proper instruments are used) recommends itself to everyone. A boy ten years old can readily perform the operation, and anyone can soon become an expert.

To the poultry raiser we would say we know of no source of profit bringing larger returns for the outlay than raising capons, the profit in a great majority of cases being over 100 per cent. The question of assured profit is an all-convincing argument in any line, and pre-eminently so to the poultrymen, whose losses are added to from various unlooked-for sources. 


\section{DIRECTIONS FOR CAPONIZING.}

From twenty-four to thirty hours before performing the operation, select such cockerels as you intend to caponize, confining them in a clean, airy coop or room without either food or water. The best time to confine them is at early morning, as their long fast will then end about noon of the following day, at which time the operation is performed. Should the day be cloudy or wet, do not caponize them, but let the operation go until you have a bright and fair day. It is necessary that you have all the light possible in the matter. Now, after slightly wetting the spot, proceed to turn down the feathers from the upper part of the last two ribs and just in front of the thigh joint. Pull the flesh on the side down toward the hip, and when the operation is finished the cut between the ribs will be entirely closed by the skin going back to its place.

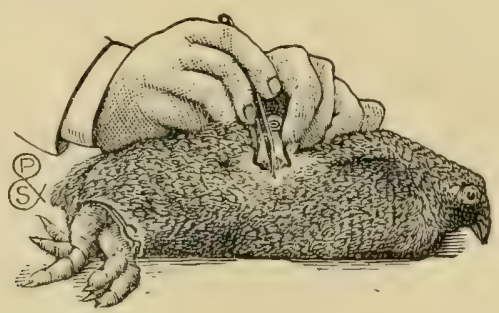

MAKING INCISION. PROPER INSTRUMENTS.

We have laid considerable stress upon having proper instruments in caponizing, and the more we read of the literary effusions appearing in the numerous papers today touching caponizing instruments the more need we feel there is to caution the inexperienced ones. While it is not cruel to caponize, it is inhuman to butcher or to cause unnecessary pain.

\section{CAPONIZING IS NOT CRUEL.}

A large number of persons hesitate in caponizing, feeling it to be cruel to the bird. To these we wish to bring our experiences in this matter proving to the contrary. This is a greatly mistaken notion, and the operation bestows an unlimited amount of kindness on the bird, even if there were no other considerations or return. The writer has seen cockerels fly at one another time and again, tearing flesh and feathers with beak and cutting with spurs. Before the combatants could be separated there has been: a disfigured comb, probably a blinded eye and a generally cut-up bird. This is the essence of cruelty. 
INSERTING SPREADER.

After caponizing, the habits of the bird are entirely changed. Their disposition is quiet and peaceable, habits mild and tending to a solitary life and perfectly contented wherever situated. They no longer chase about the farm spoiling for a fight and running off flesh as fast as put on. They no longer arouse the whole neighborhood from morning until night with their incessant crowing, but, on the conr

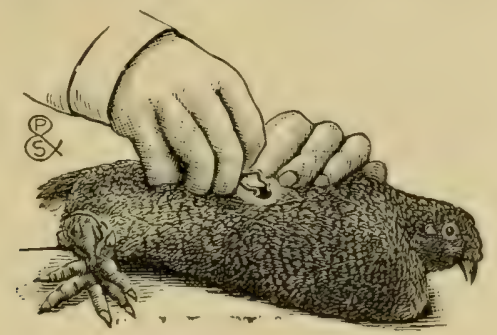

trary, become models of good dispositions, leading a quiet life that will surely bring large returns to the raiser. An operation that does away with so much inborn evil cannot be considered cruel.

REMOVING TESTICLES. TREATMENT AFTER OPERATION.

Beginners have an idea that the caponizing operation is a very severe shock to the bird and a great drain on its vital organ. This is a mistake. The testicles are not a vital part. Their removal is of little consequence so far as the bird's health and vitality are concerned, the only difference it makes is in regard to the sexual development of the bird. The thin

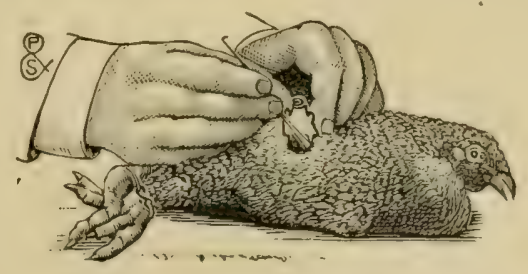

membrane which envelops the intestines is not a vital part. The holes which we have torn in it are an injury which amounts to almost nothing. The only real injuries inflicted, therefore, are the two incisions, and these are merely flesh wounds of the simplest character, and by no means severe. The skin, having slipped back in its natural position, covers the flesh wound between the ribs; the cut in the skin, and that in the flesh, on each side, cannot well be in a more favorable 
shape for rapid healing. There is no need of sewing the edges of the wound together, or using any kind of salve, or plaster, or wash. Just trust in Nature, the best physician, and you will not be disappointed.

At the beginning of the caponizing season, a "capon hospital" might be put up. This is simply a space containing, say, two or three square feet of floor surface to each capon, and covered with a low roof as a protection from rain and sun, and tightly enclosed with wire netting. Inside is a coop in which the convalescent fowls spend the nights. A box in one corner is kept well supplied with soft food (bran and meal moisted with skim-milk), and a dish in the other corner contains the water which should be frequently renewed. Some of our instructors tell us to feed lightly as first; others advise giving all the food that the birds will eat. Just as soon as a capon is put into the "hospital," and once gets sight of the feed-box, he will forget all the trials which he has just experienced, and at once proceed to fill his crop. The birds may be confined in close quarters for at least eight days after the operation. Many of them "wind-puff" badly, a lot of air gathering under the outside skin and giving the capon a puffed-up appearance and probably causing much inconvenience. Look the confined birds over once a day, and give speedy relief where needed by pricking the puffed-up skin with the point of a keen penknife. Part of the birds do not need this attention; others wind-puff right along for a week or so, and need frequent touches with the penknife. Usually one can tell by the appearance, and always by the feeling, whether there is wind-puff and cause for treatment. In consequence of this confinement, of the treatment and of their voracious appetite, the capons become exceedingly tame and tractable.

The straw, leaves or soft earth on the floor of the "hospital" should, for the sake of cleanliness, be often renewed.

When the period of convalesence (eight or ten days) is past, the capons should have their liberty. They will not wander off very far, but stay most of the time near where they are accustomed to get their regular rations. At night drive them into the "capon house," a warm stall with low roosts, regularly cleaned and disinfected. Capons do not seem to be particular about their roosting place.

How many capons may be crowded together in one building seems to be a disputed point. Some experts claim that 40 to 50 is the limit of safety. We have had 80 to IoO roosting together in a rather small house, the capons having free range in day time, with satisfactory results. 


\section{THE BEST BREEDS FOR CAPONIZING.}

The selecting of the proper birds for caponizing is an important matter. Since one of the main benefits, derived from caponizing is the increased size of the bird, it is necessary that we select birds that are naturally of a large bone and frame. This cuts all of the Mediterranean varieties out of the class suitable for capons and leaves the American, English, Asiatic, French and Game classes as the best breeds to select from.

From an experience with all of the latter classes, extending over several seasons as regards their value as mature capons, we have found that the Orpington, Rock, Wyandotte or Indian Game cockerels make by far the largest and best capons of the standard varieties. In the chapter on "crosses" we have described a number of mixtures that make extra quality capons. In fact all of those given as "roaster" crosses will produce cockerels that if caponized will make the highest quality in the capon market.

\section{CALIFORNIA AS A CAPON MARKET.}

A few years ago capons were unknown in the California markets, but thanks to a few enterprising poultry raisers and a demand for the best in poultry meat by the tourists from eastern cities, they are now in great demand and the supply is considerably below the demand. The many large tourist hotels scattered over Southern California will consume all the capons turned out for some years to come. We know of one of these hotels that offered to contract with a breeder in Los Angeles for 2000 capons to be delivered during the coming winter. This was only one of many that ronld gladly use capons if they could secure them. Besides these are the hundreds of private families with large means that want the best in everything and would gladly use capons instead of the tough roasters they are compelled to use at present. Prices ranging from $35 \mathrm{c}$ to $60 \mathrm{c}$ per $\mathrm{lb}$. are paid for first-class dressed capons, and when we con. sider the fact that they are the cheapest of all fowls to raise and fatten, and that the operation increases the natural weight of the birds from 5 to $8 \mathrm{lbs}$., it will be seen that there is a handsome profit in producing capons for the California markets.
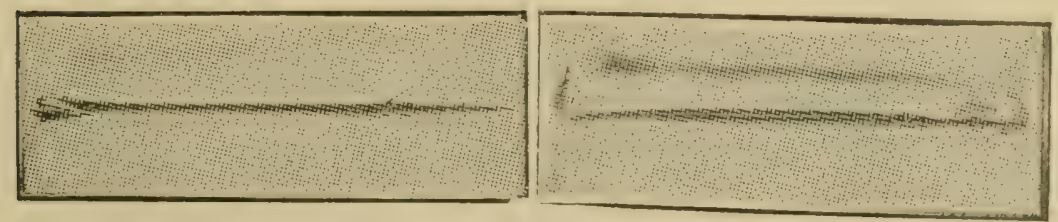

The Selfridge Caponizing Spoon. 


\section{Lice and Mites.}

In our mild climate lice and mites thrive the year around and the successful poultryman must keep everlastingly after them to keep his birds in the best condition. These pests are no harder to keep in check here than they are elsewhere, but with our mild winters it is necessary to keep up the war of extermination every month in the year. If one keeps the vermin matter well in hand there is very little work attached to it, but once let a poultry plant get infested and it takes a great deal of hard work to exterminate the pests.

The louse plague is the result of contagion. The parasites are introduced upon birds, and they multiply and increase to a remarkable extent when the conditions are favorable. It has been estimated that the second generation from a single louse may number twenty-five hundred individuals, and the third generation may reach the enormous number of one hundred and twenty-five thousand; and all of these may be produced in the course of eight weeks. It is not surprising, therefore, that, although birds have so few parasites upon them when well cared for that these insests can not be discovered, a few weeks of neglect and unfavorable surroundings may bring an entire change and reveal them covered with vermin, exhausted and emaciated.

The different species of lice and mites are as easily distinguished from each other by experts as are the different species of birds. Their peculiarities are inherited from generation to generation, and they continue to live upon the same species of birds. The hens, the turkeys, the pigeons, the ducks, the geese, and the Guinea fowls each have their peculiar species of lice which live upon them alone, and there are but comparatively few kinds of lice which live upon two or more species of birds. We may, consequently, favor the multiplication of lice by making the conditions favorable for them, but we can not produce lice where none exist.

The conditions which favor the multiplication of lice and other external parasites are found partly in the birds and partly in the surroundings. Unhealthy or unthrifty birds, or those which from any cause lack strength and vigor, are most subject to the attacks of such parasites, and may be found literally covered with them when other members of the flock are comparatively free from their attacks. There are birds so strong, healthy and vigorous that lice can not thrive upon them. Exercise, proper feeding, pure air, all have a tendency to keep the 
birds in a condition unfavorable to the existence of the parasites. On the other hand accumulations of manure and filth, close confinement of the birds, lack of dusting places, buildings with crevices in which the insects may hide, and damp, dark and badly-ventilated houses are conditions which favor the parasites and lead to their rapid development.

The poultry yard may be kept practically free from these insect pests, but there is always a liability of their introduction with purchased fowls or with birds that have become infested at shows and other places. Contagion must, therefore, be accepted as the cause of this plague, and it should be guarded against on the same principles as are the infective elements of other communicable diseases.

Young chickens hatched under hens almost invariably have lice upon them and should be treated as soon as removed from the nests. An efficacious and safe remedy in this case is pure lard. It should be carefully rubbed into the feathers upon the top of the head and under the throat. Lard obstructs the breathing pores of the lice and soon kills them. Nothing should be mixed with the lard to increase its activity as such young birds are very sensitive to the action of irritants and are seriously injured by them. Some recommend mixing powdered sulphur with the lard for this purpose; but it should on no account be used on small chickens as it causes inflammation of the eyes and arrests the growth. Pure lard is harmless and will kill the lice, consequently it can be used with full confidence. A small quantity may also be rubbed under the wings, but this is not usually considered necessary until the chicks are a week or two old.

Chickens hatched in the incubator should be free from lice, and will be unless the incubator has in some way become infested. It is well to examine even incubator chicks occasionally for lice as the incubator or brooder may have these insects introduced into them in various ways and then become favorable places for the growth of the parasites as well as for the development of the birds. The older birds, but not the hens with young chickens, may be anointed about the heads, under the wings, and around the vent with an ointment made by thoroughly mixing a teaspoonful of flowers of sulphur with an ounce of lard. Some use instead of this ointment or in combination with it insecticide powder, which is blown or dusted into the feathers. This operation is best performed by holding the bird by the legs, head downwards, so that the tendency of the feathers will be to fall away from the body. Then apply the powder thoroughly with a dredging box or a powder bellows. If the bird is held over a large piece of paper the powder which fails to adhere to the feathers and skin can be saved and used a second time. It is 
considered a good plan to moisten the roots of the feathers with soapy water in order to make powders adhere. Some poultrymen dip their fowls in a solution containing one per cent. carbolic acid. This solution is made by mixing I I-4 ounces of pure carbolic acid with I gallon of hot water. Larger quantities may be made in the same proportion. The solution is allowed to cool and is then put in a vessel suitable for immersing the bird to be treated. The fowls should be held in this liquid for about a minute and care should be taken to wet every portion of the body and head. Creolin will probably prove more satisfactory for this treatment than carbolic acid, as it is equally efficacious in killing insects, but is less poisonous to birds and the odor is less objectionable to the operator. It is used in the strength of $2 \mathrm{I} / 2$ ounces mixed with a gallon of water.

The treatment of the birds with some of the substances mentioned, although it is indispensable for the control of the parasites, is not alone sufficient. The buildings must receive prompt attention and thorough treatment. The droppings should be carefully removed and mixed with fine, dry road dust, ashes or lime. Fine dust is destructive to the lice and hence should be plentifully used about the buildings, and given to the fowls in boxes so large that they can thoroughly dust themselves in it. Pyrethrum and sulphur may be mixed with the dust in such boxes and render it more deadly to the insects.

The floor of the poultry house or pigeon cote should be thoroughly scraped, and covered with fresh sand or road dust. The roosts and all loose pieces of wood should be removed and then the whole inside and also the parts which have been removed should be well wetted by spraying with a solution made by dissolving 6 ounces of crude carbolic acid to the gallon of water. This should be mixed with the water at nearly the boiling point as carbolic acid does not readily mix with cold water. At least once a year the inside woodwork and walls should be given a good coat of lime wash to which 4 ounces of crude carbolic acid for each gallon has been added. The nests should also be cleaned out, drenched with boiling water or carbolic solution, and when dry sprinkled with insecticide powder and bedded with fresh straw. The carbolic preparations should always be used in the morning and the buildings thoroughly ventilated during the day so that the odor will not be so strong as to be injurious to the birds at night.

The treatment recommended above, if thoroughly carried out, is sufficient to keep the troublesome external parasites either eradicated or under such control that they will not prove injurious. For convenience various other methods have been devised which are more or less effectual. Some throw dry lime dust against the roof and walls of the buildings; others fumigate 
by burning sulphur and leaving the doors and windows closed for a few hours, there being, of course, no birds left inside at the time; still others fumigate by placing open bottles of bisulphide of carbon about the poultry house. Aniseed is distasteful to most of the vermin of birds, and is an excellent remedy when powdered and dusted into the feathers, or when strewn into the nest boxes. A small quantity of the essential oil mixed with hot water renders this more effectual in destroying the mites about the roosts, nests and other woodwork.

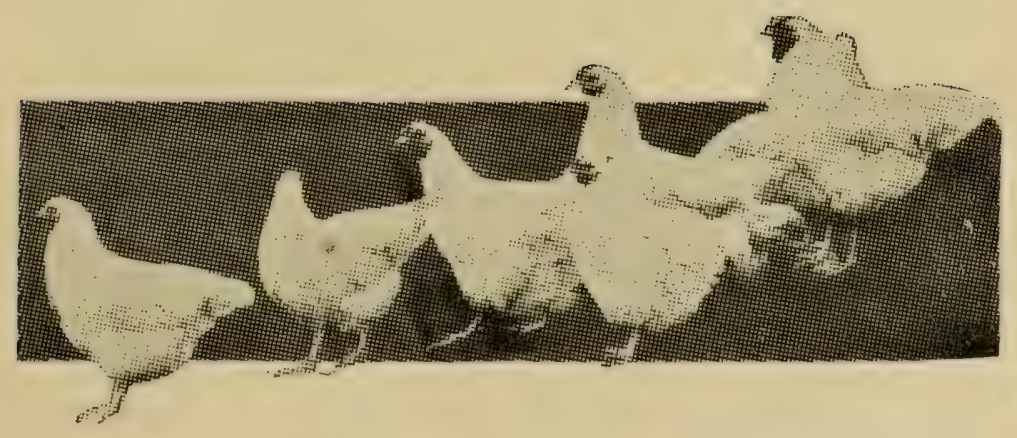




\section{Diseases and Cures.}

\section{COMMON CAUSES OF DISEASES.}

In order to act intelligently for the prevention and cure of a disease, we must know something of its cause; and in order to determire the cause, we must know the agencies which are liable to injuriously affect the diseased organ. If it is found that the digestive organs are affected, we are naturally led to inquire into the character and quantity of food that has been taken; the amount of exercise which the birds have had; whether they have had access to gravel or grit; whether the alimentary canal is obstructed at any point; whether the affected birds have been unduly exposed to cold or to draughts of air; and, finally, whether they have been attacked by any of the parasites, vegetable or animal, which are known to cause disturbance of the digestive functions. If the respiratory organs are diseased, it is proper to inquire if the birds have been chilled or exposed to draughts of air, or to a damp atmosphere; or whether they are the prey of parasitic organisms such as multiply in this portion of the bird's body. If the skin, comb, wattles, or feet are affected, the most likely causes are mechanical injuries and parasites. If lameness is exhibited it is probably due to injuries or rheumatism. If the general health is affected as shown by mal-nutrition, paleness, and loss of weight, the most common causes are indigestion from improper food, and the attacks of internal or external parasites. Diseases of the brain result from exposure to too great heat, and sunstroke, from over exertion, and from too high feeding with insufficient exercise. Diseases of the ovaries and oviducts result from the bird being too fat; from these organs being over stimulated, or from the ration being improperly adjusted to the needs of the body.

\section{OBJECTS OF MEDICAL TREATMENT.}

In the treatment of sick birds, medicines should not be administered blindly, but on the contrary there should be a clear idea, before the remedy is selected, of what is to be accomplished. Othervise, it is probable that more harm than good will result. In giving medicines intelligently there are three distinct objects which one may attempt to accomplish:

I. To neutralize, remove or destroy the cause of the disease.

2. To cause the repair of the affected tissues.

3. To counteract or remove the symptoms. 


\section{SIMPLE CATARRH.}

One of the most common diseases of birds is catarrh. It is sometimes mild, attacks but a few birds in a flock and is easily overcome. At other times it is more severe, affects all or nearly all the birds in a flock and only yields to energetic and prolonged treatment. In case of long-continued cold and damp weather, birds in different flocks may be affected at the same time, leading to the suspicion of an infectious disease, although it is really due to climatic conditions acting over a large territory at the same time. It is important to make a careful examination as soon as the disease appears and to determine whether it is simple catarrh or infectious catarrh, as the treatment to be adopted in the two cases differs materially.

In simple, non-contagious catarrh, the affected birds are more or less dull, they are disinclined to move, their appetites are diminished, they sneeze and the mucous membrane is thickened, causing some obstruction to breathing through the nostrils. There soon appears a thin, watery discharge which later becomes thicker and glutinous, the eyes are often watery, the eyelids swollen and sometimes held together by a thick, viscid secretion. In yery severe cases, the birds are somnolent, the plumage is erect and roughened, the nostrils are completely obstructed by the thick secretion, the breathing is entirely through the mouth and is accompanied by a wheezing or snoring sound, the appetite is entirely lost, a thin liquid escapes from the mouth and the bird soon becomes exhausted and dies. In the milder cases simple catarrh may disappear without treatment, in the course of two or three days; or, in case the cause is allowed to persist, the birds may become worse and die or the disease may become chronic and continue its effects for a long time.

TREATMENT.-Catarrh is best prevented by keeping the birds in a strong, vigorous condition through proper exercise, good food, clean houses and ventilation without draughts. In case symptoms of this disease are observed, search out and remove the cause, and give the birds stimulating food with some cayenne pepper mixed into it.

If the disease appears serious, wash out the mouth and nostrils twice a day with horic acid solution of the strength of fifteen grains to the ounce of water, or creoline solution (one per cent). The following remedy is highly recommended by Megnin:

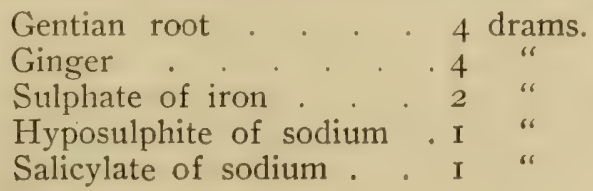


These substances are to be pulverized and then thoroughly mixed. The dose is three or four grains a day for a mediumsized fowl. Its effects are stimulating and tonic.

\section{ROUP, CONTAGIOUS CATARRH.}

The early symptoms of this disease can not be clearly distinguished from those of simple catarrh, though the dulness and prostration are usually greater and there is often a peculiar offensive odor, which are valuable points to aid in reaching a decision. There is at first a thin, watery discharge from the nostrils with some obstruction to breathing from swelling of the mucous membrane. The discharge soon becomes thicker and more sticky or glutinous, causing the affected birds to sneeze and shake their heads in an effort to free the air passages. The subjects are dull, quiet, without much appetite and more or less feverish. The plumage is rough, the wings pendant, the comb and wattles dark red.

As the disease advances, the inflammation extends from the mucous membrane of the nostrils along the internal surface of the passage connecting the nostrils with the orbital space or cavity surrounding the eyes. The orbital space in birds is relatively very large and has an angular projection towards the point of the beak. There is, consequently, considerable space between the eye-ball and the bony walls surrounding it, which is known as the orbital space or orbital sinus. This space is lined with a mucous membrane which is continuous with that of the nostrils and in such close relations with it that an inflammatory process readily extends from one to the other. Very often in roup, this membrane is inflamed throughont these passages, and the process is so acute that the surface becomes eroded and ulcerated. The secretion, instead of remaining fluid as in simple catarrh, changes at this period, is abundant, thick, yellowish, purulent, and cheesy. This secretion accumulates and obstructs the passages. It forms around the eye and forces the eye-ball out of the socket and at the same time collects under the eyelids or on the corner, sometimes simulating a false membrane. It, also, plugs the nasal passages, presses down the palate, obstructs the breathing and may prevent swallowing.

The affected birds in the advanced stages of the disease present a most miserable appearance. The beak is kept open in order to breathe, the sight is destroyed by inflammatory products, the eyes are often closed and bulging from the head, the breathing is very difficult, a thick, sticky discharge escapes from the nostrils, the subjects are emaciated, prostrated, sleepy and perhaps unconscious. When these severe symptoms appear, the strength is soon exhausted and death follows.

Contagious catarrh spreads rapidly through a flock, affecting the greater part of the birds and causing the death of a variable 
proportion according to the virulence of the contagion. It is always a very serious disease and is difficult to eradicate.

That roup is a contagious disease admits of no question:Its origin in a flock can frequently be traced to the introduction of a bird from some other premises where the disease exists. Often, it is contracted by exposure to affected birds at shows, or to the contagion clinging to coops, houses or in: fected runs. Many writers teach that roup may originate from exposure to cold or draughts of air. This prevalent opinion is probably accounted for by the fact that the first stages of roup are almost indentical in their symptoms with simple catarrh or a common cold. It is not likely, however, that such exposure is sufficient to produce roup. Contagious diseases are caused by parasites, each specific disease having its particular species of animal or vegetable life to call it into existence, and without its particular parasite the disease cannot be developed. It is, therefore, logical to conclude that if the germ or parasite of roup is not already infecting a given poultry yard, the disease will not appear until this parasite is introduced. Colds and catarrh may become aggravated and even fatal by neglect and continued exposure to the existing causes, but they will not develop into roup unless the contagion of the last-ramed disease is present.

The measures to be adopted for the prevention of roup are the same as for other contagious diseases. Exclude the contagion by every means that can be devised. Do not allow poultry, animals or men! to come directly from an infected premises to a healthy flock. Be careful in buying new birds, and these as well as all birds that have been to shows should be isolated for two or three weeks until their healthfulness is demonstrated. If the disease once appears in the flock use disinfectants freely and isolate diseased individuals. If the birds are not very valuable it is sometimes better to dispose of every one, and after disinfection begin with new blood. The contagion is persistent and can only be eradicated by the most thorough measures.

The medical treatment may be undertaken with good chances of success, but requires time and constant attention. Whether it will pay, depends upon the value of the birds and of the services-of the party who cares for them. Antiseptic and healing applications are indicated to the interior of the nostrils, the cleft of the palate, the eye, and other affected parts. If swellings containing thick pus or cheesy matter form upon the head, they should be opened with a sharp instrument, the contents removed and the wound treated with antiseptics. Antiseptic powders are convenient for treating the wounds caused in such operations. Among these iodo- 
form and aristol are the best. The wound may be kept filled with either of these powders until it is healed. The antiseptic solutions may be injected into the nostrils or into the cleft in the roof of the mouth which communicates with the nostrils, by using a small syringe, a medicine dropper, or even an oil can, such as is used for oiling machinery. A syringe is, of course, the best. The solutions most suited are boric acid fifteen grains to the ounce of water; carbolic acid or creolin in two per cent. solution; peroxide of hydrogen diluted with an equal quantity of water; permanganate of potash one grain to the ounce; or sulphate of copper five grains to the ounce. Some persons have reported great success from the use of coal oil (kerosene) either pure or diluted with equal parts of olive oil. Megnin has had excellent results with a five per cent. solution of sulphate of copper injected into the nostrils, the cleft of the palate, the affected eyes and the wounds made in removing purulent collections from the sinus in front of the eyes. This solution appears rather strong and should be used at first with care and in: an experimental way until its effects are seen. When the head shows signs of considerable inflammation it may be anointed with sweet (olive) oil, vaseline, or equal parts of sweet oil and coal oil.

In selecting from the remedies mentioned, it is well to begin with the milder ones, such as boric acid, peroxide of hydrogen or carbolic acid. Keep the birds comfortable and free from draughts of air. Give stimulating and easily digested food containing a small proportion of meat scrap. It is important to dispose of the carcasses of dead birds promptly by burning or deep burial.

\section{BRONCHITIS.}

Inflammation of the mucous membrane of the trachea and bronichial tubes, or bronchitis, is sometimes due to the extension of the catarrhal process from the head, or it may be induced by the action of the causes which produce catarrh. The birds are somewhat dull, with loss of appetite, accelerated breathing and cough. On listening to the respiration a blowing or whistling sound may be heard in the air tubes, in the early stages of the disease, caused by the air being drawn over the dry and thickened membrane; later, a rattling or bubbling sound is caused by air passing through accumulations of mucous. In the majority of cases the symptoms are not serious, and, under favorable conditions, they soon disappear. Occasionally, the malady increases in intensity, the plumage becomes rough, the wings droop, the breathing is labored and difficult, the bird frequently opens it beak in order to obtain sufficient air, it is sleepy, torpid and exhausted. In such se- 
vere forms of the disease a large proportion of the affected birds die.

TrEATMENT.-The first indication in simple bronchitis is to place the affected birds in a comfortable, dry and reasonably warm place, where they will not be subjected to draughts of air, but where the ventilation is good. Give soft and cooling food, such as stale bread, or a mixture of bran! and middlings moistened with milk. Inhalation of steam, or vapor from boiling water in which hops or a small quantity of carbolic acid or creolin has been placed, is beneficial. Give with the food two grains of black antimony twice a day. A small quantity of flax seed steeped in hot water makes a demulcent drink that often gives great relief; and, in the absence of this, honey or gum arabic may be added to the drinking water with advantage. If the attack promises to be severe, it may sometimes be checked in the early stages by giving ten drops of spirits of turpentine in a teaspoonful of castor oil and repeating this dose after five or six hours. It should not be continued after there are signs of purging, for fear of exhausting the strength of the patient. In the very acute cases, where the whistling or snoring sounds with the respiration indicate a croupous form of inflammation, and where the gasping shows great obstruction of the air passage, relief may be obtained by giving from three to six drops of either the syrup or the wine of ipecac.

Medicines should be administered very carefully in diseases affecting the trachea and bronchia as otherwise they may enter the air passages and increase the irritation.

\section{CANKER.}

This is evidently a form of roup, and is also termed diphtheria in some forms of the disease. A cheesy substance forms in mouth and throat and often on end of windpipe. Remove the growth with sharp instrument and touch with piece of cotton dipped in permanganate of potash. There should be no part of flesh where growth has been untouched by the potash. In all cases where I use the knife I use a wash of peroxide of hydrogen and always wash instruments in this antiseptic. Treat the bird's blood with Epsom salts and sulphur. With young birds two applications will usually be sufficient, but older birds are harder to cure. Give cod liver oil in capsule if the bird falls off much in strength.

\section{GAPES.}

This disease is observed most frequently in young birds from one to four weeks old. The affected indivicluals are seen: to be less lively than usual; they extend the head fre- 
quently and widely open the beak-that is, they gape. If watched, they are seen to gape every minute or two, and as the disease progresses, this gaping is repeated several times a minute.

The gape worm is a small round worm, red in color, and both the male and female are found attached by their mouths to the mucous :membrane of the bird's trachea. The female is slightly more than one-half inch in length, while the male is only one-fifth inch.

They suck the blood of the bird, which forms their nourishment, and, in doing this, set up considerable irritation and even inflammation. The large and vigorous birds are not seriously disturbed by the presence of this parasite, particularly if the worms are not very numerous. Small and weak birds, however, are unable to successfully resist the combined effect of the loss of blood, the inflammation of the trachea, and the obstructed respiration. The strength is rapidly lost, and the windpipe becomes obstructed until finally the bird dies of exhaustion or asphyxia.

Separate the sick birds from the well ones as soon as the disease is detected and clean up the coops, pens and houses where they have been.

Disinfect all these places and also the drinking troughs or cups with a five per cent. solution of crude carbolic acid or with boiling water. Burn the bodies of the birds which die. Keep the infected birds in a house which can be easily cleaned and disinfected, and see that this house is kept disinfected with hot water or five per cent. carbolic acid solution. Megnin recommends for disinfecting enclosures and contaminated runs, sprinkling with a solution of one part of salicylic or sulphuric acid in one hundred of water. Provide pure water and uninfected food.

By careful manipulation the worms may often be removed from the trachea and the chicken radically cured in that way. To accomplish this either a feather or a long hair from the horse's tail may be used. If a feather is selected it is stripped of the web except near the tip. This may be used dry or it may be moistened with coal oil or oil of turpentine. If a horsehair is to be used it is folded and the two ends twisted together so as to leave a small'loop at the folded extremity. To extract the worms the chicken's mouth is forced open with the fingers of the left hand, and, when the glottis opens for breathing, the feather or hair is trust into the opening and downward into the trachea. It is then turned or twisted around several times and withdrawn, when one or more worms may be found adhering to it, or in other cases are coughed up, having been loosened by the feather or hair. 
These worms should all be burned as they are capable of spreading the infection. Great care should be used in these manipulations as the larynx and trachea are very tender and sensitive and are liable to serious injury from a broken or rough feather; or the bird may be suffocated by obstructing the breathing for too long a time.

Internal treatment is highly recommended by some authorities. Megnin had excellent results in treating affected pheasants with garlic. He made a mixture consisting of hard-boiled eggs, boiled beef's heart, the crumbs of stale bread and salad. These ingredients were chopped, poundecl and thoroughly mixed so as to make a paste. To this paste was added pounded garlic in the proportion of one bulb to ten pheasants each day, the garlic being thoroughly distributed through the paste. This mixture was greatly relished by the birds. Others who tried this remedy found that the birds refused to eat it, but that they would eat garlic in the form of a salad. Megnin also recommends assafoetida, seven and onehalf grains daily to a pheasant, used as a powder combined with an equal quantity of yellow pulverized gentian and mixed with the paste that was fed to the birds. He asserts that this treatment succeeded completely within a few days.

Some have obtained satisfactory results by administering camphor in the form of pills, the dose being one-half to one grain twice a rlay. Others have succeded with sulphur, giving one to three grains at a dose.

Those engaged in raising birds should depend rather on prevention than cure. The value of a thrce-weeks-old chicken is not sufficient to justify a great expenditure of time in its treatment. The effort should be made to place the young birds on uninfected ground, or the runs should be kept thoroughly disinfected. On the first appearance of the disease remove and isolate the affected birds and take the necessary precautions to secure the destruction of all the parasites they contain. By such measures the propagation of the worm will be prevented and the extension of the disease will be avoirled:

\section{PIP.}

Inflammation of the mouth is a rare rather than a common disease. The symptoms consist of the drying of the membrane of the mouth and particularly of that covering the tongue. A hard opaque deposit accumulates along the edges and upon the under surface of the tongue, and may firmly attach this organ to the adjacent parts. The dried epidermis may partly separate from the sensitive tissues, and form a hard shell which remains attached to the free extremity of the 
tongue, causing more or less painful constriction and interfering with the movements of the important organ.

This disease arises from any form of local irritation or injury which is sufficiently serious or long continued to set up inflammatory action. In the majority of cases it is probably caused by micro-organisms of feeble virulence which are able to make some headway when the digestive organs are deranged and the circulation of the parts somewhat disturbed. In most other cases, there is nothing more than a drying or desiccation of the mucous membrane caused by breathing through the mouth on account of obstruction of the air passages, or disease of the lungs affecting the respiration.

The common treatment which consists of tearing the dried epithelium off the tongue, before it has entirely separated by the natural process, is cruel and injurious, often leading to the death of the bird. In case of simple drying of the motth, it is sufficient to moisten the tongue with a few drops of a mixture of equal parts of glycerine and water. In case there is redness of the membrane, or if the epithelium is beginning to separate, or if a deposit has formed, add twenty grains of chlorate of potash to each ounce of this mixture. An excellent remedy for such cases is made by dissolving fifteen grains of boric acid in an ounce of water. Both of these solutions are harmless and may be freely and frequently applied. When the epithelium is separating, it should be kept moistened with the glycerine mixture and its detachment may be somewhat facilitated by loosening it with a pin or the point of a penknife, but great care should be exercised, the sensitive tissues should not be touched and no blood should be drawn. If properly treated the disease remains localized and is of slight importance. The exaggerated idea prevalent as to the dangerous character of "pip," probably arises from its being associated with serious diseases of the respiratory organs and from the fatal result which follow the forcible tearing away of the dried epithelium, leaving a bleeding and ulcerating surface. Should the drying and irritation of the membrane be the result of breathing through the mouth on account of disease of the air passages or lungs, the latter must, of course, be treated to effect a permanent cure.

\section{CROP BOUND.}

The affected bird has difficulty in swallowing or entirely loses its appetite. The crop is greatly distended, the swelling sometimes being of remarkable size. It is hard and more or less firm and unyielding on pressure. Sometimes a fermenting or decomposing liquid escapes from the mouth and nostrils. The bird is slow in its movements, dull, stupid, and 
sleepy. The comb is pale, the beak is kept open on account of the pressure on the trachea, and the feathers appear rough. In some instances sharp-pointed nails or other pointed objects are found to have penetrated the crop and skin and remain projecting from the puncture.

The pressure of the distented crop upon the wind-pipe and blood-vessels may cause asphyxia, the crop may become affected with gangrene, or it may even be ruptured. As much as three-fourths of a pound of moist or even sprouted grain has been removed from the crop in these severe cases.

The treatment of affected birds should be prompt and energetic to avoid the fatal complications which have been mentioned. Pour a small quantity of sweet oil into the mouth and cause the bird to swallow it. Then manipulate that portion of the crop nearest the throat by careful pressure and squeezing between the thumb and finger in such a manner as to break up the contents of the crop and force it towards the mouth in small portions. Suspend the bird head downwards from time to time and press the loosened particles of food towards the head so that they will escape from the motth. With care and patience the crop may be entirely emptied in this way, if oil is administered as often as is required to soften the contents. After this is accomplished give two grains of baking soda in water, keep without food for a day, and then feed sparingly on soft diet until recovery is complete.

In case the crop contains hard-pointed objects which cannot safely be manipulated in the manner just described, or if st'ch attempts at removing the impacted matter are for any reason unsuccessful, the crop must be cut into with a knife and the contents removed through the artificial opening. In order to perform this operation properly, the feathers should be clipped off with a pair of scissors over the most prominent part of the crop so that they will not obstruct the crtting or get in the opcrator's wy when he is removing the contents of the crop. Now take a sharp, clean knife and make an incision through the skin and the wall of the crop 'ntil the food in this organ is reached. This opening should not be much over an inch in length, and should be rather high on the crop so that the food will not have the same tendency to press it open when healing as it would at the most dependant part. The contents of the crop should be carefully removed with the finger, or with the handle of a spoon or some other convenient object. The wound may now be washed with a solution of carbolic acid, five drops to one ounce of water, and closed with stitches. White silk is the best material to use for this purdose. Some stitch the walls of the crop first, allowing the ends of the silk to hang outside, so that after five or six days the thread may be cut and the 
$\therefore$

stitches pulled out. The skin is drawn together in the same way. iOther operators prefer to stitch through the skin and the walls of the crop at the same time, drawing them carefully and neatly together. In five or six days if the union of the parts has taken place, cut the stitches and carefully draw them out so that they will not interfere with perfect healing. After the operation has been performed, give no food and only a small quantity of water, to which a grain or two of salicylic acid has been added, during the first twenty-four hours. Then feed with milk for two or three days, gradually changing to mush or more solid food, and finally return to the ordinary diet when the wound heals.

With fowls, this operation is not serious, if performed in time, the wound readily heals and the birds are soon well. With pigeons, it is usually fatal on account of the sensitive and vascular condition of the mucous membrane of the affected part.

\section{CONSTIPATION OR INTESTINAL OBSTRUCTION.}

Birds which are affected with serious constipation or intestinal obstruction become dull, lose their appetite, stand with arched back, roughened plumage, walk with more or less difficulty, are at times uneasy and make frequent attempts to expel the excrement. By careful examination, the hard impacted intestine may often be felt through the abdominal walls or the obstruction of the cloaca may be discovered. Frequently, the dried mass of excrement may be seen adliering to the feathers and blocking the external opening of the bowels.

When the obstruction has commenced and is maintained by accumulations of the excrement upon the feathers, the first step is to get rid of this obstructing mass. It should be soaked in warm water until softened, and then by clipping some of the feathers about the vent it is easily removed. If the cloaca is filled with a hardened mass, this too should be softened with warm water or with olive oil, and carefully manipulated until it can be pressed out or otherwise removed without injury to the bird. Oil may be inserted around such an obstruction with a small syringe, or, in the absence of a syringe, an oil can, such as is used for oiling machinery, answers the purpose tery well. After the oil has been applied freely, it may be necessary to wait an hour or two for it to penetrate and soften the obstruction before much progress can be made towards its removal by manipulation. The handle of a small spoon or a similar blunt instrument is often of assistance in cleaning out the contents of the cloaca.

When there is serious constipation or when obstruction has taken place high up in the intestine where it can not be reached by external aṕplications, laxative or purgative medicines are necessary. The most successful of these are Epsom salts twenty 
to thirty grains dissolved in a tablespoonful of water; castor oil one to two teaspoonfuls; or calomel one to two grains made into pills with butter or lard. The constipation should also be corrected as far as possible by regulating the rations of the birds. Give soft, watery mashes, green feed and bran until the bowels appear to be once more in a normal condition. The skillful feeder will generally prevent both diarrhea and constipation by careful attention to the diet.

\section{DISEASES OF THE LIVER.}

The liver is one of the largest and most important organs in the bird's body. It not only prepares the bile, which is one of the principal digestive liquids, but it assists in some of the most necessary chemical changes which occur in the blood. This organ contains numerous blood vessels through which passes a very large quantity of blood, and it is particularly subject to the attacks of various kinds of parasites. Most of these parasites probably find their way to the liver through the blood channels, lodge in the minute capillary vessels, and begin their multiplication and disease-producing action.

\section{CONGESTION OF THE LIVER.}

The liver of fowls is often found in a congested condition as the result of lack of exercise combined with over-feeding; also from the birds taking tainted or moldy food or poisonous substances of various kinds into their digestive organs; from the effects of infectious diseases, particularly cholera; and from obstruction to the circulation of blood by disease of the heart or lungs. It is often seen in birds which are in plethoric condition or very fat.

The symptoms are obscure and it is difficult to make a diagnosis during the life of the affected bird. The post-mortem examination reveals a greatly enlarged liver engorged with blood, tender and easily torn or crushed.

If the condition is suspected in time for treatment give sulphate of magnesium or sulphate of sodium in a purgative dose (20 grains to a dram) and follow with sulphate of magnesium ro grains, bicarbonate of sodium 2 grains, repeated daily for a week.

The disease should be prevented by proper feeding, regular exercise, and protection from parasites and infectious diseases.

\section{INFLAMMATION OF THE LIVER, HEPATITIS.}

This disease is due to the causes enumerated as producing congestion of the liver and is a different and more advanced stage of the same process. 
The symptoms are loss of appetite, sluggishness, tenderness over the abdomen and sometimes a yellowish color or jaundice of the skin.

The treatment of birds so affected is not profitable unless they are very valuable for breeding or exhibition purposes. It is well to begin with $1 / 2$ to I grain of calomel, followed with 20 grains of Epsom salts and 2 grains of bicarbonate of soda after twelve hours. Naphthol or benzonaphthol may be given twice a day in I grain doses to disinfect the intestinal canal. If the diarrhea is excessive and weakens the bird, treat as recommended for that disease.

When marked improvement is shown, give green food, tonics, and raw beef, and allow the bird free exercise in the open air.

\section{DIFFICULT LAYING, EGG BOUND, OBSTRUCTION OF THE OVIDUCT.}

Laying may become difficult because of irritation of the lower part of the oviduct arresting the secretion of mucus and causing the mucous membrane to become dry and lacking in its normal lubrication. The same trouble results from birds being too fat or from the eggs being too large, and is aggravated by constipation. If the cause is sufficiently pronounced in its action, the affected bird may be unable to expel the egg; this is arrested in the passage where it sets up inflammation and leads to straining and possibly to prolapsus of the oviduct.

Symptoms.-When fowls are egg bound they at first go frequently to the nest, making efforts to lay, but are unable to accomplish this function. They are restless and evidently in more or less distress. Later they become dull, with rough plumage and are indisposed to move. On examining the bird by pressure of the finger about the vent, the egg can be distinguished as a hard body in the posterior part of the abdominal cavity. In case of prolapsus, the everted oviduct may be easily seen.

TREATMENT.-In the early stages when the irritation is slight, it is sufficient to inject a small quantity of olive oil and gently manipulate the parts. Afterwards, give cooling food, and, if hens are too fat, reduce the ration. In case the expulsion of the egg can not be obtained by the injection of oil, immerse the vent and lower part of the body in water, as warm as can be used without injury, and hold it there half an hour or more until the parts are relaxed. Then inject oil and endeavor to assist the bird by caref 11 rressure and manipulation, or by dilation of the passage. Fluid extract of ergot is useful in these cases and may be given in five-drop doses three times a day. In 
some cases it is necessary to puncture the egg, allow the contents to escape, crush the shell and remove it in pieces.

\section{CONGESTION OF THE BRAIN, VERTIGO. - $\cdots$}

Congestion of the brain is a disease quite frequently seen with poultry. It may occur in fat and plethoric birds by fright or indigestion. It is frequently associated with irritation of the intestines by parasitic worms. It is seen particularly in male birds during the breeding season and in chickens exposed to the hot rays of the sun. It may, also, occur as the result of blows upon the head and as a complication with infectious diseases such as cholera.

The symptoms are giddiness which leads the bird to throw its head upwards and backwards or to bend the neck to one side. The gait is staggering and uncertain, the bird walking backwards, sidewise or in a circle. Sometimes irregular and unusual movements are made, or the bird falls to the ground fluttering and making convulsive movements with the legs, or may lie for some minutes powerless to move. In some cases there are epileptiform spasms which may often be stopped by a touch, in other cases there is great stupefaction and drowsiness.

As treatment apply cold water or ice to the head and hold it in position until the head is thoroughly cooled. Give internally 30 grains of Epsom salts, or one and one-half grains of calomel, or two teaspoonfuls of castor oil. Keep the affected bird in a quiet, cool, and shady place. If there is not entire recovery under this treatment, try bromide of potassium one to five grains three times a day dissolved in a tablespoonful of water. In case of intestinal parasites endeavor to dislodge these with appropriate remedies.

\section{HEMORRHAGE OF THE BRAIN, APOPLEXY.}

Apoplexy is the result of a rupture of one of the blood vessels of the brain and pressure from the escaped blood. Such accidents are attributed to stimulating food, to overfeeding and mechanical injuries, to violent exertion and to straining in laying eggs (hens being sometimes being found dead on the nest from this cause).

There are no premonitory symptoms in this disease. The bird is attacked suddenly, falls nearly or quite insensible, or dies upon the nest.

In most cases treatment is impossible as the bird dies before it can be administered. In mild attacks the treatment should be the same as recommended for congestion of the brain. As preventative measures, regulate the rations and give plenty of exercise. 


\section{CHICKEN POX.}

This disease chiefly affects the head of poultry, and appears as an eruption of round or oblong, yellow nodules, varying from the size of a pinhead to that of a pea or a grain of corn. The largest are found about the beak, the nostrils, the eyelids, and upon other parts of the head that are free from feathers. They form rough, yellow masses upon the comb and wattles. The eruption may be localized where it first appears or it may extend to the feathered portions of the body.

With pigeons the eruption has a tendency to become diffuse and invades the neck, the lower surface of the body, the inner sides of the legs and wings, the rump and even the upper surface of the wings.

The nodules, which at first have somewhat the appearance of the warts often seen upon the hands of children, reach their full development in from five to ten days. They soon show a central depression, an opening forms, or the summit is rubbed off accidentally or by the bird's beak, after which there is a discharge of watery, or, later, of a thick, yellowish matter which soils the feathers and which if abundant may become foul and disagreeable.

When the disease is localized, the general health of the bird does not appear to suffer, and spontaneous recovery may occur. In this case the nodules dry up and form a crust and fall off either as a whole or in particles. Generally, however, the disease extends, the birds become emaciated, the plumage is rough, the strength is exhausted, and death results. In the most violent cases, especially with pigeons, the eruption extends to the mucous membranes of the mouth and nostrils, the resulting inflammation takes on a diphtheritic form, and early death follows.

The prophylactic or preventive treatment consists in excluding affected birds from the premises; in keeping the poultry-houses and pigeon-cotes clean and dry; and in hatching the chickens early on farms where the disease is troublesome. Disinfection by whitewashing the houses, or spraying them thoroughly with a solution containing three to five per cent. of carbolic acid and then opening them for the drying action of the sun and air is a valuable means of guarding against an outbreak of the disease.

The curative treatment consists of the local application of those remedies which have been found efficient for the destruction of the parasitic blastomycetes. Some person's have been successful by feeding sulphur, and at the same time, applying sulphur ointment twice a day to the nodules. Others have cured the affected birds by applying carbolic ointment, 
or glycerine containing two per cent. of carbolic acid. Another treatment is to bathe the affected parts with soap and water in order to soften the crusts, and afterwards apply a solution of sulphate of copper (bluestone), a dram to $1 / 2$ pint of water. Sulphate of copper has been found destructive to many kinds of fungi, and, therefore, is promising in this disease. Tincture of iodine has also been recommended, both by itself and mixed with ten per cent. of carbolic acid, but this remedy is rather severe and should only be applied sparingly and after the others mentioned have proved ineffectual.

This local treatment should be accompanied by cleaning and disinfection of the houses, and, if possible, changing to new, uninfected runs. The feeding troughs and drinking vessels should be daily washed with boiling water.

When the disease first appears in a flock the affected birds should be at once isolated and treated, and the effort made by disinfection to prevent the further extension of the contagion.

\section{RHEUMATISM AND GOUT.}

All species of fowls and especially chickens are subject to rheumatic affections. The trouble begins with spasmodic jerking of the legs, and is followed by lameness, indisposition to remain standing, painful joints and refusal to walk. The feet and next joint above (tibio-tarsal), are most frequently affected. After a time small swellings appear on the sides of these joints, which are at first soft, and somewliat painful, but gradually become more firm until they feel like enlargements of the bones. Sometimes these swellings ulcerate, in which case the sores appear red, irregular in outline and bleeding. A yellowish, flaky or stringy pus partly fills the sore and when this is removed the naked tendons, bones or joints may sometimes be seen. The cavities of the joints are opened to the air, fistulas develop and death of the bone occurs. The disease is chronic in its development and the advanced stages are, therefore, generally seen in old birds. The affected birds may live a long time even when the disease has progressed to the extreme degree described above. They lose flesh, however, their plumage becomes rough and dull, diarrhea sets in and death occurs from exhaustion.

TREATMENT.-As rheumatism is usually caused by exposure to cold and dampness, the development of the disease in one or more fowls of a flock should be a warning to improve the ventilation and drainage of the houses. The buildings where the fowls live and roost should be thoroughly dry, free from draughts, yet well ventilated, and not too cold. IVith proper attention to these sanitary arrangements the clisease should 
be prevented. Affected birds should have frequent change of ration with plenty of green feed. Begin treatment with a dose of Epsom salts, 20 to 30 grains. The following day add 30 to 40 grains bicarbonate of soda (baking soda) to the quart of drinking water, and give 2 to 3 grains of salicylic acid twice a day. Apply camphorated or carbolic ointment to the affected joints. The birds which are seriously affected would be better killed than treated, and the main reliance must be under any circmstances in prevention by securing proper sanitary conditions.

\section{CORNS.}

The condition known as corns is an inflammation and thickening of the skin on the under surface of the foot, the result of prolonged pressure, irritation, and bruises. Corns are generally caused by too small or too narrow perches which compel the fowls to grasp them tightly in order to maintain their position. This firm grasp continted night after night affects the circulation of the part of the foot that comes in closest contact with the perch. More or less irritation and inflammation is set up, which leads to multiplication and enlargement of the cells of the part, and results in swelling and thickening of the skin. A similar condition may be caused by heavy birds flying from their perches and alighting upon a stony surface or hard floor.

Suitable precautions should be adopted to prevent the development of corns. The perches should be broad and flat, they should be of a proper height, and the floor upon which the birds alight should be covered with earth or sand so the feet will not be bruised. The affected birds should be treated by paring off the thickened epidermis, without causing bleeding, and applying boric acid ointment (boric acid I part, vaseline 5 parts), or painting the part with tincture of iodine.

\section{BUMBLEFOOT.}

This is an aggravation of the condition known as corns. It is seen in those cases where the irritation and bruising is most severe or prolonged. The affected part is hot, painful, and more or less swollen. Sometimes an abscess has formed, or in older cases this abscess may have broken and left a suppurating sore. In the most severe cases the joints may be inflamed, and may even be penetrated by the pus channels which have formed from the abscesses.

As treatment employ the preventive measures already mentioned for corns and other injuries to the feet. Soak the feet in warm water for a half hour twice a day and poultice 
until the inflammation is reduced. If an abscess in the sole is indicated by a soft, fluctuating swelling of the part, it should be opened with a sharp knife. After the poulticing is completed apply boric acid ointment and protect the feet with a piece of cotton cloth. If the joints have become affected, and particularly if these communicate with the pus channels and are the seat of suppuration, the bird should be killed, as its treatment would be unprofitable, anst its entire recovery would not be probable.

\section{SCALEY LEG.}

Scaly legs is a form of scabies or mange caused by the mite known as the Sarcoptes mutans. It is a strictly contagious disease although it does not spread rapidly from tird to bird, and there may be only a few noticeably affected birds in the flock. The Asiatic breeds are most susceptible to it, and many birds, even of these breeds, resist the attacks of the mites, and never show any symptoms. It attacks quite a variety of birds-fowls, turkeys, pheasants, partridges and cage birds, but has not been observed in ducks and geese. In the production of scaly legs, the Sarcoptes mutans penetrates beneath the epidermic scales on the upper surface of the foot and the front of the shank, and by burrowing there sets up an irritation which leads to a multiplication of the cells of the part, and an exudation of serum. It is by the union of these two products that the white, powdery crust is formed, which raises the epidermic scales from their normal position.

When this disease is first observed in a flock, prompt and energetic measures should be adopted to eradicate it. The affected birds should be isolated to prevent the spread of the contagion. The houses which they have occupied should be thoroughly cleaned. The roosts and other, woodwork should be scalded with boiling water or covered with carbolated lime wash. The treatment of the affected birds is the same as with scabies of the body, with the single exception that the loosened scales must be removed in order to bring the remedy in contact with the mites. In order to accomplish this the legs should be soaked for a suffcient time in warm water to which some soap has been added. When thoroughly softened the loose scales may be removed without causing bleeding. Or, a coating of soft soap may be applied to the affected parts, leaving it to act for from twelve to twenty-four hours. The legs are then placed in warm water and the scales softened and removed by gentle rubbing and friction. After this has been done the legs are dried and treated with a good coat of Helmerich's ointment, Creolin ointment ( $\mathrm{I}$ to IO), Carbolic ointment ( $\mathrm{I}$ to $\mathrm{ro}$ ), or bal- 
sam of Peru. The later may be applied daily for three or trur days, and is better adapted than the other remedies to the treatment of young or delicate birds, since it is equally efficacious in destroying the mites and has little if any injurious effect upon the birds. The disease is not a difficult one to cure, if the preliminary treatment is thorough and the loose scales and crusts are all removed.

\section{TUBERCULOSIS.}

The symptoms which are observed in the tuberculosis of birds are common to other diseases, and, therefore, while they are valuable as an indication of the nature of the disease, they are not sufficient to permit an absolutely reliable conclusion to be reached. There is rapid and progressive emaciation, made apparent by the loss of weight, wasting of the muscles, and prominence of the bones. The comb becomes pale, the bird loses its bright, animated expression, and in the later stages becomes quite dull and sleepy. At this time a persistent diarrhea appears, which increases in intensity until the subject dies from exhaustion.

The symptoms just enumerated are those observed when the disease is confined to the internal organs. It often.affects the joints and bones, when it is revealed by lameness, swellings of the joints and deformities of the bones. Occasionally ulcers form, in the pus of which many bacilli are found.

The tuberculosis of birds, like that of mankind, is caused by a bacillus. The bacillus of birds in a general way resembles that of man, but is larger, more vigorous and hardy, and grows better outside of the body. The disease is strictly contagious, and usually appears in a flock as a consequence of bringing in new birds. That is, it results from contagion carried by birds from other diseased flocks.

The eradication of tuberculosis in birds from an infected premises can only be attempted with a fair prospect of success when all the birds are sacrificed. Any individuals that are preserved are liable to have ulcerations of the intestines, from which the bacilli are constantly distributed. There should, consequently, be no attempt to save any birds from an infected flock.

When the birds are all killed and disposed of by burning or deeply burying, the premises should be carefully disinfected. The manure should be carefully scraped and swept together and saturated with a 5 per cent. solution of carbolic acid or mixed with lime. The floors and woodwork of the houses should be washed with boiling water or with a hot solution of carbolic acid. The feeding troughs, drinking vessels and nests should be treated in the same manner. Any 
yards used for penning birds should be sprinkled with the carbolic acid solution.

After the cleaning and disinfection is accomplished the premises should be opened to the sun and air for a month if possible before new birds are introduced. It is then a good plan to cover the walls and roosts with lime wash, to which 4 ounces of crude carbolic acid to the gallon has been added, and ventilate a few days longer before the houses are used. Those who scrupulously enforce these measures may be reasonably certain that the contagion will be destroyed and that the new flock may be safely introduced.

\section{INJURIOUS HABITS OR VICES.}

\section{EGG EATING}

One of the most troublesome and injurious of the acquired habits of poultry is cgg eating. Undoubtedly the habit begins in most cases by the accidental breaking of an egg in the nest, but it rapidly spreads among the fluck until a large proportion of the egrss laid are purposely brolien and caten by the hens. The heary breeds of fowls are most subject to this habit because they more frequently break their eggs by stepping upon them than do lighter birds. When an egg is broken it is ton tempting a morsel to be left in the nest. The hen not only eats it, but often carries large pieces of the shell al,out the house or grounds, pursued by other members of the flock, each of which wants a portion. In this manner a number of individuals soon learn how appetizing are eggs and egg shells, and each in turn becomes a teacher of others.

It is plain that whatever conditions contribute to the breaking of eggs in the nest may be considered as causes of the habit. Thin-shell eggs are easily broken, and, hence, a deficiency of shell-forming constituents in the ration may be a cause. In other cases an egg may be broken for want of sufficient straw in the nest to protect it from direct contact with the wood.

To guard against the formation of the egg-eating habit the fowls should have plenty of lime, oyster-shells, bone, or similar substances to ensure a firm shell upon the eggs. The nests should be properly supplied with straw, and artificial nest eggs should be used. In this way the danger of breaking eggs is reduced to a minimum. It is, also, well to have the nests rather dark so that if an egg is accidently broken the hen will not discover it.

When the habit has been once acquired, these precautions are not always sufficient, and it may be necessary to construct the nests so that the eggs will roll beyond the reach of the hen. Some people place artificial eggs in the nest and about the houses so that the hens may peck at them and get the idea that they are no longer able to break egg shells. Others blow out the 
contents of a few eggs through a small hole in the shell and fill the space with a paste consisting largely of mustard, capsicum, alves or other disagreeable compounds and leave these where the hens will find them.

Under any circumstances, it is best to remove the egg eaters from the remainder of the flock, and, unless they are very valuable, to kill them for the table. If they are too valuable for this treatment, then dark nests or those in which the egg rolls into a safe receptacle are the only resources.

FEATHER EATING, FEATHER PULLING.

Fowls often acquire the habit of pulling out and eating their own feathers or the feathers of other members of the flock. This vice is acquired most frequently in the Spring or at moulting time. The first indication that the birds are pulling feathers is the loss of plumage, bareness and redness of the skin over the posterior part of the back. Sometimes the skin has become abraded by repeated pecking and sores result. If the hirds are watched carefully, they will be seen to catch one or more feathers in their beaks, tear them out and swallow them. They are apparently most fond of the young, growing feathers, the quills of which are filled with blood.

Various causes have been assigned for this habit, the most probable being improper rations and insufficient exercise or idleness. In some instances, it may originate from irritation of the skin caused by animal parasites. In general, it may be said that anything which produces an abnormal appetite predisposes to feather eating.

As preventive measures, the birds should be given a free run or they should at least be made to scratch for a large part of their food. This exercise improves their digestion and gives them something to occupy their time. As in some cases, the habit is due to insufficient animal matter in the ration or to feeding too long on a single kind of grain, particularly corn, one of the first measures adopted should be a well balanced ration containing skim milk, meat, bone, vegetables or green feed and frequently varied.

The skin and feathers should be carefully examined for lice and mites, and if these are found the remedies recommended for such parasites should be applied. As lack of grit may be the cause of indigestion, this essential element of the ration should receive attention, and it is well to give 6 or 8 grains daily of a mixture of equal parts of powdered gentian root and ginger. The birds should also be examined for intestinal parasites. 


\section{Starting a Poultry Ranch on Small Capital.}

One of the questions asked by visitors on a poultry ranch is, . How much does it cost to start a little chicken business and how can I get started in my back yard on a fiity-foot lot?" When we tell you that we have successfully hatched and raised several thousand on an acre, and there are only four or five city lots to the acre, you can see that considerable can be accomplished in your back yard. Begin by engaging from some reliable breeder, 50, 100, 200, 300 or 500 eggs. We say "Begin by engaging eggs," because you will have to place your order and wait your turn for them. Then order your incubator, set up and tested by the seller. Next you will require brooders. A couple of days in the incubator after the hatch is best for little chicks before transferring them into the brooder. The little birds can be kept in the outdoor brooder about a month, three weeks with heat and one without heat; then they are ready for the pens in which cold brooders should be provided under shelter. Our cold brooders are home-made affairs, nothing more than boxes five feet long, two wide and two feet high, with movable floor made of laths to form little perches. The front is open except for a burlap curtain, which we throw back day times and drops down nights. The little birds will roost in these cold brooders until they get a couple of months old, when they will begin roosting on top of them and on regular roosts, if provided. Now, regarding expense; thus far your eggs have cost you from $\$ 5$ to $\$ 30$ a hundred, according to quality, your incubator $\$$ Io to $\$ 42$, according to size and make. Out-door brooders cost \$12 to $\$ 16$ each per roo-chick size. To make a calculation of cost, we will figure on the large incubator and medium-priced eggs, and it comes out as follows: Three hundred and sixty eggs at say $\$ 15$ a hundred, $\$ 54 ; 360$-egg incubator, $\$ 42$; three outdoor brooders at $\$ I 6, \$ 48$; oil for incubator 22 days at $I^{1} / 2$ cents a day, 33 cents; oil for three brooders, 22 days at $1 \frac{1}{2}$ cents, 99 cents; 20 pounds chick feed at 5 cents, \$I. Total, \$I 46.32 . Three weeks for birds to hatch, and three weeks in hot outloor brooder, and you should have about 270 strong birds, three weeks old. This is a conservative estimate, allowing for a loss of 25 per cent. which is a safe calculation if careful attention is given! and eggs purchased on a IOO per cent. grarantee. At this stage you are well equipped for business, except your pens and roost-houses, which will be needed when 
your birds are about three months old. We will give yout a tested working plan for a little home place such as you see on every street in Los Angeles. Say your lot is 50xi50, with the back yard fenced in. Your contemplated pens are to be eight feet wide and forty feet long, and you may want to add a number of similar pens from time to time, as your flock increases. With this thought in view you build your first pen lengthwise against the back fence of your lot, which is 50 feet. Your pen being only 40 feet, leaves Io feet in front of the pen to form an alley. When other pens are needed they can be added in the same manner, only requiring front and near side as back and far side is already there. In the back end of pen you build a little house $8 \times 8$ feet of cheap, second-hand or resawed lumber. The back and two sides of said little house should be lined with paper three or four feet up from the ground to insure against draughts. The front should be two burlap doors about $4 \times 8$ each. The best roof is galvanized corrugated iron, and cheapest in the long run, but shingles, shakes or tar paper can be used. The cost of such a pen and house is as follows: Four posts, $3 \times 4 \times 8$, set Io feet apart on long side, and one post extra to hang front gate on, cost \$I. A solid piece of lumber should be nailed along the ground from post to post, and can be of any desired dimensions, but we use a IXIO, cost 80 cents. The lumber in the house amounts to but little, as the back fence forms the far wall and the side fence the rear wall, consequently you have only the rear wall and the two burlap doors to build. The wall is $6 \times 8$ feet, 48 square feet of lumber at $2 \mathrm{I} / 2$ cents, or $\$ 1.20$. The burlap door frames can be made of Ix3 lumber covered with two-inch wire netting and barley sacks, all material costing less than $\$ \mathrm{I}$. Then you want 48 running feet of 2 -inch wire netting 5 feet high, to run around the near side and front of pen; at half a cent a square foot would be \$1.20. Roof of galvanized iron 9x9, or 8I square feet, at 4 cents, or $\$ 3.24$. One hundred running feet of $2 \times 3$ for studding and roof timbers will be more than enough, and cost \$1.25. Labor for such a house and pen: costs me about $\$ 2$, but make sure by calling it $\$ 4$, and if you allow, say, \$I.3I for nails, hinges and extras, you have a safe estimate of $\$$ I5 for each house and pen. You should select the best pullets from your six-months-old birds and sell the rest for market stock, possibly getting a better price for a number of the cockerels, if they are suitable for hreeding. With this money purchase the necessary number of mature breeding cocks, and when your pullets are fully matured and laying well, mate up your pens. Of course you will, before mating time, have finished up the number you will need. Your market stock should 
sell for I 5 to 35 cents a pound, live weight. A good breeding cockerel will sell from $\$ 5$ to $\$ 50$, but of course the $\$ 50$ birds are few and far between, and one usually has to breed for special points to get that price. Now the egg orders should be looked for, and a small "ad" in a good poultry magazine and a local newspaper will attend to that matter. You can probably run many hatches the second year, as there is great demand for newly hatched chicks, and many people raise very few of the number hatched, selling most of them, and make a very good thing of the baby chicks. We know of one woman who hatched I8oo, saved 7oo for her next year's breeding yards, and sold IIOo at good prices. Of course a backyard chicken plant is but an introduction to acreage property and another prosperous chicken ranich.

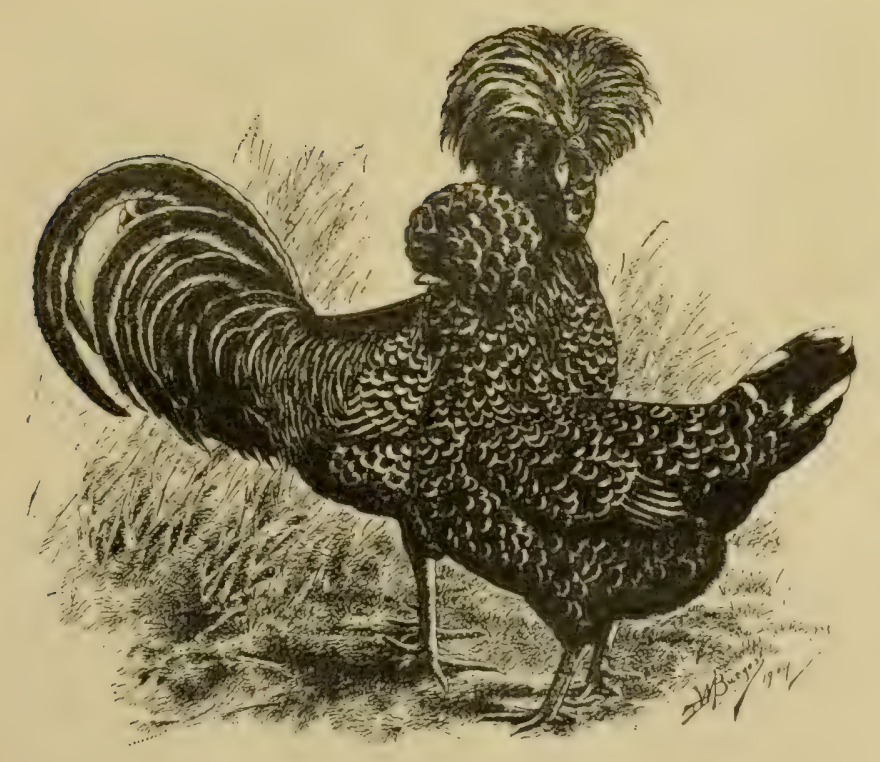




\section{A Beginner's Experience.}

There are numerous ways of making the poultry business pay handsomely, in fact it is getting to a point where one must specialize. One person is peculiarly adapted to mating and breeding for points, another is not at all interested in that line and will make a successful egg rancher or run to the various kinds of market poultry, broilers or capons, etc. I have in mind a little woman whose talents ran in the firstnamed direction. She purchased a pen! of six laying hens and a cock of popular variety, a very fine pen. She lived in a little cottage on a 50xiso foot lot. One thing which she prized next to her birds was a thickly-sown white clover lawn I $5 \times 50$ in the front and IOx 50 on the side. Her cottage and a little back porch occupied a space $40 \times 60$, leaving a space $75 \times 50$ feet in the back for her yards. She made a good roosting shed out of a piano box, nest boxes out of soap and tomato boxes which her grocer brought her. She made plenty of shade by stretching gunny sacking over four posts driven into the ground and with a couple of fruit trees kept her birds comfortable and their plumage richer by keeping the direct rays of the sun off. I know most of you are thinking, "Why didn't she have better roosting quarters and nests," but this was entirely a pin money affair to start. She filled small cigar boxes with grit, shell, charcoal, and beef scraps and nailed them to the fence so the birds could easily reach them with the bill and not the feet. They were always supplied with plenty of fresh, cool water, green clover clippings, and kept free from lice, and being grateful hens they began to pay her for their comforts with plenty of fine-shelled, fertile eggs which any good hen will do if given the necessary ingredients for their manufacture. Our friend read all the poultry literature she could beg or borrow and took two good poultry magazines. In ten days she had two settings of thirty eggs which she set under two cheap mothers which she purchased from a neighbor.

Her birds were set on the ground dampened and hollowed out to a nice, round nest lined with hay. A generous handful of lice powder was sprinkled in the nest and the aforesaid motherly hens were carefully deposited on a few china eggs. After they had demonstrated their desire to make a stay of 
it the china eggs were exchanged for the fine eggs and they settled down to business.

A fence of chicken wire separated them from the other birds and two nice little individual pens were fixed up with a dust bath in one corner, fresh water, a dish of corn and wheat, and one of grit was always before them. The droppings were cleaned up each morning, the hens dusted a couple of times during each hatch, and with such treatment they brought off twenty-eight fluffy little babes who immediately started in to scratch and grow as these birds can with half a chance. The chicks were all given to one hen, not fed for thirty-six hours and then were given grit for a first meal, followed by a judicious mixture of small grains; after three days of this a dish of beef scrap and one of chick feed was placed before them and they were on the high road to big chickendom. They were of course, carefully greased on the tops of their little heads and under the neck on being allowed their first meal and the mother carefully dusted again. Their nest was changed, the mother kept within the little enclosure and the babes allowed to run. They do not go away far when the mother is kept shut up and grow so much better than when the mother is allowed to run also.

The same care was taken of the next little brood and soon the chicken yard, which by this time had been divided into six pens $12 \times 50$ ft. each, was filled with the small birds. She started her first hatch January I5, and every succeeding Ist and I5th saw two hens set and carefully cared for until July 22nd saw the last hatch. There were at this time twelve hens with their broods. The first hatches beat the late ones in size but the color ran better late in the season. Each one of these twelve hens had two broods to. raise. August I 5 th the first four hatches were divided as to sex, and the cockerels that ran at all to legginess, red in plumage or poor combs were fattened and sold at 25 cents a pound to the butcher. The pullets were all kept. By September I5th there were I75 good birds on the place after a second culling. She picked out the best cockerel for herself and fifty of the best pullets. 'The remainder of the pullets were put in a neighbor's yard which she rented for a very small sum and divided into three pens; in one she put the twenty-four hens, her incubators for that season which were replaced by mechanical ones for the next. In the other two pens she put the sales cockerels and pullets and placed an ad. in one of the poultry magazines and the largest daily paper. One of the cockerels was purchased by the breeding farm from which she got her original stock, for $\$ 25$, and she sold several for $\$ 5$ and $\$ 10$ and all her pullets at $\$ 2: 50$ to $\$ 15$.

Don't forget that she had purchased the best stock she could obtain and knew they had been carefully bred from good stock 
many generations back. Her success would not have been so great had she not been able to show to the customer an unusually good bird.

She cleared above her feed bill and other expenses, $\$ 600$, besides increasing her stock that first year. The next year she took the old male bird and mated him with twelve of the best pullets, took the finest of the cockerels and mated him to the six hens; there were two fine breeding pens. She exchanged two fine young cockerels for two cock birds with the ranch that supplied her first stock, and mated up two more pens. Now she was cramped for room, so sold to this ranch any number of eggs, as well as to other customers, for the customer of the year before always comes back again after fair treatment.

Her husband about this time woke up and purchased a tenacre ranch in a good location and this small beginning bids fair to be one of the best ranches in the state. The ranch with which she had had such pleasant dealings, farmed out hundreds of their chickens to her and sent any number of customers to her that they could not supply, each being of great benefit to the other. There is so much to be made out of poultry in this Western country that every one in the business should encourage others to go into it. There is such a demand for poultry of all kinds, the finely bred, market and egg-producing solely, that too many ranches can not be established. Of course there is plenty of work in poultry raising and I think that is one of the reasons that so many failures are made. People think there is nothing to it but to purchase a lot of birds and let them do the rest on feed whenever the spirit moves the owner to supply it. They should be fed regularly, housed in quarters where no draft can reach them, but plenty of good fresh air; no vermin should be tolerated a minute and no filth of any sort. It would take a fine tooth comb to get any filth from a modern, up-to-date ranch that is paying well, but when you visit a place where your nostrils are offended and the chicks are droopy, the water foul, and roost-houses sunless and filthy, just say to yourself, "No money is being made here." In all probability the owner will tell you so himself and try to discourage the prospective chicken rancher, but did you ever have a clean, wellbuilt, well-stocked, big paying ranch offered to you? If you did and didn't take it, write to me about it and I can sell it to any one of a dozen people anxious to get established. 


\title{
Questions and Answers.
}

\author{
CALIFIORNIA ADVANTAGES.
}

QUESTION-What are the advantages in California, over other States, as regards poultry raising?

ANSWER-A mild climate especially adapted to poultry culture-the cheapest of buildings necessary for a poultry plant-one of the best markets in the world, with unlimited demand--land of any description and price-the advantages of combining poultry with fruit, bees, alfalfa or other agricultural pursuits.

DOUGLAS MEIXTURE.

QUESTION-What is Douglas Mixture and how is it made?

ANSIVER-Douglas Mixture is a good tonic, and is made as follows: Sulphuric acid one half-ounce and copperas one-half pound in a half gallon of water. It is administered by placing a tablespoonful in the drinking water for a dozen fowls daily.

\section{BRASSY PLUMAGE.}

QUESTION-What causes brassy color in white fowls? Does the feeding of yellow corn have a tendency to increase or maintain the brassy and cream color in white fowls?

ANSWER-Brassy color is inherited from the parent stock just as any other color defect, but is often brought out and intensified by the rays of the sun. There are many who think that yellow corn fed to white birds affects their color, but it has never been satisfactorily proven.

\section{OVERFED.}

QUESTION-I have about sixteen Plymouth Rock hens, have kept them housed up all winter. Some of them weigh nine to nine and a half pounds, but they do not lay any eggs, and seem to want to loaf about and do nothing. What is the cause of this?

Ansiver-The hens have been fed to death on fattening food of some kind. Put the hens to work, bury all their food in with the grain, and spade the dirt over it, so that they will have to dig and scratch for every bit of food they get to eat. If this plan is followed out, the hens will do good service.

\section{LICE AND MITES.}

QUESTION-Now that we are overrun with work, the lice and mites have gained a considerable foothold in all the cracks and crevices of our poultry house. Under the roosts are what look like bunches of swarming bees. The mites are so thick and 
so determined that they almost eat up the hens. What shall I do?

ANSWER-Proceed first to sweep down the ceiling and the side walls of the entire interior of the house thoroughly with a stiff broom, and destroy every particle of hay or straw from the nest boxes. Sweep and clean the dropping boards and the floors of the henhouse thoroughly, and remove every bit of the litter that has been accumulated in this way to some excluded spot, and burn it. Spray every crack and crevice of the poultry house, including nest boxes, with kerosene oil. Spray the nest boxes, the roosts and the dropping board with a good covering of some good liquid lice killer. Dust all birds with a good insect powder until your fowls are free from vermin.

\section{SCORE OF AN EXHIBITION PEN,}

QUestion--How is the score of a pen of birds in the show room determined?

ANSWER-An exhibition pen consists of four females and one male. The scores of the four best females are added and this total divided by four. To the amount thus obtained is added the score of the male, and this total is score of the pen.

\section{PRESERVING EGGS BY THE WATER GLASS METTHOD.}

QUESTION-What is the best method of preserving eggs?

ANSWER-Careful tests have been made with various compounds, and Water Glass has in each case proven most satisfactory. It is also called Soluble Glass. The chemical name is silicate of sodium or silicate of potassium. It can be purchased at any drug store, and costs about 50 cents per gallon, which is sufficient to preserve 50 dozen eggs. To one quart of Water Glass add ten quarts of water. It is best to use water that has been boiled and is perfectly clean. The eggs should be wiped clean, but should not be washed. Earthenware jars are best for storing the eggs, which should be entirely submerged in the liquid. If wooden barrels or kegs are used, see that they are first thoroughly scalded with boiling water. Eggs can be kept by this method from nine to twelve months.

HOME MADE LICE POWDER.

QUESTION-How can we make good lice powder to dust our birds with. We use dusting powder in large quantities and find most of the prepared powders very expensive?

ANSWER-We have found the following a very effective dusting powder and very cheap to use where the flock is large. The powder is made as follows: 50 lbs. sulphur, Io lbs. sifted air slacked lime, 25 lbs. sifted road dest, 5 lbs. Buach insect powder. Mix thoroughly and hold fowl over a barrel while dusting. 


\section{CULLS.}

QUESTION-What is meant by "culls"?

ANSWER-The term "cull" is given to fowls that are from the best breeding, but are lacking in strong development of one or more features which must be present to make them good exhibition specimens.

\section{ALFALFA AS A POULTRY FOOD.}

QUistion-Is alfalfa a good poultry food? What is the best method of feeding it? When and how should it be planted?

ANSWER-Alfalfa is the ideal green food for poultry and will supply about one-fourth of the necessary food supply. It can be cut and run through a clover cutter and fed in troughs or the birds can be turned into the growing alfalfa at regular intervals; they will kill out the crop if too many are allowed on a limited space. Plant just before the last winter rains after the possibility of frost killing the young growth is past. Have the ground carefully prepared and plant the seed so that it is just covered with fine earth.

\section{DRY FEEDING AND MASH.}

QUESTION-What is a good mixture of dry grains for dry feeding? What is the best system for feeding under this system? Can the ground grain mixture be used as a wet mash when desired?

ANSWER-Mix IOo lbs. bran, 50 lbs. shorts, 50 lbs. corn meal and $2.5 \mathrm{lbs}$. beef scraps. Feed dry in troughs, leaving the feed before the birds until they eat their fill each morning and remove what is left. Give cut alfalfa at noon and a mixture of whole wheat, barley and oats and kaffir corn, scattered in scratching material, for the night feed. The mixture given above for the dry morning feed is equally valuable when used as a wet mash.

\section{PRICES OF GRAINS.}

QUESTIONS-At what price can grains for feeding poultry be bought for in California markets?

ANSWER-The prices vary on the different grains at different points in the state, as in the case of wheat; in the great wheat sections this grain can be bought cheaper than in other parts of the state. From quotations at hand we can give the average price charged for the following grains during the past five years: Barley $\$ \mathrm{I} .20$ per Ioo lbs; Wheat $\$ \mathrm{I} .70$ per 100 lbs.; Kaffir Corn \$r.65 per Ioo lbs.; Corn $\$ 1.45$ per roo lbs.; Bran $\$$ I.30 per Ioo lbs.; Feed corn meal $\$$ I.55 per Ioo lbs.; Cracked Corn $\$$ I.6o per Ioo lbs.

\section{CHICK FOOD.}

Question-What is a good chick food similar to the grain mixtures on the market for young chicks? 
Ansiver-Cracked wheat 25 parts, pinhead oatmeal I 5 parts, millet seed io parts, cracked corn Io parts, granulated charcoal 5 parts, chick size grit Io parts, buckwheat 5 parts, rape seed 5 parts, broken rice 5 parts, cracked peas 5 parts, and ground beef scraps or other meat meal 5 parts. Some of these are not easily obtainable in all sections and the mixture should be made with the ingredients at hand and considering cost.

CLASSIFICATION OF DRESSED POULTRY.

QuESTIONS-At what weights are squab broilers, broilers, fryers and soft roasters received in the Californian markets?

ANSIVER-Squab broilers three-quarter $1 \mathrm{~b}$.; broilers I $1 \mathrm{~b}$. to 2 lbs.; Fryers I and one-half lb. to 3 lbs.; soft roasters 3 and onehalf lbs. and up.

THE AVERAGE PRICE, OF LIVE POULTRY AND MARKE'T EGGS.

QUESTION-What is the average price to the producers in California markets for live poultry and market eggs?

ANSWER-In the Los Angeles market (the best in the State) for the year ending June 3 oth, I906, the average price paid for live poultry was: broilers, fryers and roasters I5 cents per lb.; hens 12 cents per $1 \mathrm{~b}$.; old roosters 8 cents per $1 \mathrm{~b}$. The average price paid for eggs during the same period was 3I cents per dozen.

\section{WHITE ON CHICKS OF BLACK VARIETTIES.}

QUESTION-Should chicks of a variety which should when fully feathered be black show white markings when first hatched?

ANSWER-Yes, all chicks from black varieties show white down when first hatched. Many breeders claim that the more white they show as chicks the more green sheen they will carry in their adult feathers.

WHAT IS A STRAIN.

QUESTION-When can a breeder claim to have a strain?

ANSWER-A strain is a family of any variety of fowls bred in line by descent, by one fancier or a successor, during a number of years, that has acquired individual characteristics which distinguish it more or less from other strains or specimens of the same variety.

\section{LARGEST TURKEYS.}

QUESTION-Which is the largest breed of turkeys?

ANSWER-The Bronze. The standard weight for cocks is 36 lbs. and for hens $20 \mathrm{lbs}$.

\section{SEX IN DUCKS.}

QUESTION-How can the sexes be distinguished in ducks?

ANSWER-The first noticable feature in young birds is the drake's longer bill, neck and body and a more upright carriage. 
When about four weeks old the duck can be distinguished by her quack. The drake has a finer voice, and at four months the curled feathers appear in the tail.

GOVERNMENT WHITEWASH.

QUestion-What is the recipe for the whitewash used by the U. S. government for lighthouses, etc?

ANSWER-Slake in boiling water one-half bushel of lime. Strain so as to remove all sediment. Add two pounds of sulphate of zinc, one pound of common salt and one-half pound whiting, thoroughly dissolved. Mix to proper consistency with skim milk, if possible, and if not use hot water. Stir in thoroughly half pint of liquid glue and apply the wash while hot. It may be colored if desired, by using yellow ochre, ultramarine blue, lamp black, etc. It will compare favorably both in appearance and durability with a good quality of paint.

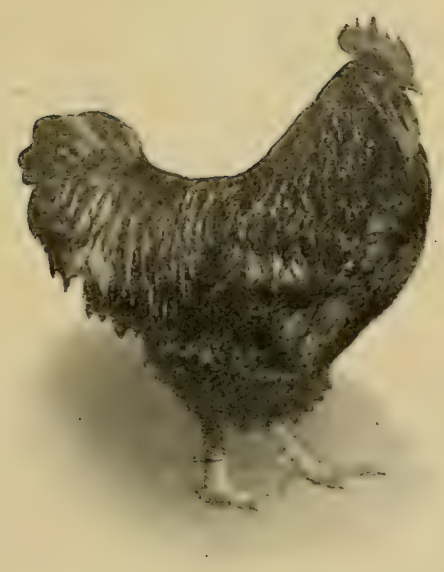




\section{The Egg.}

That the development of all animal life is done in the egg form most people know, but as to the various modes of development few are acquainted. In the mammal species the egg is retained throughout the period of incubation inside the body. In bird life the egg is enclosed in a shell, and incubation can be arrested for any period so long as the life is not destroyed. As the yolk becomes fully ripe the membrane which envelopes it becomes gradually thinner, especially around its greatest diameter, where

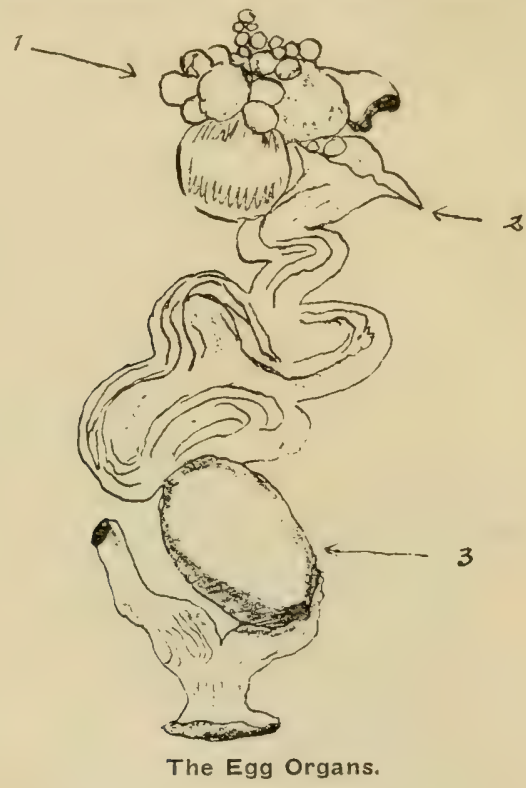

can be seen a pale circle or belt called the stigma. Finally the sac ruptures at this point, and the liberated yolk and germ incased in a very thin and delicate membrane, is received by the funnel-shaped opening of the egg passage or oviduct, (see figure 2), whose work it is to convey to the outer world, and on its way furnish it with every thing needful for its development and preservation. This organ in an ordinary hen is nearly two feet in length, but in the illustration is a little modified for convenience of representation.

It will be easily seen how two yolks may escape and enter the oviduct at nearly the same time, in which case they will 
most likely be developed in the same white and shell, thus causing a double yolked egg. The mucous membrane of the oviduct secretes a fluid which envelopes the yolk, and is added layer by layer as the egg passes on its way. This is called the white or albumen, and has many uses. It is greatly nutritious, and forms the chief nourishment for the chick during its growth in the shell, and as it is absorbed by the little creature it gives the fast growing body the necessary increase of room, seeing that it forms the greatest part of the egg when laid. It also guards the hatching egg against chill which would prove fatal when the hen leaves the nest, it being a bad conductor, and it protects the delicate yolk and vital germs from concussion and other injuries. At a further point of the oviduct the egg becomes invested with the skin or parchment like covering which is bound inside the shell, and consists really of two layers. This can be separated easily. At the large end of the egg they do separate, which thus forms the air chamber. This is small in a fresh egg but enlarges as it becomes older. In the last portion of the oviduct the egg becomes coated with that calcareous deposit which forms the shell. The now ripe egg passes into the cloaca (figure 3 ) and is ready to be expelled. If this portion of the oviduct be in an unhealthy condition, or the yolk is ripened by the ovary faster than the shell can be formed, then we get soft or unshelled eggs. As is well known, eggs vary in color according to the different breeds. This coloring matter is added over the solid ingredient. In somecases it is a deep, rich, pinky buff, in others brown spots or splashes, and some are covered with a kind of whitewash which, if rubbed with a rough cloth, will reveal a buff, brown tint underneath. All these things depend upon a peculiar condition of the secreting organs; so does the shape of the egg of each bird when finally laid.

\section{KEEPING AND MARKETING EGGS.}

The eggs, whether intended for market or for hatching, should be kept in clean cases, either the wire spring pattern or pasteboard fillers, and should be kept in a moderately cool room that is clean and well ventilated, and where the temperature does not go below 50 or above 60 degrees. This room should not contain an y vegetables, oil or other matter having strong odor, as such articles will taint the eggs. The eggs should be packed in the cases small end downward. Where they are intended for hatching, it will be a wise plan to turn them every day or two. For this purpose a revolving egg cabinet will prove a labor-saving essential. These cabinets are a practical invention, devised for the purpose of caring for eggs for hatching, providing means for turning the eggs with little expenditure of time and no loss 
through breakage or careless handling. These cabinets can be purchased in several sizes, from I 50 to 700 eggs.

In earlier times eggs, if sold at all, were marketed near the place where they were produced and many are still sold in local markets, but with improved methods of transportation the market has been extended and large quantities are now shipped to distant points. Special egg cases are required for carrying eggs long distances.

Eggs which are to be shipped, should be perfectly fresh and should never be packed in any material which has a disagrreeable or strong odor. All new laid eggs should be grated according to size and color. Musty straw, cardboard, bran, or other packing material having a disagreeable odor will injure the flavor and keeping qualities of the eggs packer in it. Keeping eggs near a lot of apples, or other goods having a pronounced odor, during transportation has been known to injure the flavor of the eggs and reduce their market value. Micro-organisms may enter the minute pores of the eggs and start fermentation which ruins the eggs for market purposes. Ergs which have been kept in a warm place, or where the germ has been started under a broody hen, will also become rotten, and the presence of a few such eggs in a case will result in a loss of price on the whole shipment. The normal mucilaginous coating of the surface of the egg protects it, and somewhat hinders the entrance of the organisms which start fermentation. This coating is removed or injured by washing the eggs and the kerping qualities of the eggs thereby diminished. For this reason it is not a good plan to wash eggs which are to be shipped some distance to market or which are not intended for immediate use.

\section{PACKING EGGS FOR SHIPMENT.}

For safe ways of packing eggs, our rule is to try to pack them so securely that if one lets them fall from the height of one's head to the ground none will be broken.

For the one, two and three settings and fifty, take a good, firm basket. They are called diamond market baskets, of eight, sixteen and twenty-four quarts sizes; and first line them with paper. Then put in a lining of excelsior, leaving just enough room to stan d the eggs in the center after wrapping each egg in a large sheet of paper. Take one page of a newspajer, putting the egg on one side in the center, giving the paper several turns, then fold over theone side of the paper, give it a half wrap sothe fold comes under, and fold the other side. This gives the egg many folds of the paper on each side, and the ends are firm, making a sort of box for the egg. To make them doubly safe, put excelsior between the eggs and on top, and then sew muslin over all, putting on a label. 
If you wish, you can buy a wooden box with large egg places and wrap each egg and use bran or light sawdust to fill. These are safe and less trouble to pack, but will cost more than twice as much in money. If you are a beginner and not are shipping extra fine eggs, you cannot afford the expensive boxes. We have had eggs broken in them also.

For the one hundred egg orders or over you can take fifties or you can use a light egg crate. If you do use a crate use only every other hole in the fillers, and be careful that when you begin a new filler you do not use the fillers directly over those you used in the last layer.

One of the points to have shipments go safely is to make the package firm. If they are too lnose the eggs will work together and they are sure to be broken. You will not hurt the eggs by pressing them a little, and at the sides of the fillers some excelsior should be used. If you prefer, you can use bushel baskets for hundred egg orders. but I have thought it would take more care to pack one of them safely than a crate. The one point would be as to which would be hancled the best by the express companies. It is a safe rule to use handle packages as much as possible, for they are by habit handled with some care.

Take the eggs as fresh as possible, and when they are waiting a few days for an order, be sure and put them where they will not dry out, for the inside of an egg should be full so it is firm as surely as the outside. I have found in years of shipping that eggs will hatch after long shipments-shipments of three thousand miles, if they are fresh and well packed. 


\section{Managemnt of Poultry During Summer Months.}

This subject naturally divides into topics: $I$, what fowls to keep; 2, how to handle them to best advantage. Both of these topics have to be considered with reference to future as well as immediate results.

Old Hens as Layers.-In connection with the question of keeping over for another year the hens now from a year to fifteen or sixteen months old, arises the old question of the relative value of pullets and hens as egg producers, a question which has provoked as much controversy and as much needless and pointless wrangling as any of the many questions each swiftly passing generation of beginners in poultry culture has to solve anew for itself.

There is no best breed of fowls. There is no best method of feeding or housing. We are many men of many minds, working under many different circumstances, with stocks of fowls that have experienced many different conditions. So we cannot all use the same rules, nor will any of us be wise to make hard and fast rules to govern in the conduct of our business.

Properly designated, a pullet is a hen less than a year old. The period for which results are usually compared is from the time the first pullets bagin to lay-generally October or November-until the next spring or early summer. Hence, in such comparisons, no account, as a rule, is taken of the performance of the hen, during summer and early fall, though it must be evident to every one that in considering the question we must consider what we may get out of the hens in the four months or so before the pullets are laying, as well as what we may get in the winter and spring.

Some authorities advise and some poultrymen make a practice of disposing of laying stock in the fall when the hens that were pullets in the preceding winter are sixteen to eighteen months old. We think it is much the better way to give the old stock a general overhauling before extreme warm weather comes on, and arrange at that time for the most profitable disposition of each of the several lots into which the flock will be divided. When the general clean-up is to be made in the fall the tendency is to leave in the flock many hens which should be disposed of without delay, the reason the poultryman gives himself for this being that, as the bulk of the lot is to be disposed of at that future time, it makes little difference if the few unprofitables are allowed to remain in it. Consequently some 
proportion of the fowls in each pen or flock are non-producers, adding nothing to the income, while consuming their share of the food, occupying room, taking time and attention of the poultryman, and, last but not least, adding to the risks of loss. for the idle, unproductive fowl is detrimental to the flock.

If as the poultry keeper reviews his experiences of the past year he recalls any of the following things as circumstances in the history of his flock of yearling hens, he is warranted in considering that an unusually large proportion of them are not good candidates to keep over: $I$, if they were not thrifty as young chicks; 2, if at any period of growth they were checked; 3 , if there was at any time during the year any serious sickness epidemic among them; 4 , if they were spasmodic layers; 5 , if after a period of good laying they suddenly fell off and were hard to get laying again; 6 , if they are now generally in poor condition.

In selecting from a flock of yearlings that have been generally in good condition, free from disease, and fair to good egg producers, take first those that are plainly in good health and condition, bright looking and presumed to be laying or about to lay. Hens that answer that description at this culling season we may say quite positively that it will pay to keep over.

Ventilation in the Poultry House.-Many poultry houses are so constructed that they cannot be thoroughly ventilated. Many that might be well ventilated by leaving all doors and windows open day and night are but partly opened in the daytime, and almost closed at night. Houses with the roosts ncxt low rear walls, and houses with deep narrow pens give very unsatisfactory conditions on hot sultry nights. In cool summers hens may do fairly well in such houses, but in hot seasons the lack of air in such quarters is very debilitating. A summer poultry house should be airy day and night; it must be so if the fowls are to do well and keep well in it.

Yards and Range.-Fowls keep in best condition in summer if they have good grassy range, with both sun and shade as they may want to take them.

They can, as a rule, be made more productive if confined to yards where the supply of green food does not exceed their actual needs, and fed well on a ration differing but little from that given during cool weather.

The most productive fowl, however, is not always the most profitable forwl. The item of labor must be considered, and the more yard room fowls are given the less close attention to their wants is necessary. No rule to govern the adjustment of labor to production can be given. It must vary under different circumstances.

If a man has nothing to do but take care of his fowls, or has ample time to give them all the care required under intensive methods, his best policy is to get the largest possible product from his hens. 
If in the use of intensive methods he gives time to poultry that otherwise would be devoted to other profitable work, he must decide just what division of his time will pay him best. Such decision requires some experience and some experiment in adjusting methods to circumstances. The essential thing in the early stages of one's work with poultry is to understand the need and advantage of striking the right bulance in the distribution of time to different kinds of work, and direct one's effort toward the gradual solution of the problem.

Consideration must also be given to the fact that, though by confinement and high feeding, hens may, as a rule, be made more productive than when given more liberty and lighter diet, the process wears them out faster, and it is not advisable to force in this way hens that are to be used the next season for breeding purposes, or indeed hens that are wanted to lay during the succeeding winter and spring. High feeding through summer should be carried to the limit only with hens that are destined to be sold as soon as they cease to lay profitably. With those we want to produce longer we must be more moderate, and must modify the diet, even at cost of reciuction in the egg yield, during hot weather.

The Effects of Change.-Another point to be considered is that a change of quarters or a change from one system to a quite different one, with perhaps changes in diet or method of feeding with the changing conditions, will at the same time better the condition and the egg yield of fowls.

Again and again poultrymen have taken breeding hens after months of heavy laying in confinement, put thein on range to recuperate, not caring whether the hens laid or not, and found that after a little rest they began to lay, and laid well for a long time, when if left in their old quarters they would probably have done nothing. Good laying under such conditions does not seem to take as much out of the forwls as the same production in confinement. Such a change is not a sure way of making hens lay in summer, but it secms to do so often enough to make it worth a trial when other methods fail.

Green Food.--Ordinarily fowls may be allowed all the green food they will eat, both winter and summer. Sometimes, however, when the weather is extremely hot they will, if liberally supplied with green food, fill up on it and take too little grain to sustain egg production at their usual mark. If egg production is to be maintained, it is best at such times to feed green food only after the fowls have been fed on grain.

Again, fowls on a range well supplied with grass and insects are apt to get in the habit of foraging early, maintaining themselves on insects and grass, and lay very little. The best way to do in such cases is to keep the hens up until they have had one good feed of grain. If practicable the end sought may be gained by increasing the number of fowls on the range, thus 
making the supply of food each secures by foraging enough less than a comfortable sufficiency to keep it ready to take a fair ration of grain once or twice a day.

Animal Food.-A range must be very good indeed to furnish all the animal food they need to fowls that can get all the green food they need on it. Hence under ordinary conditions it is more necessary to supplement the animal food of hens on range than to provide special supplies of green food, and we think hens on range in summer will stand even heavier feeding of concentrated, prepared meat foods than when confined in winter. For hens that are closely confined it is safer to reduce the proportions of prepared animal food, and if it is desired to feed meat heavily feed at least a part of fresh meat or green cut bone, which contain large percentages of water. In feeding concentrated animal foods at this season special attention should be given to the quality of the articles used. Anything of this kind that is not sound, sweet and good will produce bad effects more quickly in hot weather than at any other time.

Shade.-It is absolutely necessary that birds be furnished with abundant shade during the summer months. Trees and natural shade of course is better than "made shade," but where yards are located where there are no trees or other shrubs shade should be provided. One of the best "made shades" we have seen was made from palm leaves placed on a light frame. This should be placed high enough from the ground to allow the air to circulate under the frame and at the same time be low ennumh to kee, the sun from the hirds when they are under the shade. The birds enjoy a "dust bath" made by wetting up a portion of the grouid under the shade and when partly dricd spade it up; this is of course an addition to the regular dust bath made of dry road dust, and does not take the place of the dry bath. 


\section{Breeds Best Adapted for California.}

The selection of a breed best suited to one's purposes, while an important step in establishing a poultry plant, is much a matter of taste, for the breeder must have a particular liking and unbounded faith in the particular variety or varieties he is handling, just as a salesman must have faith in his particular line of goods before he can successfully induce others to see them to the best advantage. This applies to the purely commercial side of the business as well as to the breeder who expects to establish a plant solely for the raising of breeding stock and eggs for hatching. At the same time one must consider the conditions under which he is to breed his birds, the location of the plant as applied to climatic conditions, the particular branches of the business which are to be ratered to, etr. One must decide, before making the selection, whether he is to build up an egg farm, an egg and meat business. raise bioilers, roasters, ducks, turkeys or estatlish a plant for the furnishing of breeding stock and eggs for hatching (or a selected combination of the different bianches). California has such a wide variety of climates that it is not possible to recommend any particular varicty or varieties that are adapted to all sections of the state. This makes it possible to produce almost all of the Standard varieties in our state; where we have the cliniate of New England in our high altitudes to the dryest of deserts and the tropical climates in the valleys of our sea boards.

While we do not contend that this or that particular breed can not be raised with profit under any climate we know from experience that some breeds do better under the climatic conditions of certain sections than others when given the same care and attention. Therefore we believe it wise for a breeder to select a variety best suited to his particular climatic conditions.

Be careful in selecting your breed or breeds and when the selection is made stick to that variety and make the most out of it. Start with the best stock obtainable; cheap stock is high at any price whether they are intended for market or for breeding show stock.

\section{BREEDS BEST SUITED FOR THE EGG FARM.}

For the exclusive egg farm breeds in the Mediterranean class are acknowledged to be preeminent; the breeds comprising this class are the Single and Rose Comb Brown, White and Single Comb Brown, White, Buff, Black and Silver Duckwing Leg. 
horns; Single Comb Black and White Minorcas and Rose Comb Black Minorcas; Blue Andalusians, White Faced Black.Spanish and Mottled Anconas. All of the varieties in this class are nonsitters and have tested egg rec'vrds of from 200 to 280 eggs per year per hen.

\section{WHITE LEGHORNS.}

Single Comb White Leghorns have become very popular as an all round egg farm hird for California and are surely worthy of the praise that has been given them. The White Leghorn has been bred along lines to produce a large framed high stationed bird, which has given them something of a lead over the other varieties in this class both for the production of large eggs, beauty and all around usefulness. A flock of these birds, with their snow white plumage and sprightly appearance, furaging

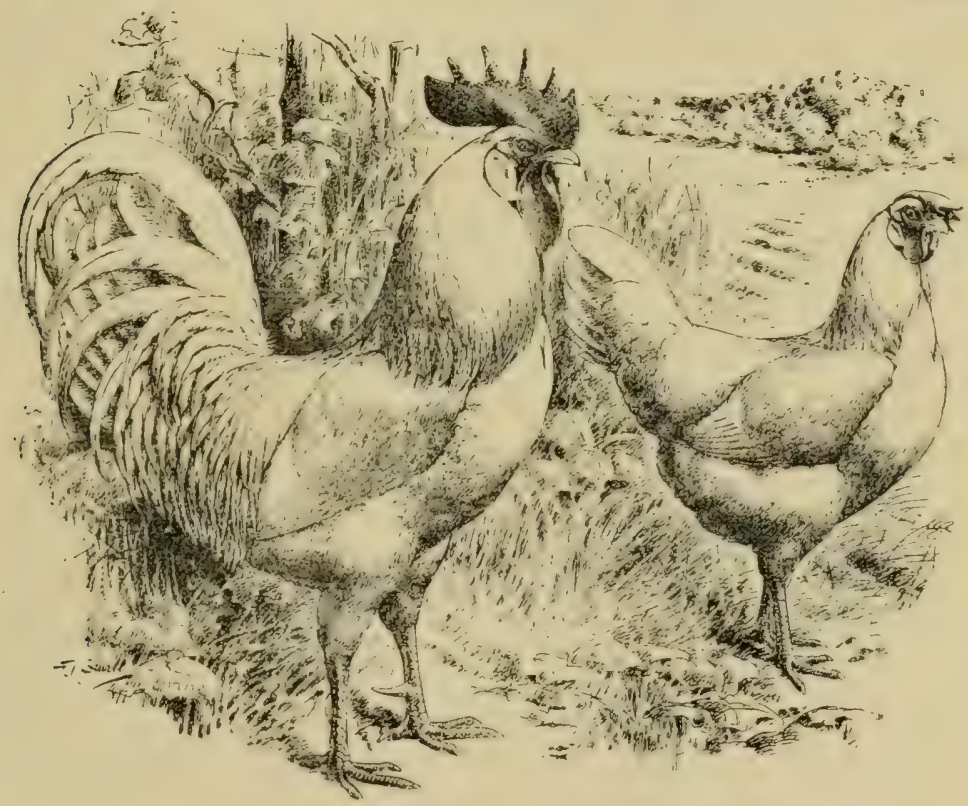

Single Comb White Leghorns.

on a California alfalfa field makes the ideal picture of beauty and productiveness. While White Leghorns are considered easier to breed true to feather than some other of the varieties carrying several color pigments in their plumage, high class specimens in this variety are highly prized and really are as rare as some of the breeds cunsidered very hard to breed. The advantage in breeding the White over some of the Parti-colored breeds is that the percentage of culls is very much smaller and the average quality of the flock easier to maintain. 
A Standard White Leghorn should be a bird standing well up on legs, sprightly and showing great alertness and graceful curves. They should be essentially a bird of trim build and specimens running to the over large and coarse appearance depart from the true type and are also apt to be less valuable as egg producers. We do not mean by this that White Leghorns should be of diminutive size in order to insure their productiveness; on the contrary we favor a bird of high station, long back, well filled out frame, but such a bird can and is being bred with splendid Leghorn type, showing none of the coarseness which destroys the bird both for the show pen and exclusive egg production. The plumage should be snow white in all sections; the ever troublesome " "brassiness," while not as prevalent in the White Leghorns as in some of the other white breeds, is nevertheless found in many fine specimens, especially in the males, and should be carefully avoided in mating the breeding pens. Anuther common defect and one that should be considered carefully before a bird (and this applies to all the Mediterraneans) is placed in the breeding pen is the carrying of the tail so that a portion of it projects towards the neck, beyond the perpendicular line at the juncture of the tail and back; this is called "Squirrel Tail" and generally goes with a short back which makes it a defect to be avoided in both show and utility birds. The Standard calls for the tails of Leghorn males to be carried at an angle of forty-five degrees from the horizontal and in the females at an angle of forty degrees. The back should be of good length, the saddle raising in a short concave sweep to the tail. The breast should be very full and have the appearance of being carried well forward. Ear-lobes should be of medium size, as smooth and fine in texture as possible and in color white or creamy white, we prefer the clear white lobes as we have found that the birds showing the creamy lobes are the ones must apt to show "brassiness" in plumage. The cumb of the male should be of medium size rather fine in texture with five even points: standing upright and firm on the head; in the females the comb is the same with the exception that it should fall gracefully to one side of the head. A very striking thing about a well bred White Leghorn is its beautiful bright yellow beak and shanks and toes; in contrast with the snow white plumage these show up to the best advantage. In some sections the soil and climatic conditions are somewhat against the yellow Ieg and beak birds and cause them to fade into a light color more white than yellow. We know of a number of flocks however that have been bred to a point where neither soil or climatic conditions had any affect on the color of legs or beaks and any breeder can get fine color in these sections by careful selection of his breeding birds. The mixing of the yellow pigments for these sections and at the same time retaining the clear white plumage has been a problem that the breeders of many 
of the white varieties have had to cope with, and the breeders of White Leghorns have solved the questiun perhaps better than any of the others as an inspection of the large flocks throughout the country will prove. It is these small defects in many cases unimpurtant in themselves but when a number are found on the same bird, as they frequently are, they make the production of a really first class White Leghorn and interesting study to both the fancier and the commercial breeder. It is in the size of their eggs that the White Leghorns surpass all the other varieities in the Leghorn family. Some strains have been bred to a high quality in this regard. We know of several breeders who have flocks that produce eggs that weigh six and seven to the pound. Such eggs with their clear white shells always command a special price in the California markets. White Leghorns are generally handled on an egg farms in colonies with free range, and are at their best when called upon to forage for a part of their living; at the same time many breeders have found that they got better results from birds kept in confinement. None of the Leghorns are easily put out of condition by improper feeding, especially by overfeeding, and this is undoubtedly the reason many breeders are able to get a greater egg yield from them than from some of the heavier varieties. This is particularly true with birds in limited quarters. Many of the largest and most successful egg farms in California breed White Leghorns exclusively; particularly is this true of the large plants in and around the great poultry district at Petaluma and throughout Central and Southern California.

\section{BROWN LEGHORNS.}

As we stated in the opening part of this chapter the matter of taste should go a long ways in deciding the variety to be taken up. In this regard the Brown Leghorn is a good example fur they have many attractive features not possessed by the White Leghorns and at the same time have all good qualities found in their solid colored cousins. They have one very important advantage over the Whites, a color that withstands the hottest summers without fading or becoming dirty with the dusty atmosphere. For this reason they are popular with breeders living in California districts where the summers are warm and of lasting duration. Not that the Browns do not do well in the cooler and damper sections, for they have been one of the most popular California breeds for years and there is probably no better place in the world to produce high class show specimens of this variety than in this state, a fact which has been emphasized the past few years by California Brown Leghorns carrying off some of the bast prizes in the largest eastern poultry exhibitions. That this variety has great producing and beauty qualities no one who has bred them will contradict; that they 
are especially adapted to the climate of California is an acknowledged fact. A well known eastern poultry expert while visiting California was asked what impressed him the most in the poultry line and he promptly replied, "its great possibilities for producing world beating Brown Leghorns." Brown Leghorns are not an easy variety to breed to standard requirements, in fact the females are probably as hard a proposition in this regard as we have in standard poultry culture; however, this only makes them more attractive to the fancier as it is a great honor to produce good specimens and they always command good prices. Unlike some of the other fancy-and-hard-to-produce varieties the Brown Leghorns are great producers and are

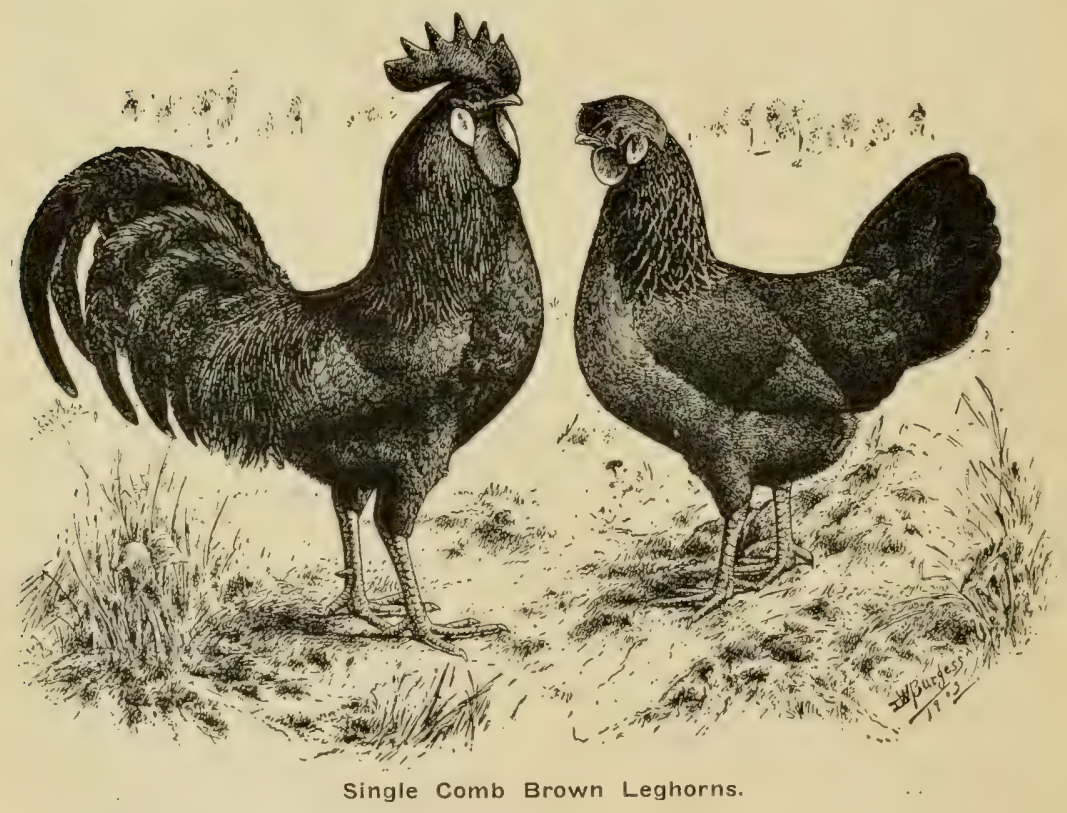

noted for their egg laying qualities; therefore a breeder can have a profitable flock of birds and at the same time satisfy his hobby from a strictly fancy point of view.

The past few years have seen great strides in the qualities of the Brown Leghorns, notably in the increased size and in the size of the eggs they produce. These points were somewhat overlooked by the breeders of Browns for a number of years, in their effort to improve their beautiful color points; as the breeders of the White Leghorns had been making a special effort for size of both birds and eggs, this variety gained somewhat of a lead over their beautiful cousins in the yards of the commercial breeder. At the present time the two varieties are held in about equal esteem by both the fancier and the com- 
mercial breeder. A choice is usually made from a fancy for one or the other color than from any difference in their producing qualities. In shape and general type the Brown Leghorn is identical with the Whites. The color of the male is very attractive and makes one of the most beautifully plumaged bird in the standard varieties. The hackle should be of a deep rich brilliant red with a distinct black stripe down the middle of each feather, the black not to extend over the end of the feather but come to a point near the end; the red to form a lacing around the entire outer edge of the feather. The back should be rich red, the saddle same color and striped as described in the hackle. The breast should be a rich black without any splashes of foreign color. Wing bows should be same color as the back, the flight feather, upper part black and lower edge a reddish brown, when folded this should form a well defined black wing bar. The tail should be a greenish black; undercolor slate; legs, toes, comb and earlobes same as in the White variety. The female Brown Leghorn is the pride of the fanciers heart and while not as attractive to the eye as some of the other birds a well bred flock of Brown Leghorn pullets or hens are a sight that will please any lover of web and feather. The color scheme of the Brown Leghorn female is a bird clothed in a soft brown plumage in back and wings (lower web of flight feather on wing black but not showing when wing is folded) the brown finely penciled with dark brown, the lighter brown predominating, producing an even soft brown tint. The breast should be a rich salmon; hackle a rich orange yellow, striped with black the same as in the male; the tail should be black except the two highest main tail feathers which are penciled as on back; The main defect in most specimens is the presence of shafting showing in the center of the feathers in all sections. Birds selected for breeding purposes should be as free from this defect as possible. The dark brown penciling should not have a coarse appearance as it is important to produce an even soft brown tint and to do this there must be a fine intermixing of the light and darker brown. In legs, toes, comb and all other sections the Brown Leghorn females are the same as the White Leghorns.

Brown Leghorns (as are all of the Leghorns) are rapid growers and the pullets are at a laying stage before many of the larger breeds are fully feathered. The up-to-date Brown Leghorns lay a large white shelled egg; and lots of them; some breeders contend that they are better winter layers than any of the other varieties of Leghorns but we are of the opinion that there is more in the strain, and the way it has been bred, than in the difference in the varieties as a class. In fitting a plant for the producing of eggs for market no mistake will be made if Brown Leghorns are selected. 


\section{BUFF LEGHORNS.}

Here is a most excellent bird that has not received the attention that it deserves from breeders in California. Buff Leghorns are well adapted to this climate and those who have bred them find that they are one of the greatest egg producers we have. Of all the Buff brecds with the exception of the old Buff Cochin, the Buff Leghorns breed the truest to correct color. They are of good size, not quite as large in frame as the Whites or Browns, and are one of the best in the Leghorn family to stand close confinement; at the same time they are excellent

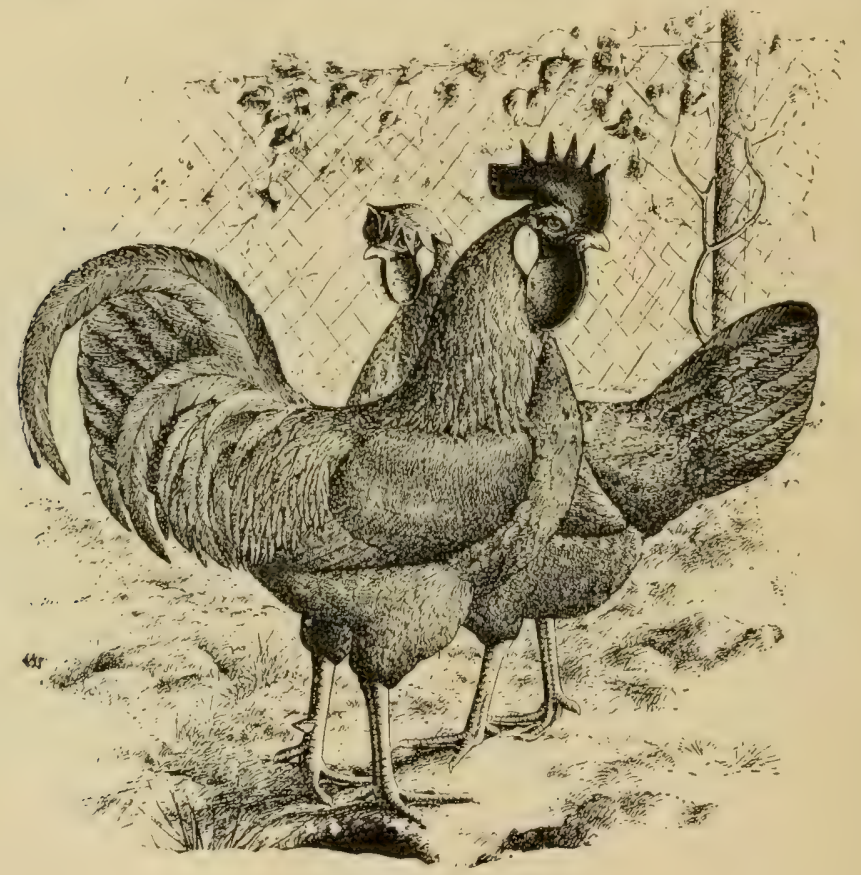

Pair of Buff Leghorns.

foragers; their eggs are usually of good size and they commence to lay at an age that makes the pullets soon produce their value in eggs. In general type they are like the other Leghorns; plumage should be of a rich golden buff in all sections producing a harmonious blending of buff color throughout the entire plumage. Birds with mealy plumage and those with shaft of feather showing are to be avoided; an even shade of buff in all sections is to be most desired. There is no variety among the standard breeds that is hardier, grows faster or lays more eggs in a year than the sprightly Buff Leghorn and there is no breed that will better suit the purpose of the man who wants to start an egg 
farm or the fancier who wants a flock for beauty that are producers as well. Again it is only a matter of taste.

\section{BLACK LEGHORNS.}

The history of this very beautiful and profitable variety proves conclusively that the work of the fancier very materially affects the progress of a variety not only as a strictly fancy bird but as a commercial fowl as well. In the case of the Black Leghorns we have a very attractive bird, with all the good qualities of the other Leghorns, yet it is seldom found in any numbers and we do not know of a single case where they have been used on an egg ranch.

While we are willing to admit that the popularity of the Black Ninorcas has in a measure retarded the growth of this variety, we do not believe that it is responsible for keeping them in the rear of the money-making variety procession as they are found today. To those who have not followed the history of the standard varieties of poultry it may seem very strange that a small matter of color of shanks should retard and practically ruin the usefulness of a variety possessing wonderful producing qualities and a member of one of the most popular classes in the American Standard.

This, however, is the case: For years past the American Standard makers have persisted in demanding that Black Leghorns with shanks other than vellow or yellowish-black should be disqualified in the show room. As nature never intended that birds with black plumage should carry yellow pigment it has been next to impossible to produce specimens that would do for exhibition purposes and in consequence they have been neglected by the fancier and have up to this time received no attention from the commercial breeder. We do not want to be understood as saring that the Black Leghorns have no admirers, they are found in the yards of many prominent breeders and their good qualities are praised by every one who has ever given them a trial. There is a movement on foot to change the present Standard and when that is done Black Leghorns should take their place with the other popular varieties in the Leghorn family.

\section{SILVER DUCKWING LEGHORNS.}

This is the latest member of the Leghorn family to be admitted to the Standard but they have already made rapid strides towards popularity and have many admirers in California where they have been given a thorough test and found equal to their older cousins. The Silver Duckwings are considered the most attractive variety in the Leghorn class and they certainly make a showy appearance in their combination of glossy black and silvery white plumage. 
In all but color the Silver Duckwing Leghorn should be identical with the other varieties in the Leghorn class. The color of plumage in males should be: neck with the exception of the hackle should be rich, glossy black; the hackle is silvery white with a black stripe down the middle of each lower hackle feather. Back is silvery white; cape black; saddle silvery white; breast black; body and fluff black. The wings should be glossy black and silvery white with a distinct glossy black bar across the wing. Main tail lustrus black, lower coverts silvery white. The females are somewhat different in color markings from the males. The neck with the exception of the hackle should be a light salmon color; hackle silvery gray with a black stripe extending down the middle of each feather, the back should be light gray showing no dark marks or bars. The breast is light salmon shading to gray towards the outer edge. wings should be light gray, free from dark bars, marks or red. Tail should be black with the exception of the two largest tail feathers which are light gray.

\section{BLACK MINORCAS.}

This is the largest bird in the Mediterranean class. The Standard demands that they weigh, cocks $9 \mathrm{lbs}$; cockerels $71 / 2$ lbs., hens $71 / 2 \mathrm{lbs}$; p pullets $6 \mathrm{r} / 2 \mathrm{lbs}$. This variety is not used on egg farms as much as their good qualities justify. They are equally as prolific egg producers as the Leghorns and lay the largest egg of any of the Standard varieties. A case or basket of these clear white, extra large eggs always attracts the attention of the buyers and easily command better prices than the average run of eggs. The Black Minorca of today is undoubtedly the Black Spanish of fifty years ago. The fancier built up a line of the old bloud on the lines of our modern White Faced Black Spanish and another line went on as it had always been bred for commercial purposes. This latter line resulted in our modern Black Minorcas.

In addition to the number and size of Black Minorca eggs they are to be credited with beginning to lay at a very early age, and continue to be profitable layers for a longer period of time than almost any other bird of which we have knowledge. They are excellent foragers, strong and active, always on the alert for any natural advantages that come within their reach, and having little tendency to lay on fat, the food they consume gives profitable returns in the form of eggs.

The white skin, somewhat marred by the black pin feathers and black legs, is a handicap from the market poultry standpoint, but the flesh is of an excellent flavor, deliciously tender in the younger birds, and with such a considerably larger amount of flesh than is found in the Leghorn it is, as we stated, a matter of a surprise that the Black Minorca is not more generally utilized as an egg-farm breed. 
Minorcas have a distinct and marked type of their own and while they are built on the same general lines as the other varieties in the Mediterranean class are really very unlike them in shape and symmetry. Birds of the short legged. closely built type should be avoided for the real Minorca type is a bird with a long body set upon a pair of firm, muscular legs making a bird

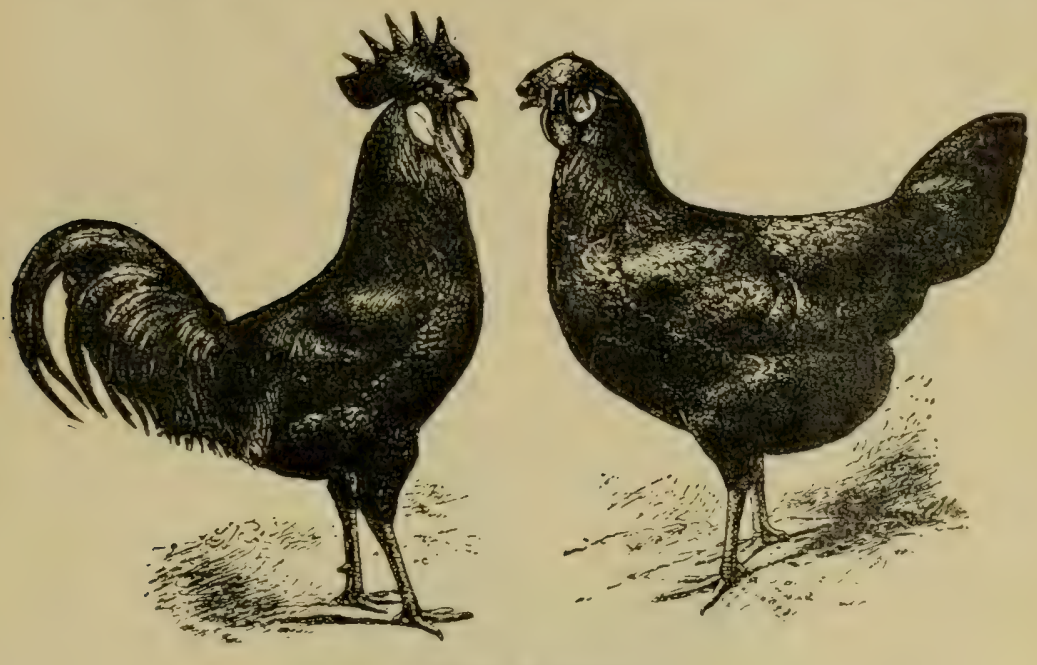

Black Minorcas.

with a powerful looking body. The plumage is beetle black in all sections, showing as little trace of purple barring as possible; they are a closely feathered bird and their feathers should be smooth on the surface. Comb should be large and have six distinct points. Wattles should be in proportion to size of comb; ear lobes white; dark colored legs and pinkish-white skin.

\section{WHITE MINORCAS.}

The Whites are identical to the Blacks in all but weight which is one pound less in cocks and hens and cockerels and pullets. They are not bred so extensively as the Blacks but have all their good qualities and to those who wish a white bird they prove great money makers. They make an ideal bird for breeders who are compelled to keep their birds on alkali soil as their legs are of a color that can not be faded by soils or weather.

\section{BLUE ANDALUSIANS.}

California is known the world over as the American home of the Mediterranean class; her Leghorns, Minorcas and Spanish have been praised by the best poultry judges the world affords, but for some unaccountable reason California Andalusians have had little notice. This is undoubtedly because no 
California Andalusians have been shown at the big Eastern shows, and we predict that when some enterprising California Andalusian breeder takes a string to New York, Boston or Chicago there will be a stir among the Eastern Andalusians breeders.

California breeders of Andalusians have made the most of their favorable climatic conditions, and we feel sure that no other State in the Union can show so many really top-notch Blue Andalusians as California.

A well-marked typical Blue Andalusian is hard to beat for either beauty or utility, and we have never met a breeder who gave them a place in his yards that was not enthusiastic over both their beauty and money-making qualities. The Andalusians should have been produced on American soil, as it is the only variety in the standard carrying the national colorsred, white and blue-red in face, comb, wattles and eyes, white ear lobes and blue plumage.

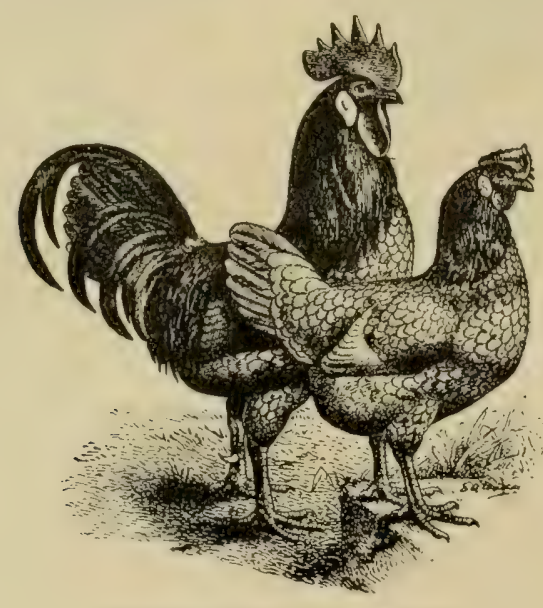

Standard Blue Andalusians.

Their origin dates back to the eighteenth century, when they were first introduced into Great Britain from Andulusia, a province in Southern Spain. It is a disputed question as to their blood make-up, some contending that they were the result of a mixture of White and Black Minorcas, while others claim that they were bred long before either of these two breeds, and that the Minorca is an offspring from the Andalusian. We are inclined to think that this latter is the more correct of the two theories, but we know that during latter years the Minorca cross has been used, in some strains, to increase the size. This was many years ago, and the birds as bred today show none of the earlier crosses, and possess a type distinctly their own. 


\section{ANCONAS.}

While this variety has only recently been admitted to the American Standard, and is as yet only found in limited numbers in this country; they have been popular in England for years and it is only a matter of time when they are sure to become one of our most popular egg farm breeds.

"They are a handsome black and white mottled fowl, approaching the Leghorn in shape, with golden yellow shanks and beaks, large, bright-red single combs, and white ear lobes. They have a wild, peculiar expression, and are as beautiful and interesting as useful. As early as $185_{2}$, as appears from a table in the English Agricultural Gazette, four birds of this breed produced in twelve months 928 eggs, or 232 for each hen, a record which if correct, puts in the shade on any of our present breeds or strains. They have always had the reputation of being wonderful layers of medium sized eggs weighing more than those of nearly any breed irrespective of size. In the -Government Experiment Station in N. S. Wales they have surpassed all other breeds as egg producers. They are short and stout, extremely hardy, of rare beauty and peculiar attractiveness, and are much admired by those "who know," both for utility and exhibition purposes.

The young exhibitor should be careful how he mates his birds if he wants to breed something worth showing. The cock should be darker than the Standard requirements, and the hens a good medium shade of under color and quills and fluff down to the skin should be black and not white, which is so often seen in inferior specimens. The black should be glossed with beetle green and the spots on the shanks distinct. The comb must be of medium size with 5 serrations, and it is to be hoped Ancona breeders will not adopt and judges will not encourage or permit, the huge beefy comb of the Minorca type which is quite out of keeping with the general character of the Ancona. The face, except the ear lobe itself, must be a sound bright red free from any suspicion of white. Anconas carry their tails rather higher than most birds, but a tail that is held over the back, squirrel fashion, is positively objectionable and should be avoided. The white mottling should be as clear and distinct as possible and free from any slatey tone.

The eggs of mature Anconas of the original imported stamp are as large as those of the Minorcas, averaging two ounces in weight. Frequently seven eggs weigh a pound.

The Ancona chick grows with amazing rapidity, and is very precocious and independent when reared by artificial means. I think that they learn to eat sooner than those of other breeds. They require plenty of run, and at the age of seven or eight weeks the sexes should be divided.

Whether considered as a utility or an exhibition bird the Ancona with proper attention is one of the most popular and profitable breeds. 


\section{HAMBURGS.}

These are in what is termed the Dutch Class which comprises Golden and Silver Spangled, Golden and Silver Penciled, White and Black Hamburgs.

The Silver Spangled variety is the most popular in this country and, while they are a small framed, short legged bird. they lay a good sized egg and their egg laying records have never been surpassed by any other standard variety.

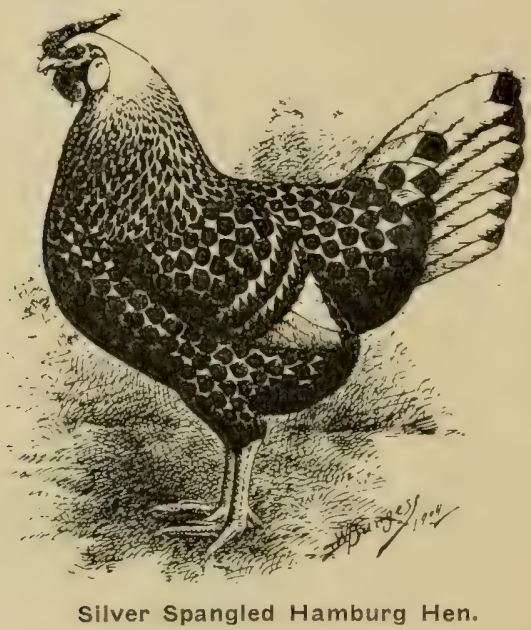

As a beauty breed they are without a peer, with exceedingly graceful type and a color very exact in its design (this applies to all the varieties in the class). The general markings on both male and female is a clear, silvery white, free from mossing or lacing, each feather ending with a large, black spangle which should be in proportion to the size of the feather.

All of the varieties in the Hamburg family make excellent egg farm birds and those who admire these beautiful little birds will make no mistake in taking them for their money-makers.

\section{THE GENERAL PURPOSE BREEDS.}

Under this head come the varieties that are neither very large nor very small in frame; that are not non-sitters but lay eggs enough each year to make them profitable as egg producers. The advantages in breeding the all-purpose breeds is that they can be turned to profit in a number of different ways. The pullets make profitable egg producers being specially valuable as winter layers when eggs command the best prices; at the end of their usefulness as egg producers they can always be turned into money at good prices, for table purposes (hens of this class 
average $I 2$ cents per $1 \mathrm{~b}$. in the California markets). The cockerels and culls mature early and are always saleable at fancy prices for meat. Then there is the demand for well marked birds for breeding purposes, which is increasing each season and promises to tax the production of California standard poultry plants for years to come. The varieties that comprise the general purpose classes are the Barred, White, and Buff Plymouth Rocks; Silver, Golden, White, Buff, Partridge; Silver Penciled and Columbian Wyandottes; Rhode Island Reds and Buff, Black and White Orpingtons.

\section{PLYMOUTH ROCKS.}

The weight of all Plymouth Rocks are as follows: Cocks $91 / 2$ pounds, cockerels 8 pounds, hens $71 / 2$ pounds, pullets $61 / 2$ pounds. Head is of medium size with a fairly broad crown, beak is stout and well curved, eyes are medium and clear rich bay in color, comb should be set firmly on the head, straight and upright, and should be in proportion to the size of the specimen, neck of medium length, arched, tapering, with abundant hackle to the shoulders, back is broad, of fair length with a concave sweep

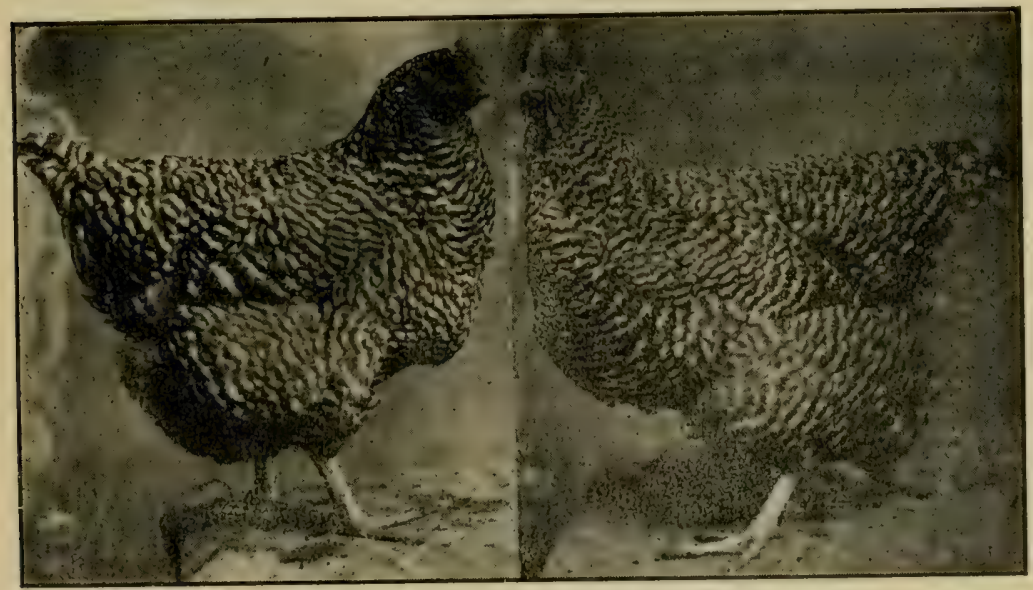

Pair of California Bred Barred Rocks.

to the tail, breast is deep and well rounded, body of medium length, full and compact, wings of medium size, well folded, tail not too long and fairly well spread, carried in such a manner as to be on an apparent angle with the back, legs and toes of medium length and straight, and bright in color.

The Plymouth Rocks are pre-eminently an all-purpose breed. They are not only great layers of good sized brown eggs, but they take high rank as meat producers also. For egg-farm purposes it would be difficult to name a breed combining great 
laying ability with meat producing qualities in so high a degree, and they are a most satisfying bird in every sense of the word. The Plymouth Rock is a "made" breed, and originated in a cross of an American Dominique cock on black Java hens. The "Cuckoo" marking of the original Plymouth Rock was received from the Dominique male, and the size, station, single comb,

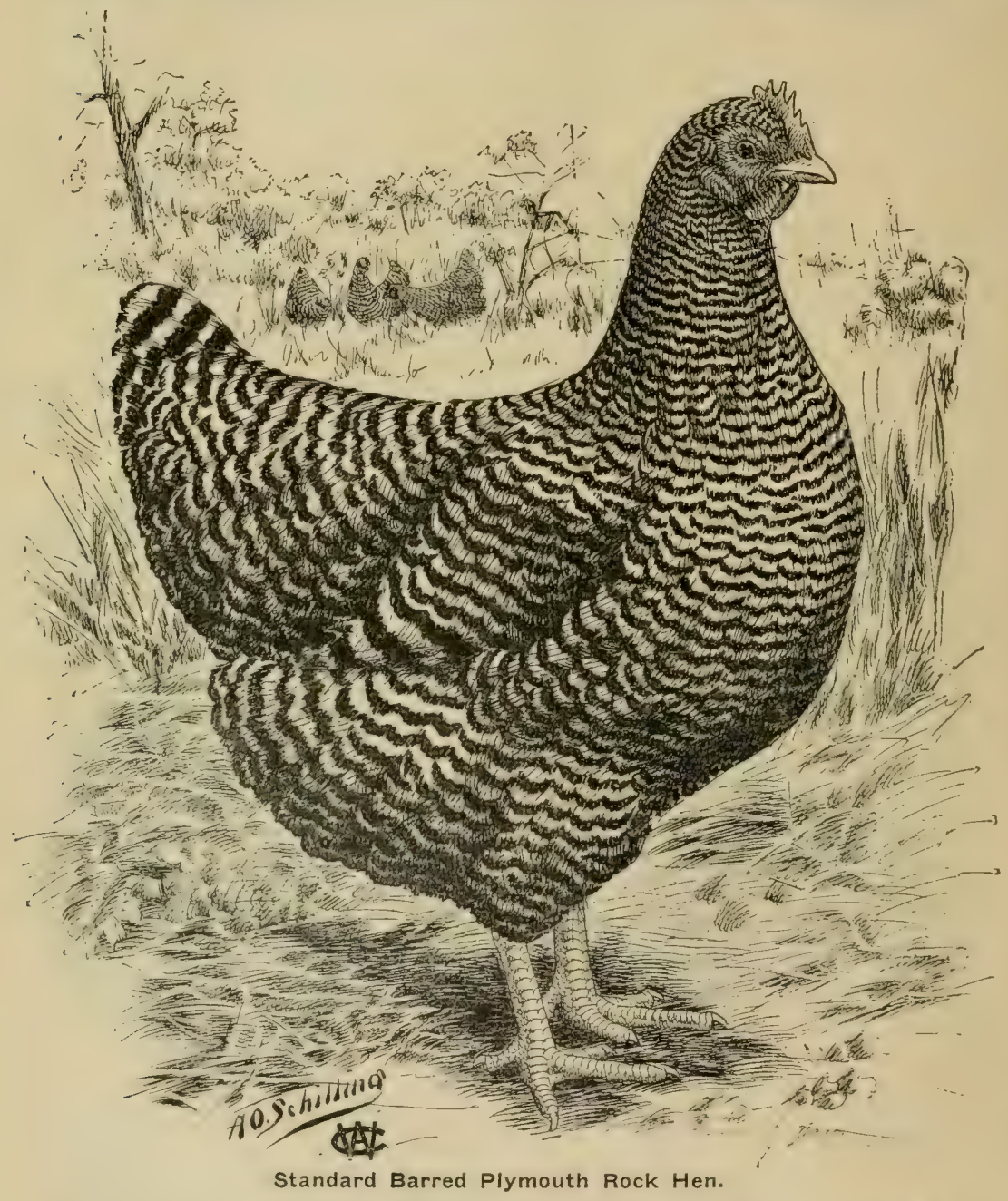

etc., from the dam. It is practically certain that the blood" of other breeds has now and then been introduced into the Plymouth Rocks, which has given them size, type, greater uniformity of plumage and the desirable yellow beak and legs. Their remarkable hardiness is one of their strongest claims to 
popular favor; being an American breed, accustomed for many generations to the extremely changeable and trying New England climate, and being thoroughly acclimated, they would be placed among the very first for hardiness and vitality. Writing of them nearly twenty years ago, the Americun Poniltry Yurd said: "The perennial popularity of the Plymouth Rock is something wonderful to those who do not know its real merits, but to those who do, to those who know that it is hardy, healthy, vigorous, prolific, excellent for the table and thoroughly adapted to the requirements of an American market and an American climate, there is nothing wonderful at all."

\section{THE BARRED PLYMOUTH ROCKS.}

5.: The Barred Plymouth Rocks, the original of the several varieties of Rocks, are the most popular and most widely bred variety of fowls in the world today. They enjoy the distinction of being the first breed of domestic fowls produced in America and their eminently practical qualities have won for them and their sterling merits have held a place in the very front rank of popular favor; they are noted for being bred by a greater number of persons and in greater numbers than any other one variety of fuwls.

"The Barred Plymouth Rocks commend themselves to the lovers of useful breeds. Of all our domestic fowls, this breed stands the highest for general purposes. They almost vie with the Asiatics in size, the Leghorns in egg production, the Dorkings in quality of flesh and the Dominiques in hardiness and adaptation to climatic changes. They combine more useful qualities than any other breed known to us, and fill the void between the size and weight of the Asiatics and the European fowls."

The Plymouth Rock is a product of American skill and breeding, and there is no other variety we can put on the markets of the world with so much pride, and none other is received at our shows by foreign fanciers with so much favor. They have taken their place at the front without need of booming, and today stand acknowledged without many equals, as one of the best general-purpose fowl. They are rapid growers and make plump, juicy broilers at from eight to twelve weeks old. They have no successful rival among the pure breeds as a market fowl. They are great favorites with farmers and market men, who breed this variety more extensively than all other pure breeds combined. The popularity of the Plymouth Rock as a fanciers' fowl has never been reached by any other breed. Its popularity is based on its practical utility worth, and as a practical fancy fowl, the Barred Plymouth Rock has come to stay. They are excellent all the year round layers, and will lay as many eggs as any breed that incubates and rears its young. In some 
of the smaller breeds we may get better layers but less size. The larger breeds give us no more eggs, if as many, and are later maturing and lack the sprightliness and elasticity of movement so much admired in the Plymouth Rock. The Barred Plymouth Rock class at our American shows is nearly always the largest, and the birds usually command a higher price than other American breed, which proves their sterling merit.

New breeds have come and gone, but the Barred Plymouth Rock with its good qualities remains invincible. They are

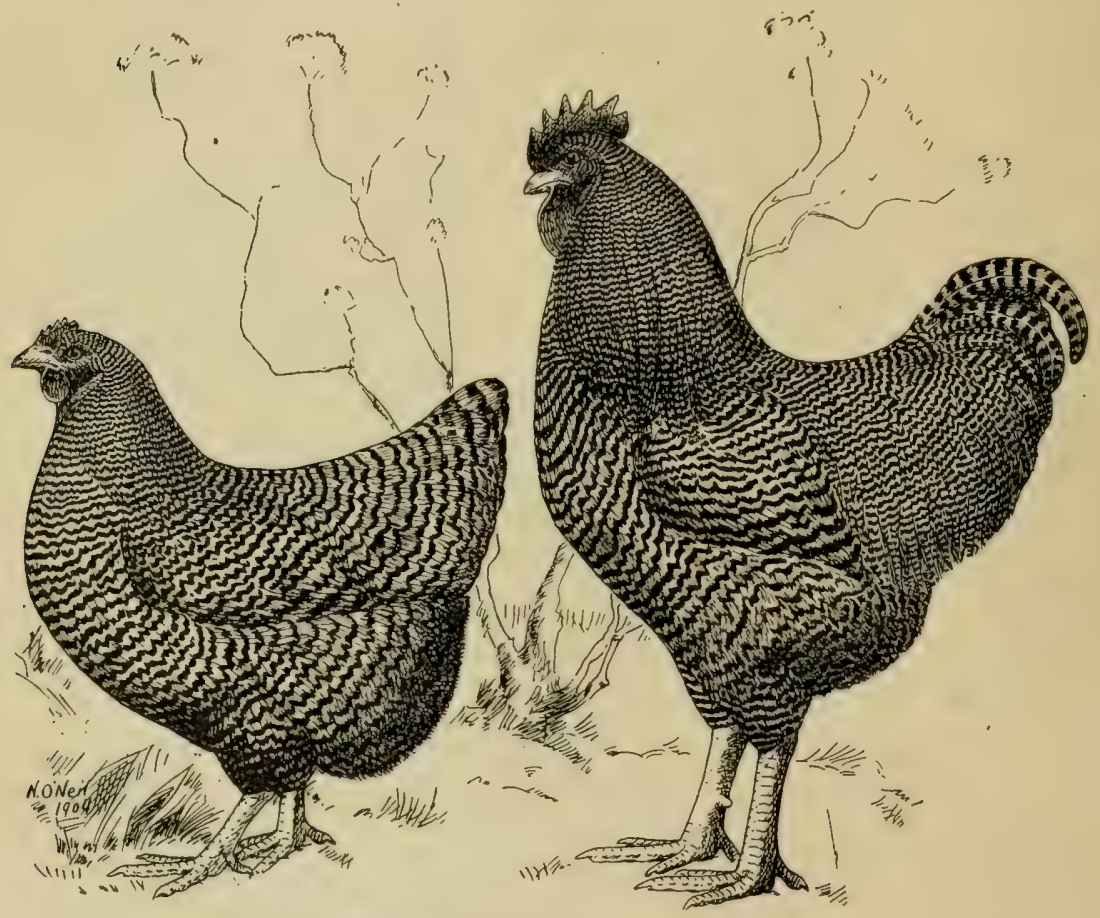

Standard Barred Plymouth Rocks.

practical fowls, well suited to the wants and conditions of those who desire eggs, meat and feathers combined in one breed.

The general verdict of Plymouth Rock breeders can be encompassed in the words of a noted fancier and judge. "The Plymouth Rock is, beyond all question, the best general-purpose fowl of all the breeds before the public. They have been before the public many years, have borne the competition of other fowls, have been subjected to every test that fowls could be subjected to, and have come out of all these trials still as much praised and as much liked, both by the fancier and general public, as when they were first known. Two things are demanded of the perfect fowl-a large amount of good meat 
when on the table, and a large laying capacity. It is safe to say that no other breed combines these two qualities as well as the Plymouth Rock. There may be hens that will lay more eggs, though we doubt it. Taken weight for weight, we have never seen the fowl that could equal the Plymouth Rocks, and certainly no fowl surpasses them for table use. They are a beautiful breed, combining with their large size, beauty of carriage to a degree not common with fowls. The only fault that can be found with them is the tendency to breed back to show some of the characteristics of the breeds from which they are derived; but those showing only the best. points should be kept for breeding purposes. For the farm no fowl is equal to the Plymouth Rock."

\section{WHITE PLYMOUTH ROCKS.}

The newer varieties of this favorite breed, while unable to usurp the popularity of their older relative, the Barred, have won their way into the hearts of poultrymen as being a general purpose fowl which combines good practical and fancy qual-

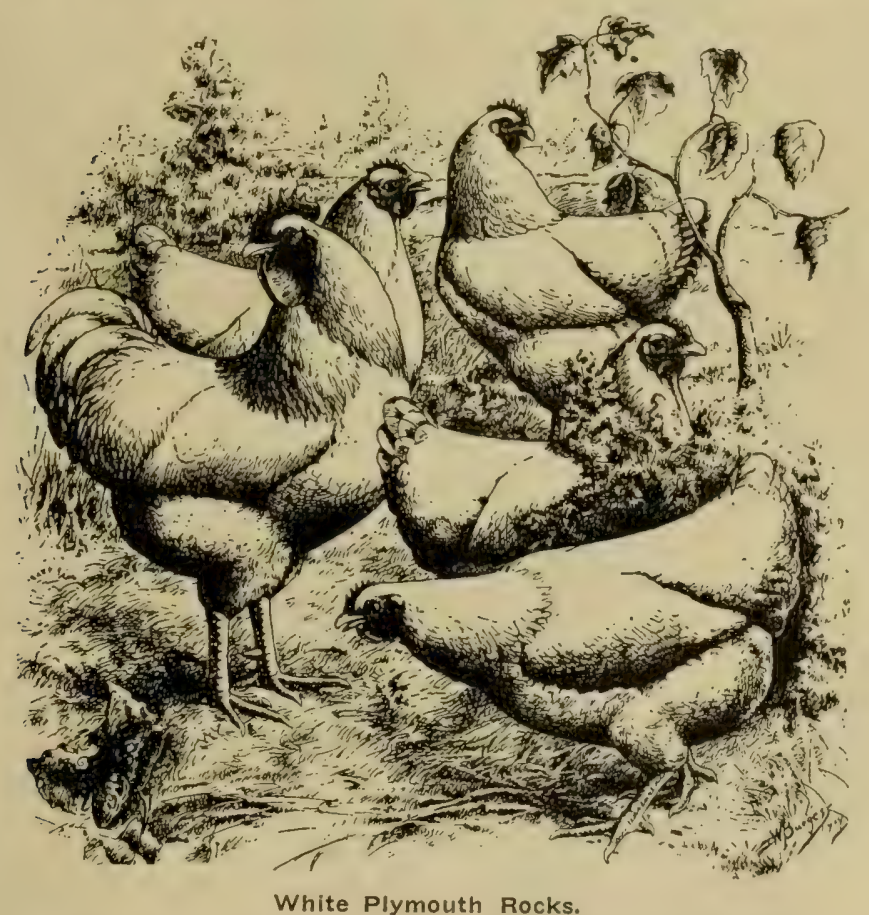

ities. When they made their first appearance there was a great stir among poultry fanciers,' and we are glad to note that the stir has increased with the improvement of the variety. We 
believe there are more White Rocks being bred and sold than there are of any other variety of Plymouth Rocks. There is simply an enormous demand, and price seems to cut little figure so long as the desired variety can be furnished. We believe that the causes for this wide spread popularity may be found in the facts that the Whites are more easily bred true to color, mature more quickly, are good layers, and their white plumage adds one cent more per pound to their value for market poultry over the price paid for colored breeds. The feathers from a well-bred White Plymouth Rock cannot sometimes be told from the feathers of the Embden goose, and the market value of white feathers is higher than that of colored ones.

The White Plymouth Rock is now bred by all up to date breeders, is larger in size than the other Rock varieties and while it does not injure their fancy points nor their egg production, it does improve the quality as a market fowl. We have found the chicks of this variety to be much hardier, quicker to mature, especially so when one considers their remarkable size at maturity. By breeding from selected females that were above standard weight, with good deep breasts, broad backs, short tails and low combs, one can succeed in getting much largersized fowls which lay a larger egg and the egg production will not be injured in any way."

\section{BUFF PLYMOUTH ROCKS.}

This variety has all the good qualities of their Barred and White cousins with the added beauty of the golden buft plumage. A flock of standard Buff Rocks, with their beautiful golden buff plumage, bright yellow legs and beaks makes a beautiful sight and with great utility qualities added to their beauty it is a matter of surprise that they are not more extensively bred in California.

\section{WYANDOTTES.}

Standard weights: Cocks $81 / 2$ lbs.; hens 61/21bs.; cockerels $7^{1 / 2} 1 \mathrm{bs}$. and pullets $51 / 21 \mathrm{ks}$. This breed originated and has beer developed by the American poultry breeder. In size it is about half way betwcen the heavy Dark Brahmas and the Hamburgs the bloor of both these breeds having been used in the earlier strains of the original variety, the Silver Laced. They are ideal as the all purpose fowl both for the table and as producers of gond sized brown eggs.

This breed has a type peculiarly its own. In shape they are all that is best in domestic poultrydom, broad backs, deep well-rounded breasts, set well down to the ground on well developed thighs and stout legs. They are ideal for the small breeder who is compelled to keep his fowls on limited quarters and at the same time some of the largest and most successful extensive poultry plants are stocked with this all-round breed. 
The breeder has a wide range of color markings to choose from in taking up Wyandottes and as they all possess the same qualities as regards utility, it is simply a matter of choice of the color one wants and admires most. In all the varieties in this class one will find plenty of study and interesting matters of breeding to solve aside from the watching and selecting for the utility qualities. To those who admire a closely built, well-rounded plump bird, valuable both for meat and for filling the egg basket we can heartily recommend any variety of the Wyandotte family.

\section{LACED WYANDOTTES.}

There is a peculiar fascination about the production of Laced plumage. Nothing is harder to produce than a specimen carrying true open centered black laced feathers from head

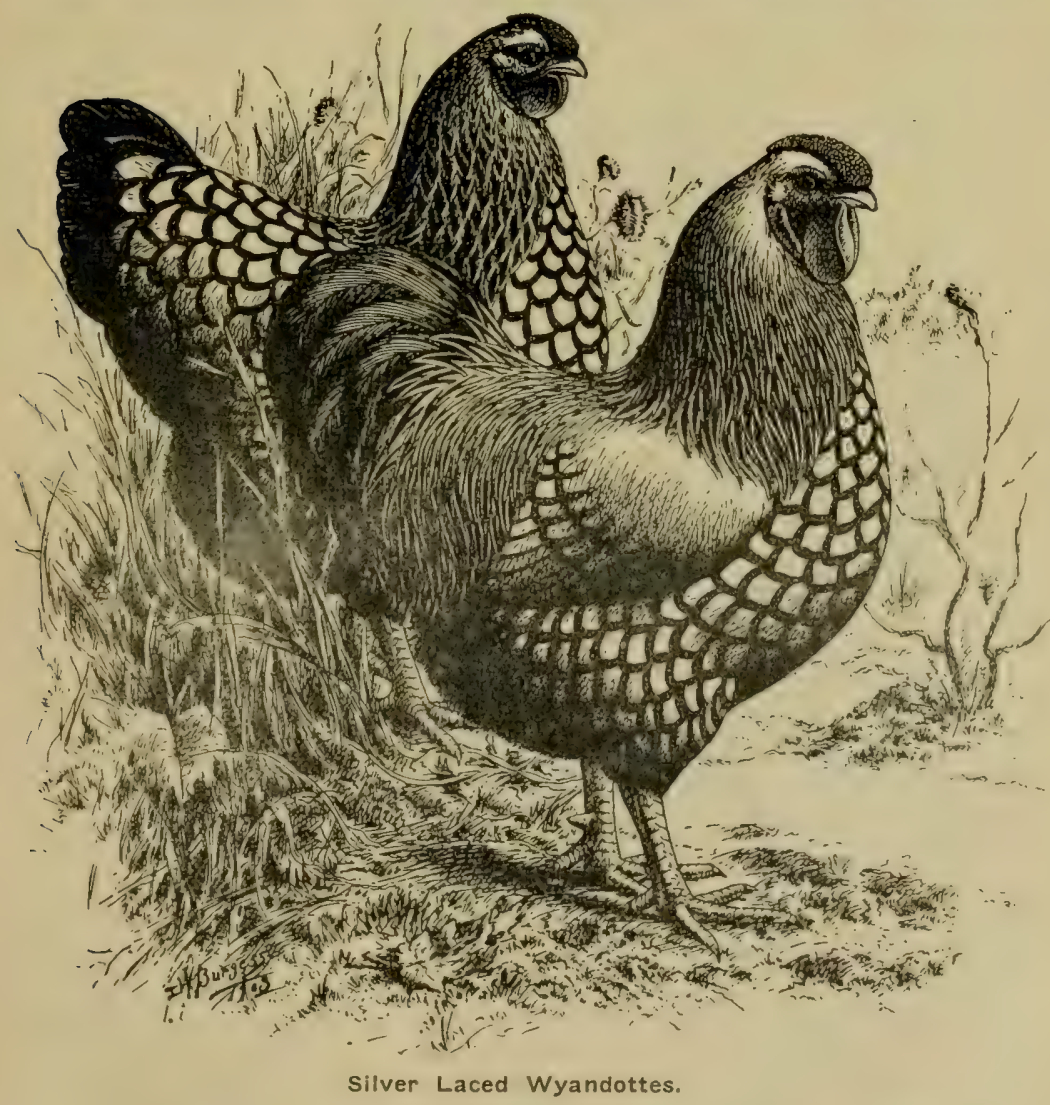

to tail. When we say true we mean absolutely clear open centers. Many birds in both the Silver and Golden Laced Wyan- 
dottes, will look good in the pen, but when examined show a fine black or brown penciling, which later develops into larger lines and destroys the bird as a show specimen or for the breeding pen. Many flocks have been ruined by the use of pullets as breeders that have this defect which is overlooked by the breeder.

There are two recognized varieties in the Laced Wyandotte class, both carrying the same general standard requirement,

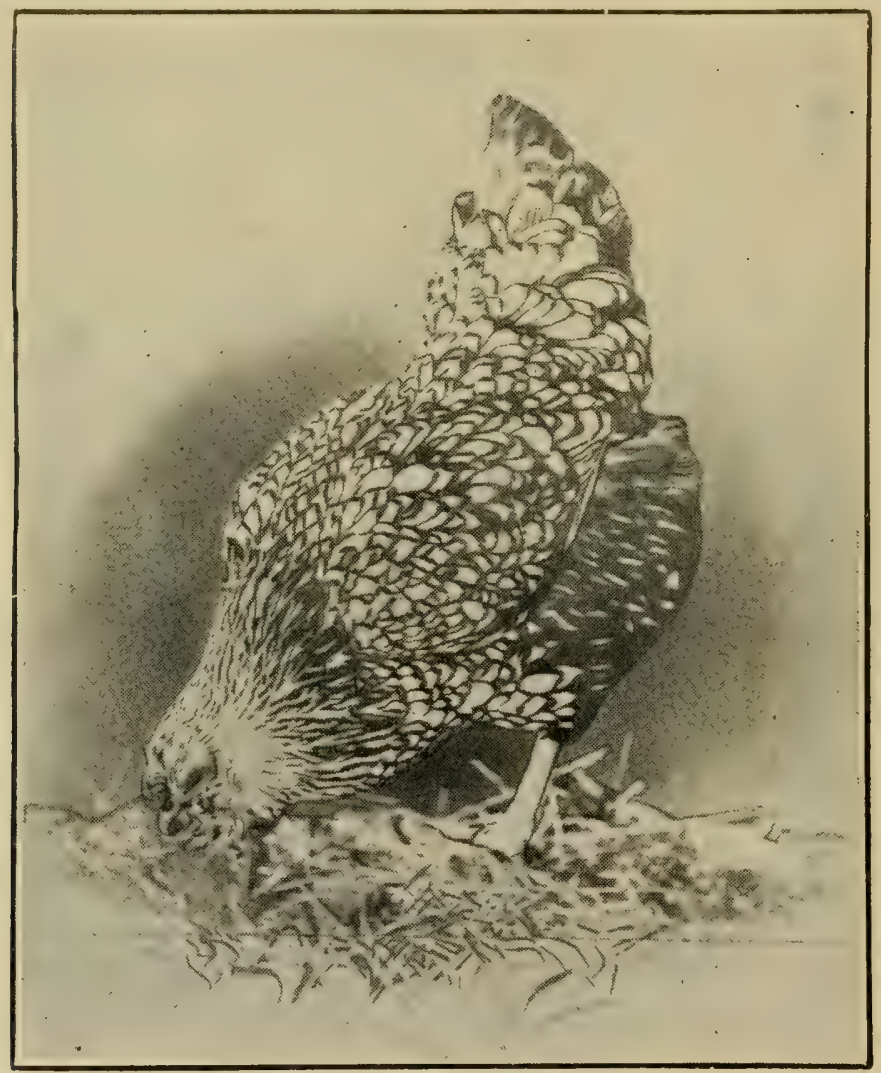

Silver Laced Wyandotte Hen.

with the exception of the color, which in the Silvers is described silvery white laced with lustrous black. In the Goldens, Golden bay is substituted for white. The disqualifications in both varieties are as follows: Solid white ear lobes; combs not rose; shanks any color but yellow, any feather or down on shanks or feet, or evidence of feathers having been removed. Absence of spike in comb. Standard weights are: Cocks, $8 \mathrm{~T} / 2$ 1bs. ; cockerels, $7^{\mathrm{I}} / 2$ lbs.; hens, $6 \mathrm{I} / 2$ lbs.; pullets, $5^{\mathrm{T} / 2}$ lbs. Head should 
be round in appearance, with a broad crown; beak short with nice curve; the comb, rose, lying close to the head, covered with small points terminating in a small spike at the rear; neck should, be short, with flowing hackle in male, and well developed in female; color of plumage should be silvery white (or golden bay) striped in center with black. Back should be very broad, rather short, and well coupled up flat at shoulders with well (leveloped saddle in males (with same color as hackle) and slight cushion in females; breast well developed with a broad, deep appearance; wings, primaries; black, the outer edge with white; secondaries, black with outer half of lower web white coverts, the upper web black, the lower web white with a narrow black stripe along the edge, which widens as it reaches the tip, forming a double bar of laced feathers across the wing. Tail, lustrous black.

Laced Wyandottes are all good layers, and are very superior as table fowls, reaching the fryer age earlier than any other of the American class. Any one wishing to get a bird that combines beauty and utility can make no mistake in taking up these splendid birds.

\section{WHITE WYANDOTTES.}

Every variety of standard poultry has a distinct type which separates it from all other varieties, and can be seen at a glance when once impressed upon the mind's eye. Shape and type of the. Wyandotte family is very marked and distinct from all other types and while many will tell you that a Rock and a Wyandotte look alike except in a matter of comb, there is a marked difference in these two types, and any Wyatidotte breeder will pick out a Wyandotte shape from a pen of Rocks, no matter what kind of a comb the bird is wearing. Of course, there are many so-called Rocks with Wyandotte shapes and perhaps more Wyandottes with Rock shapes. This is especially true with the Wyandottes, as the male birds seem to have a tendency to show the longer back and length of legs and shanks of the Rocks, and as back and legs make up a large part of the type form of the Wyandotte this is a bad defect. The true Wyandotte should be a bird carrying a broad crown, medium length of neck, well coupled up with the shoulders, a back not too long from the shoulder to center of back, with a cushion just between the Cochin and the Rock, a tail not too long in feather, well spread at the base and not showing the pinched appearance of the heavily feathered Cochin; fluff should be more abundant than on the Rock, and should give the howk and upper shank the appearance of being rather short and well rounded out, the breast should be full and deep, running down straight from: the wattles and giving the bird the appearance of a full-breasted, well-rounded-out specimen, the shanks or 
lower legs shuuld be set well back on the bird, and not too long. The whole symmetry of the Wyandotte should be that of a plump, well-rounded-out and nut too closely feathered bird. The American standard for White Wyandotte is in substance as follows: In males: The head should be round, short and rather broad, and snow white in color. The beak should be

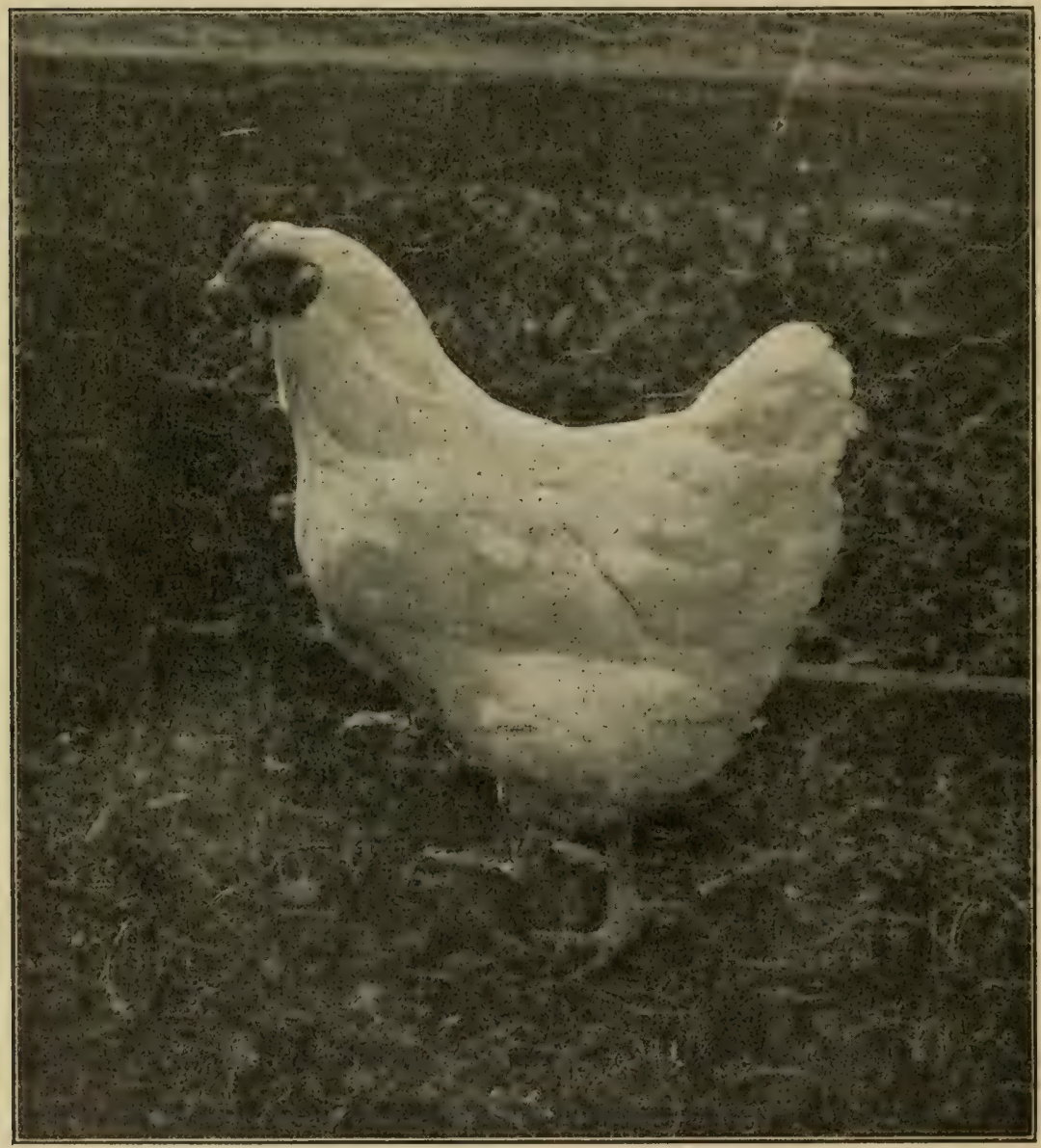

California Bred White Wyandotte Pullet.

well curved and stout, in color a bright yellow. Wattles medium in length, fine in texture, well-rounded. Ear-lobes, well developed, smooth and bright red in color. Neck should be short, well arched with abundant flowing hackle. Back short, broad and flat at the shoulders. Saddle bruad, full and rising with a concave sweep to the tail. Breast, broad, deep and fully de- 
veloped. Fluff full feathered and well rounded. Tail carried fairly upright, sickles of medium length, gracefully curved over the tail, the ends projecting slightly. Legs and toes-Thighs short and well spread, covered with soft feathers. Shanks rather short and stout, free from stubs and feathers.

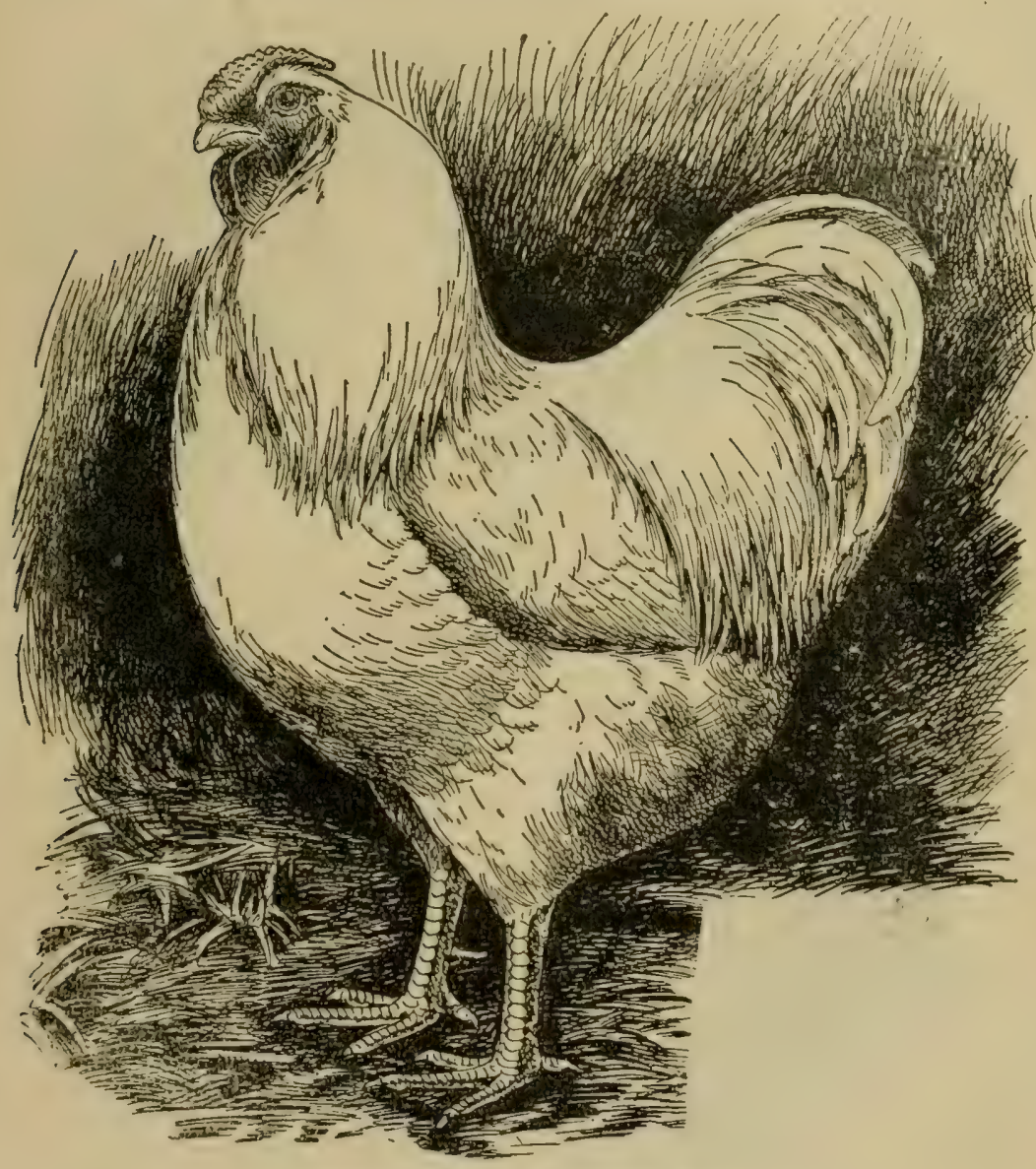

Standard White Wyandotte Male.

In females: The shape description is practically the same. The tail in females is described as well developed, well spread at the base and carried less upright than in the male. The color in both male and female should be white thruughout, including the quill, which should be as white as the wet of the feather. The color of eye in both male and female shuuld be a bright bay, and this section should be carefully watched, as 
many strains of White Wyandottes throw that is known as light eyes, and a good eye materially helps the appearance of the bird.

Disqualification of White Wyandottes: Solid white in ear-lobes; combs other than rose or falling over to one side; or so large as to obstruct the sight; shanks other than yellow in color; any stubs or feathers on shanks or feet or unmistakable

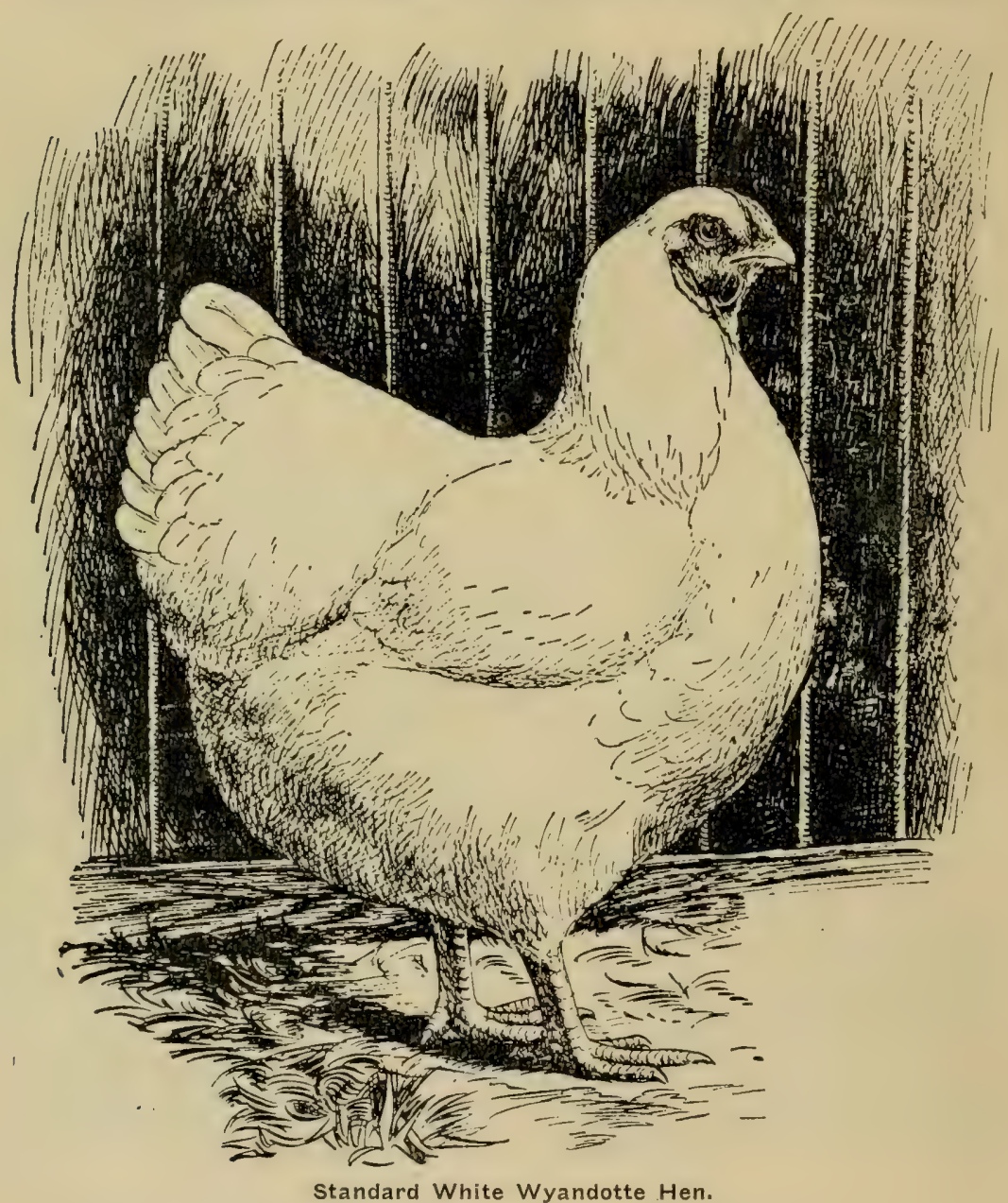

indications of feathers having been plucked from same. Absence of spike in comb.

There are three vital points to be considered in the breeding of White Wyandottes, first to get the proper tvpe which must come largely from the hen, second to get the pure white plumage which must be obtained to a large degree through 
the male, and third to get the low, well set properly pebbled comb. The comb should not be too large yet should coveI the head and lay close to the crown and back of the head with a good medium length spike. This spike. should be in proportion to the size of the pebbles on the comb, and should go to make up the well-rounded-out bird. If these three points are followed in breeding, the result will be a well balanced flock of good youngsters. The utility qualities of the White Wyandottes are too well known for us to add much to their already splendid reputation as the ideal money maker of American poultry culture. White Wyandottes are especially adapted to the California climate, and are each year growing in favor with California breeders. No breeder can make a mistake in taking up this ideal broiler and egg producing variety.

\section{BUFF WYANDOTTES.}

This member of the Wyandotte family is having quite a run in Californiä and are proving great favorites with all who breed them. They combine attractiveness with great pro. ducing qualities and like the other members of this popular family are hardy and fast growers.

In general type the Buff's are like the other Wyandottes and in color are a beautiful golden buff in all sections.

\section{PARTRIDGE WYANDOTTES.}

In no other branch of live stock husbandry is the demand for a combination of pleasing appearance and utility so marked as in the breeding of standard poultry. The so-called "fancier" has not only developed the fancy points of modern domestic poultry. but is responsible for their increased producing qualities as well. The fancier must have a bird of beauty in order that he may have an incentive to improve and perfect them; at the same time, the modern fancier is intensely practical, and a variety with only beauty to recommend it no longer interests him. It is this practical trait that has brought out the many new varieties with money-making qualities, carrying the color and markings of some of the older varieties that were beautiful in appearance, but lacking in utility qualities. A case in point is the subject of this article-the Partridge Wyandotte. No more beautiful bird was ever produced than the grand old Partridge Cochin, but with their massive size and over-abundance of feathers, they were neither profitable as an egg fowl, or for table purposes. Naturally their color was much admired by the American fancier, and in order to retain this color it has been produced on the type and make-up of the all-purpose Wyandotte, the result being the Partridge Wyandotte of today.

Of the true Wyandotte type, they are, like the rest of that 
family, an all-purpose fowl, averaging from I 50 to 185 eggs per year, and producing a large part of these during the winter months. They are splendid table fowls, fast growers, and quick featherers. It is on color that their admirers especially recommend them for the California climate. In this country of everlasting sunshine the Partridge Wyandotte will come through the long spring and early summer to the moulting time without apparently showing the effects of the rays of the sun on their plumage. This undoubtedly proves that they are adapted to this climate, and is a good point in their favor, both from a utility and fancy point of view. Their beauty is unquestioned, even by those who do not breed them, and have their favorites in other varieties. The females are of especial beauty, with
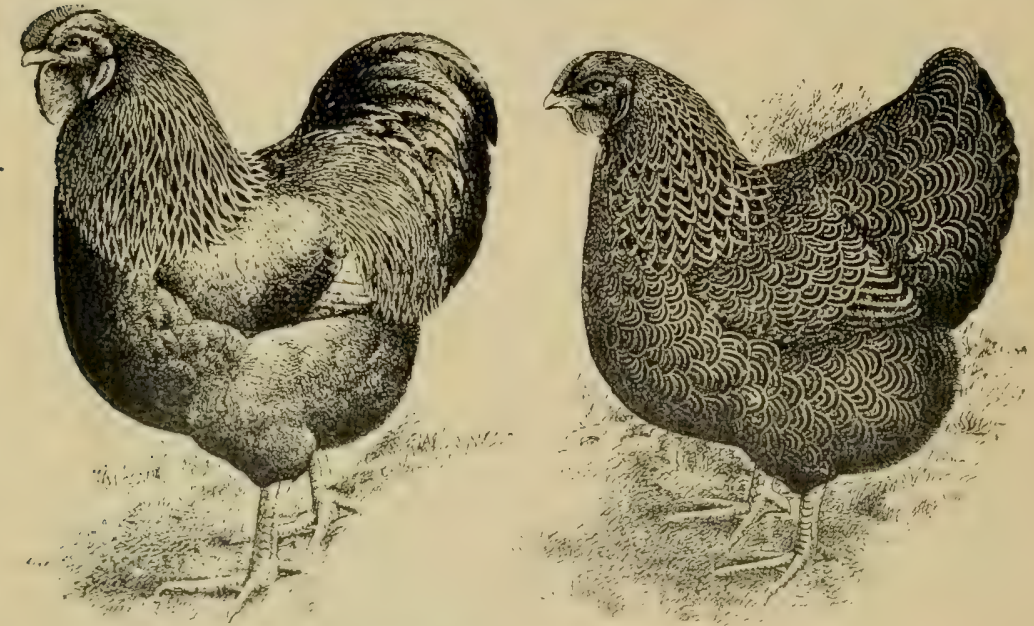

Partridge Wyandottes.

black penciling on a ground color of mahognay red; the males with rich red hackles and saddles, striped down the center with metallic black stripes, also make a striking appearance. The Wyandotte shape is sufficiently striking in itself to attract admiration from poultry lovers, and when clothed in this magnificent plumage, it makes a fancier's bird par excellence.

The disqualifications are: Ear lobes more than onehalf positive white; shanks other than yellow or dusky yellow in color. Any feather or feathers on shanks, feet or toes are unmistakable indications of feathers having been plucked from same. Combs without spike; wry tails and crooked backs. The males should weigh, cock $8 \mathrm{r} / 2$ pounds, cockerel $71 / 2$ pounds; females, hens $61 / 2$ pounds and pullets $5 \mathrm{~T} / 2$ pounds. Thestriking color points in the male are a low comb, hackles rich red, with black stripes through center of each feather, 
tapering to a point near the end of the feather, but not running into the red; saddle and back of same color; wings should have a well defined bar of black across the coverts, when wings are folded; body, breast and tail should be glossy black; undercolor in all sections dark slate. The color of the female is hackles red, several shades lighter than the male, striped the same as the male, with the exception that penciling is allowed in the central portion of the feathers. The back, breast and wings, mahogany red or reddish brown, distinctly penciled with black; the penciling to conform to the shape of feathers; undercolor dark slate. The tail, black or brownish black, the two highest main tail feathers penciled as described in other sections.

\section{SILVER PENCILED WYANDOTTES.}

This variety is identical with the Partridge in all respects except color of plumage. In markings the silvers are silvery white and black instead of Mahogany and black as found in the Partridge. The Silver Penciled have made some wonderful egg records and by many who have been giving their attention to them the past few years, are considered the best layers of all the purpose varieties. No prettier bird can be found and the future for this variety is very promising.

\section{COLUMBIAN WYANDOTTES.}

This is the latest addition to the Wyandotte family and have only recently been admitted to the standard. They have been carefully bred however for the past ten years by some of

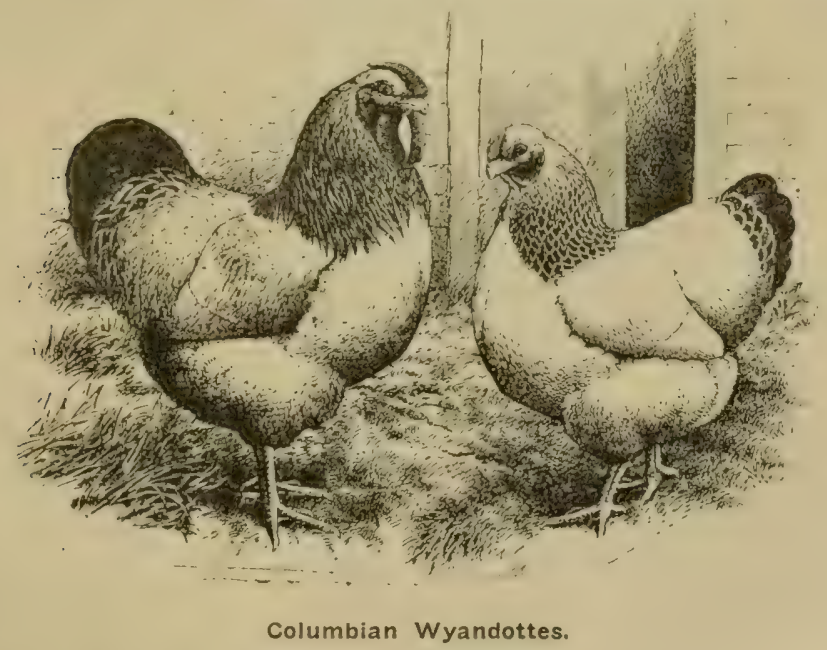

the best breeders in the country and are now on a very firm basis from a breeders standpoint. 
The Columbians are the true Wyandotte shape and in color are the same as the Light Brahma.

That the Columbian Wyandotte has come to stay is attested by their great popularity, and the immense demand for stock and eggs. They are certainly handsome, fine table poultry and splendid layers, and seem to have as much if not more merit as a first-class all-round fowl as has any breed in America.

\section{ORPINGTONS.}

Standard weights: Cocks, ro lbs.; cockerels, $8 \mathrm{I} / 2 \mathrm{lbs}$; hens, 8 lbs.; pullets, 7 lbs.

The Orpington type is a broad and massive body set on nice short legs, and this type should be insisted upon, for this, combined with white skin of the finest texture and its white flesh and legs, make it the table bird par excellence. One could scarcely understand the great difference that exists between such a bird and one that had been bred regardless of table qualities; it is a pleasure to fatten and dress them, and when it comes to the eating-well, there is all the difference in the world.

The belief existed for many years, and does still in the minds and many today, that to produce an ideal table fowl the laying qualities have to be sacrificed. This, no doubt, was to a certain extent quite true, but careful selection has upset all this, and today there are flocks of Orpingtons that can beat the lighter varieties that are bred for eggs alone. Another excellent feature is that the Orpingtons do most of their laying during the season when eggs are scarce.

Birds bred any time between the months of December and May will, with ordinary care, pay a good interest on capital expended. The cockerels make excellent broilers and as fatted soft roasters they do equally well.

\section{BUFF ORPINGTONS.}

The first pair of Buff Orpingtons ever seen at a Poultry Show were exhibited at the Dairy Show, Islington, London, in October, 1894. Ever since that date this famous breed has never looked back and its supporters can be numbered by thousands. The merits and utility of the breed make it one of the most attractive and useful of all classes of poultry, combining the beautiful, popular buff color with admirable table and laying qualities. We think it cannot be disputed that the Buff Orpington comes as near to the ideal fowl as it is possible to gret one. We have bred most kinds of chickens but of them all the Buff Orpington has the warmest place in our hearts. 'The color is so pleasing to the eye and the carriage and symmetry are a joy to behold; added to this a more than usual intelligence and gentleness, makes it a pleasure to attend them. Feather and shape alone will not win praise from the real fancier, for he will 
ask, "Do they lay well, and how are they for eating purposes." The answer to these questions is what determines the lasting. popularity for a breed or variety. When eggs are scarce and most of the feathered world are taking the rest that nature has mapped out for them, you will find Mrs. Buff Orpington busy and doing her bit for the egg basket. Often she will lay ican through the moult and come out the other end just as fresh and rosy and enter the new year with a zest that would clo justice to a yearling.

As a table bird the Orpington is only equalled by the Games and Dorkings families; their delicious, finely graned hesh, firm and succulent and affording large cuts off the long, deep breist, make them, with their white legs, a joy to the epicure. The cockerels can be brought to a nice plump condition at any age, if ordinary care is given them and they look so much nicer than the thin, yellow-looking specimens of chickens one sees so often at the dealers.

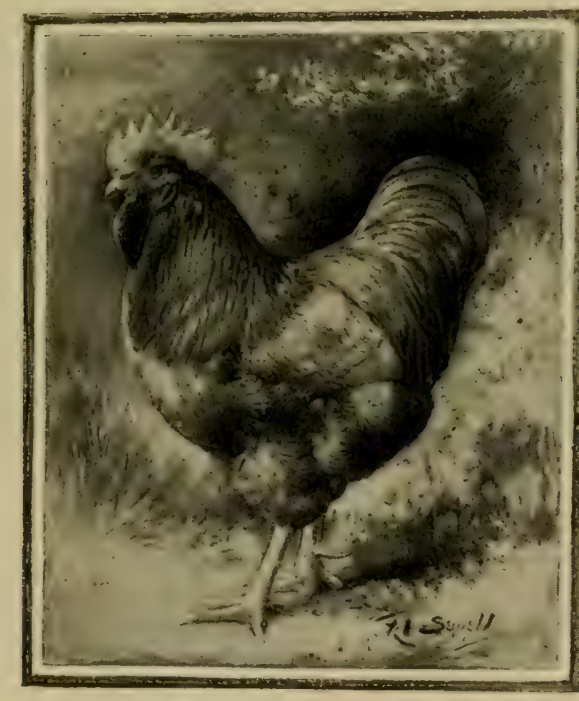

Buff Orpington Male.

As a fancier's bird they are ideal. Being rather difficult to breed true, good specimens always bring good prices, and we feel safe in saying that this will hold good for many years to come. The following is our conception of an ideal bird;

Male: A small, neat head, nice and full over the eye, the comb of medium size, evenly serrated, five points preferred and to be free from side sprigs and well set on the head, slightly following the curve of the neck. A full, bright intelligent eye which should have a medium sized black retina and the iris of an orange 
red; wattles nicely rounded; ear lobes medium size and fairly long, a gracefully curved neck and full hackle. The breast should be broad, deep and full. carried well forward and have a long straight breast bone. Short back with very broad shuulders, the saddle raising slightly with a good flowing hachle. Wings should be strong and carried close up; the skin white and thin and of a fine texture, with firm, white flesh; medium tail flowing and slightly inclined backwards, short thighs, shurt, thick, clean white shanks, the less pink the better as this takes away from the beauty of the bird; four thick, short toes, well and evenly spread. He should be colby, graceful and upright, weight nine or ten pounds when mature. Plumage close and hroad, strung feathers. In color the male may be any shade of buff from lemon tu a deep rich buff, avoid in the former paleness and the latter redness. The color should be perfectly uniform

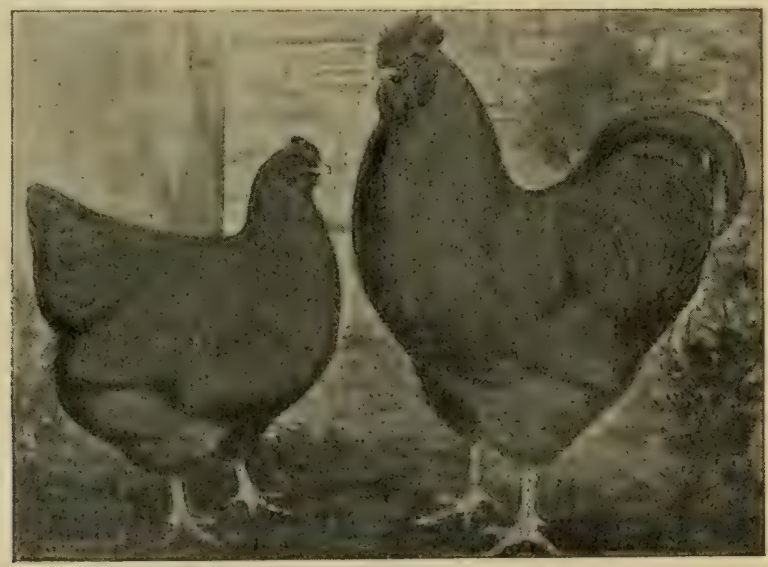

Buff Orpingtons.

throughout allowing for a greater lustre on the hackle and saddle feathers and the wing bow. The comb, face ear lobes and wattles should be bright red.

With regard to the females their characteristics should be verv similar to the male, with a small cushion giving the back a nicely curved appearance, medium tail inclined backward and upward; weight seven to eight pounds when mature. In culor they shuuld be even all over with no white in hackle or flight and as much buff undercolor as possible.

The beginner must not be discouraged if he cannot get birds as abnve described to start a breeding pen with for they are few and far between. We helieve there is a great future for the Buff Orpington and know of no better breed to recommend the beginner to take up for the demand is enormous and will be greater as the real qualities become better known. 


\section{WHITE ORPINGTONS.}

The White Orpington is a variety that has recently come very much to the front. Their origin can be traced to several quarters; Mr. Cook claims to have made the variety by crossing White Leghorn cocks to Black Hamburg pullets and the pullets from this cross mated to White Dorking cocks, but we venture to say that nine-tenths of the White Orpington stock of today could be traced to the new Sussex fowl, which are known as

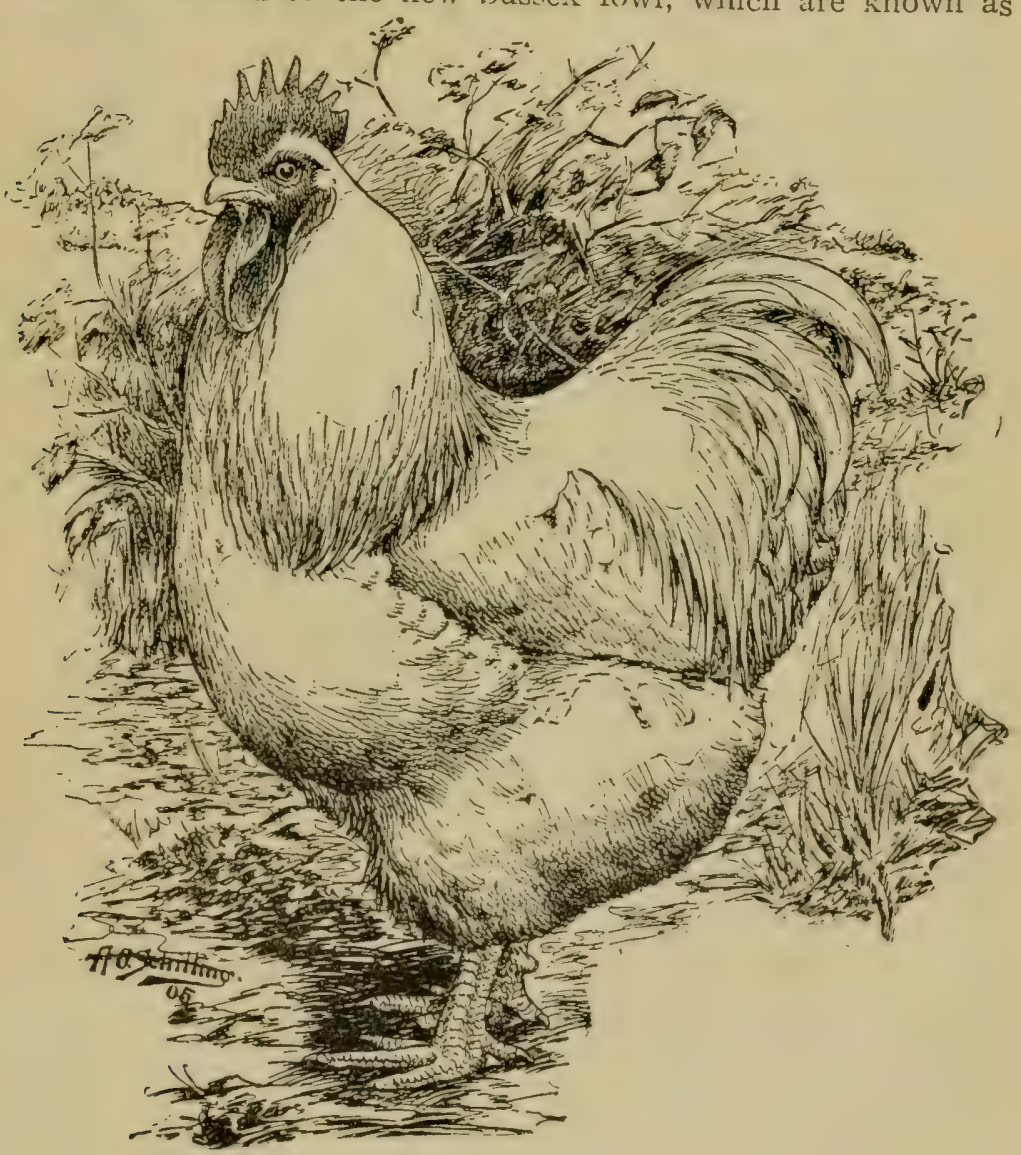

Standard White Orpington cock.

Albions. For the develupment of this breed there is none deserving of as much credit as Mr. Godfrey Shaw. This gentleman has worked hard and patiently in their behalf. The Albion in brief is as follows: plumage all white, white beak, white legs, red face, ear lobes, wattles and comb. The shape is the only thing that differs from the White Orpington, which is rather long, much re- 
sembling the Dorking. It will be seen that it would take but little trouble in selection to breed the Orpington shape to these birds, which of course, has been done. Probably there is no breed living that does as well in the fatting coop as the White Orpington. There are a few first crosses that may be preferred, but as a straight breed, the White Orpingtons are most excel-. lent. This we attribute to the fact of their having been bred for that purpose almost exclusively in Sussex.

After breeding the White Orpingtons for a number of years we have no hesitancy in saying that they are one of the best layers in all-purpose classes. Their eggs are of extra size and many of our hens have been absolutely non-setters and quick growers. Smallness of bone is a desirable feature, for this

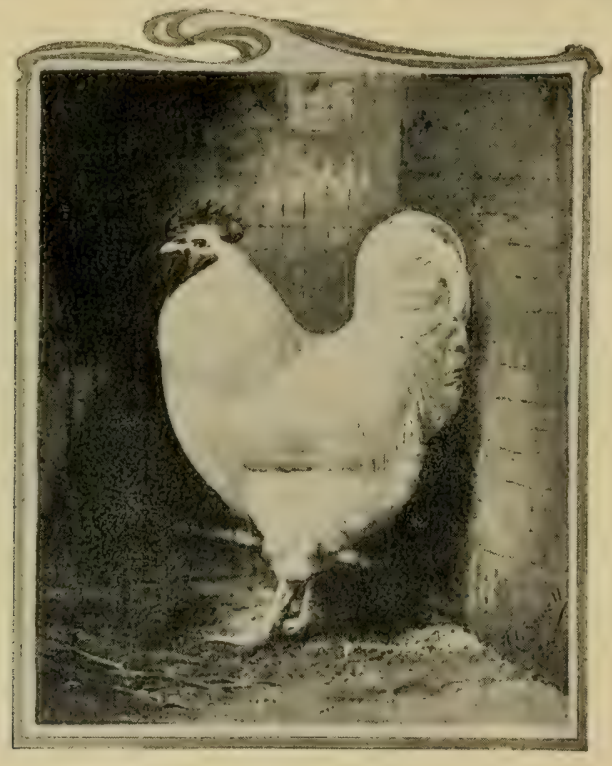

White Orpington Cock.

of course makes the ideal table fowl. We have seen a cock that weighed about twelve and a half pounds, but ten is nearer the average. We trust the size of this breed will be kept where it is, for greater size would have a tendency to destroy the quality as a table fowl. To those breeders who love a white chicken, the White Orpington will well repay an investment, for thev are a pleasure to the eye and have such' good utility qualities. The chief difficulties to overcome in breeding exhibition specimens is shape and color. A nice, short back is hard to get accompanied by good depth of body. In the male brassiness is giving trouble, and those that get there first with all white, good shaped birds will find quite nice figures awaiting them. In 
selection for breeder, type must be always borne in mind. A broad, deep breast, compact, massive and solid body and set low on nice, short legs. Plumage should be close and not carry too much fluff. All long backed and long legged specimens must be discarded.

\section{BLACK ORPINGTONS.}

This variety of the Orpington fowl being the earliest introduced is in consequence the most established as to color and type. As is generally known they were produced by crossing a large Minorca cock to Black Plymouth Rock sport pullets; the pullets of this cross were then mated to a clean legged Langshan male, and the offspring carefully bred to large, deep-bodied, short legged birds. It is also beyond doubt that other methods were used to produce the Black Orpington, and we personally have

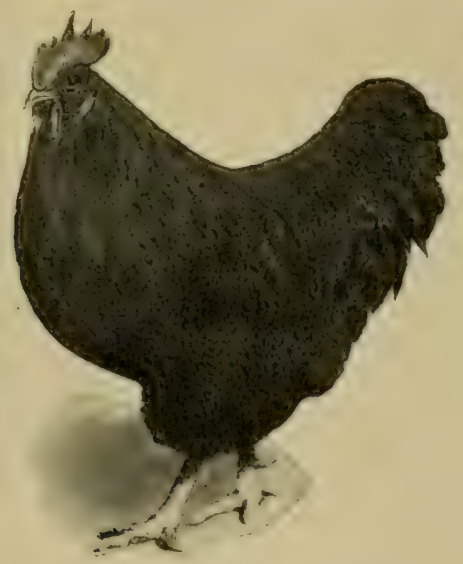

Black Orpington Male.

met two breeders who claim to have none of the original blood in their birds whatever. Be this as it may, all that one can say is that the originators are to be congratulated on giving to the world such a splendid specimen of the poultry kingdom, for a more noble bird it will be hard to find. On the utility side they have a grand record. In open competition they have beaten both the Mediterranean and heavy breeds, and always make a name for themselves wherever shown. As an instance of this we would call our readers' attention to what has been done by this breed in Australia where it is safe to say the Black Orpington is first favorite. As a table bird they are very good indeed, the only detriment being their black shanks, which however, we find does not count so much in America as in Europe. For the size of the breed the Black Orpingtons are very active and if 
allowed to range will travel far from home in search of food. They are small eaters, will fatten readily if cooped and fed well. The eggs of a mature specimen will average seven to the pound. For the city lot they are an excellent variety to keep; not showing the dirt like the lighter colors, they always look fresh and clean, and are a joy to behold. Few things look as beautiful as the Black Orpingtons when in condition. In breeding Black Orpingtons, to get good size the female should be fully matured, nice large hens, but not coarse in bone, carrying as much green sheen as possible. If the male lacks good color it is most important that the hen should be strong in this point, it being useless to try and breed from male and females that show much purple. A good mating will insure the breeder getting good males and females and it is not at all necessary to run two breeding pens to produce show birds of both sexes.

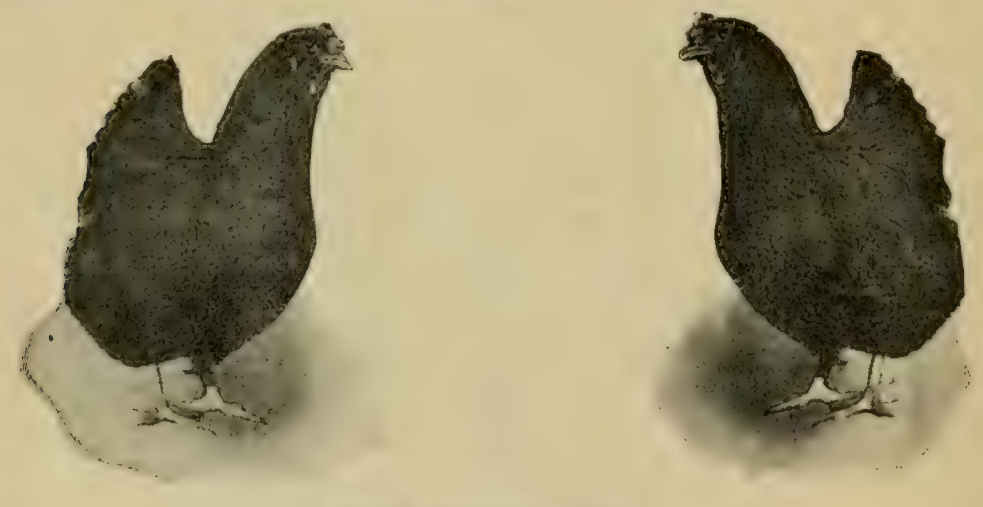

Black Orpington Hens.

In general type the Blacks are the same as the Buff and White varieties. In color of plumage they should be glossy, metallic black, with greenish sheen. Purple barring should be avoided and is one of the main color defects found in this variety.

\section{RHODE ISLAND REDS.}

Known as the "farmer's fowl" and from the time of their origination bred along lines calculated to bring them to the highest standard of commercial excellence, the Rhode Island Reds have become one of our most popular all-purpose fowls, not only with the commercial breeder but with the fancier as well. They enjoy the distinction of being the only American variety named for a locality, and developed in the place which gave its name. Another unique distinction of the Reds is that the fancier had no part in their "making." They were produced and bred for years in the locality where they originated, becoming practically the common fowl of that district of Rhode Island, before 
they were recognized by the fanciers and the work of molding them into shape as to type and color commenced.

According to the best authority, their history dates back for more than fifty years. It is common knowledge among the older breeders that the Buff Wyandottes and Buff Plymouth Rocks owe much of their early make-up to these red fowls from Rhode Island.

The Exhibition Rhode Island Red of today is a much finer and more finished fowl than the Reds bred on the farms around Little Compton, Rhode Island thirty or even fifteen years ago

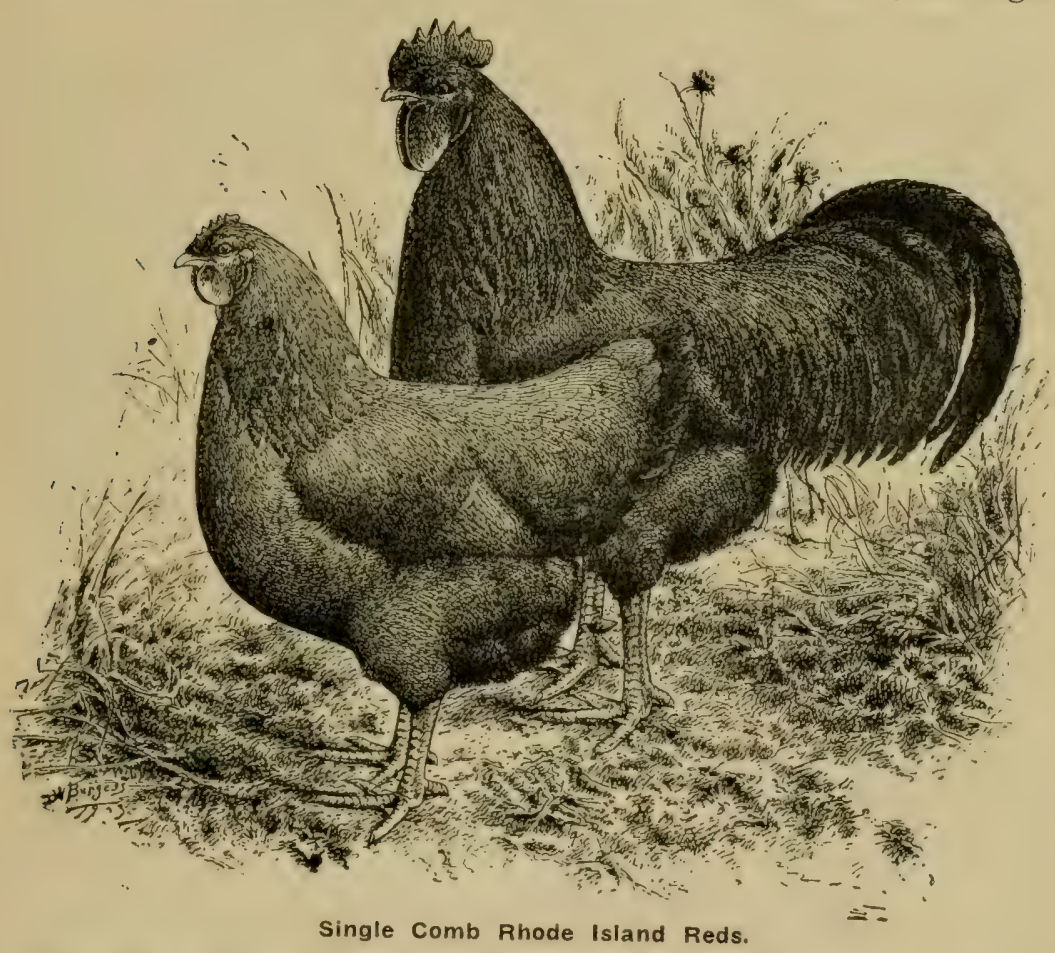

and have made great improvements over the first Reds exhibited. At the same time, all of the good qualities of the earlier birds have been retained and they are today as they were fifty years ago on the Rhode Island farms, great producers of eggs and the fastest growers of any of the middle weight varieties. In fact no other birds fit in between the small Mediterranean and the middle class American' breeds as does this plump active fowl.

Several of the best breeders on the Pacific Coast were quick to see the great possibilities in these useful birds and have developed them along their original lines of utility and beaty 
qualities, until today the Coast can boast of some of the best Reds in the United States.

The standard weights are for cocks, 8 $1 / 2$ 1bs.; cockerels, $7 \mathrm{I} / 2$ lbs.; hens, $6 \frac{1}{2}$ lbs.; pullets, 5 lbs. The disqualifications are, ear-lobes more than one-half white; one or more white feathers in outer plumage; shanks other than yellow or reddish horn. Their chief characteristics, as given in the standard, are, red color throughout, except black in the tail and wings of both sex; oblong shape bodies compact form and smooth surface plumage.

\section{BREEDS FOR MEAT PURPOSES.}

In this class we find breeds that are in the all-purpose class and others that have little to recommend them outside of their value for fattening and turning into dressed stock. In the English class we find the Orpingtons and the Dorkings, two of the best meat breeds we have, and in the case of the Orpingtons especially adapted to California conditions. Both these breeds are good egg producers and profitable outside of their meat qualities. In the French class we find the Houdans with good egg records and splendid table qualities while in the same class are the Crevecoeurs and La Fleche (these two very little bred in California and not particularly adapted to this climate) wit 1 little to recommend them besides their meat qualities.

In the Indian Game we have a bird of not overly great egg producing qualities but one of the best as a table fowl and as a fancier's bird.

The question of producing dressed poultry for the California markets has become a very prominent feature with California poultry breeders the past few years and therefore the matter of selecting a breed that will fill the bill for this purpose is an important matter to decide. With the many large tourist hotels in Southern California which demand the highest class of goods and are not particular as to the price they pay so long as the quality is right the selling of strictly first-class dressed poultry has become one of the most paying lines in the business. In order to furnish the quality demanded the breeder must have the breeds that have been especially selected and bred for meat purposes. We have given this branch of the business much thought and practical demonstration and can most heartily recommend it to those who have a liking for the strictly meat branch of the business or for those who wish to combine it with the other branches:

\section{ORPINGTONS.}

After a series of very careful and thorough experiments with the different breeds as regards their value as dressed stock we are convinced that the Orpington family is without a peer for this purpose. This breed will put on two pounds of flesh 
under a forcing food while any of the other breeds are putting on one pound. The quality of the flesh is also superior to all the other meat breeds and brings a good advance in price in a market where quality is recognized. The general characteristics and all-round good qualities were covered in another part of this chapter under the head of general purpose breeds.

\section{DORKINGS.}

For years the Dorkings held the main place in England as the table fowl. Since the advent of the Orpingtons they have had to share this honor with them but still hold a firm place with the English poultry breeders. Dorkings have never been extensively bred in California but those that have been bred here have given good results. They are said to be the oldest fowl we have and some claim that their line can be traced back to the time of the Ark. The standard varieties in this class are White, Silver-Gray and Colored. All the Dorkings have low-set rectangular shaped bodies with deep keels and short legs. Their skin and flesh are white. The Silver and Colored Dorkings have a single comb and the Whites a rose comb. All three varieties have five toes. In color the Silver-Grays are a combination of black and grayish white while the Colored variety are a combination of black, light straw and slate color.

\section{THE FRENCH BREEDS.}

Since the French people are noted for their skill in pruducing and preparing poultry for the market it is natural that we should find some of the best meat breeds produced on French soil.

\section{HOUDANS.}

Houdans are to France what the Dorkings and Orpingtons are to England. An American poultry breeder who recently made an extended visit to France reports that in all the large French markets at least two thirds of all the birds sold are Houdans. Houdans are great egg producers as well as a fine table fowl, and it is a matter of surprise that they have not been more extensively bred in this country. The Standard calls the Houdan plumage mottled black and white, the white on tip of black feathers in proportion of about one tipped feather to three solid black ones. The crest should be round and not divided at the top. In type the Houdan resembles the Dorking. Toes, five upon each foot, straight except the fifth, which should be detached from the others and curved upward. Standard weights: Cocks 7 lbs.; cockerels $6 \mathrm{lbs}$; ; hens $6 \mathrm{lbs}$. and pullets $5 \mathrm{lbs}$. 


\section{CREVECOEURS AND LA FLECHE.}

Both these breeds are popular as table fowls in French, English and American markets. Their plumage is rich glossy black. The Crevecoeurs have large crests and beard and their standard weights are: Cocks 8 lbs.; cockerels 7 lbs.; hens 7 lbs.; pullets $6 \mathrm{lbs}$. The standard weights for La Fleche is one-half pound additional to those called for in the Crevecoeurs.

\section{GAMES.}

All the varieties of Games are known the world over as great table forwls. A well bred Game is high stationed, closely feathered and carrying an abundance of breast flesh. With the exception of the Indian Games they are not a large bird but dress up in better style than some of the larger birds.

\section{CORNISH AND WHITE INDIAN GAMES.}

By many the Cornish Indians are considered the best of all table birds. A bird of good size closely feathered with a massive breast and the best of flesh. Certainly the Cornish

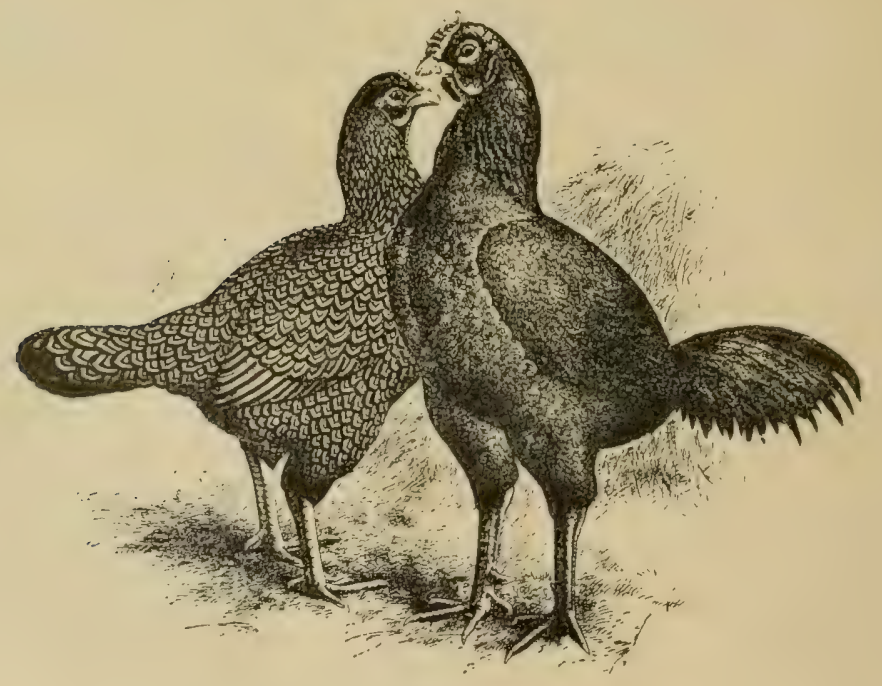

Cornish Indian Games.

Indians have a just claim for first honors as a table fowl. The standard weights for both the Cornish and the White Indians are: Cocks 9 lbs.; cockerels 7 I-2 lbs.; hens 6 I-2 lbs.; pullets $5 \mathrm{I}-2 \mathrm{lbs}$. 


\section{BLACK BREASTED RED AND BROWN RED GAMES.}

These as well as all the Games crosses are excellent table birds, fairly quick growers and are hardy and easy to raise.

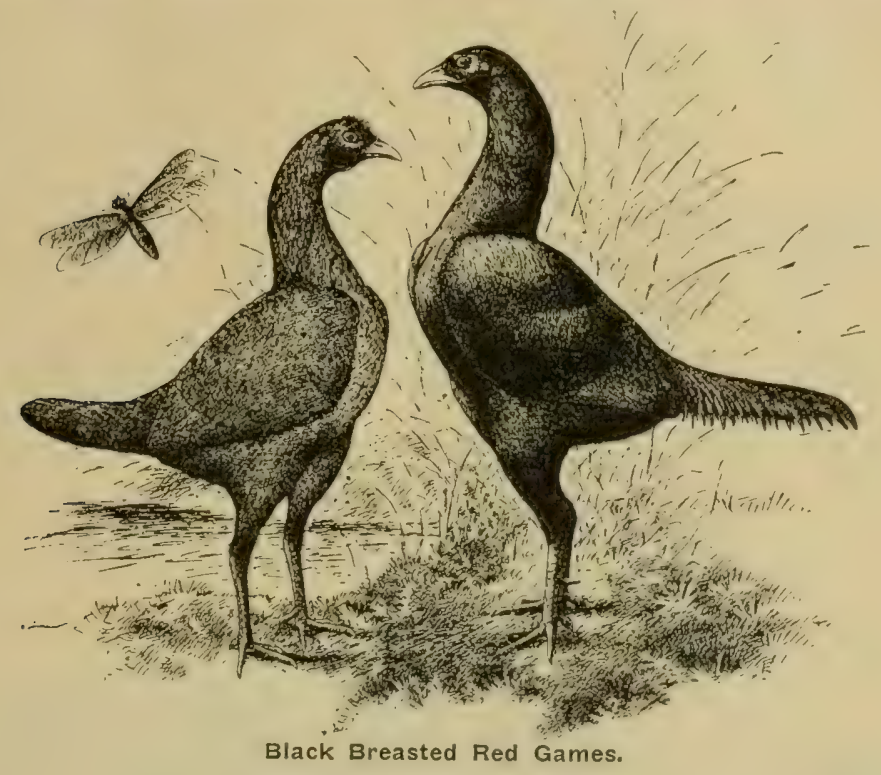

THE ASIATIC CLASS.

This class is made up of all the heavy weight varieties in the Tpoultry world, comprising the Light and Dark Brahmas, Buff, Partridge, White and Black Cochins and Black and White Langshans. While these large birds are better suited for the colder climates of New England than the tropical climate of California there are sections of this State that are especially suited to their production. In our high mountain valleys where zero weather is found the Brahmas and Cochins are at their best. In the case of the Langshans any section of the state is suited to their breeding, for while they are a large bird they are closely feathered and will do well in any climate. The varieties in this class, with the exception of the Langshans and Light Brahmas, are not noted as egg producers but they are the greatest of all breeds for the large suft roaster and make ideal capons. The Brahmas are the fowls mostly used in the great "roaster" markets at Philadelphia and Boston.

\section{COCHINS.}

The Cochin type is a deep bodied, massive bird all curves and rounded out lines; a bird with rather stately carriage, leaning slightly forward; slow in movement. They are a bird of 
profuse feathering, their plumage being loose and giving the appearance of even a larger bird than they really are. There are four varieties in this breed, Buff, Partridge, White and Black. The two latter are very little bred but the Buff and Partridge are popular the world over. The standard weight for Cochins is, cocks I I lbs, ; cockerels 9 lbs.; hens 8 I-2 lbs.; pullets 7 lbs.

\section{BUFF COCHINS.}

The oldest of our buff varieties and bred to great prefection in color qualities. They are fair layers and make excellent soft roasters.

\section{PARTRIDGE COCHINS.}

Here is one of the most beautiful of the standard varieties and has been bred to a high state of prefection by the American

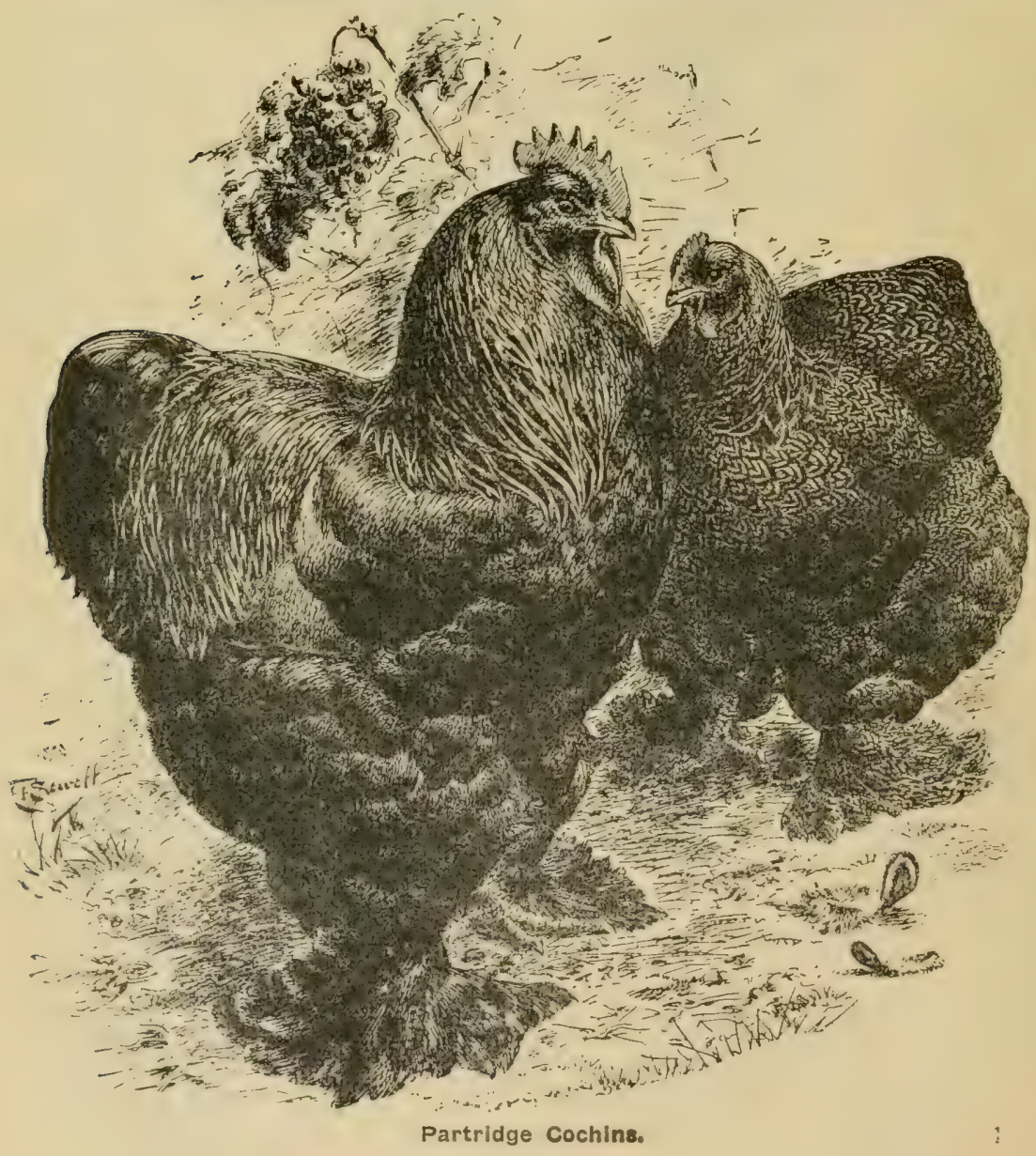


and British fanciers. Their color is the same as described in the chapter under the heading of Partridge Wyandottes. They are excellent winter layers and make extra fine roasters.

\section{LIGHT BRAHMAS.}

The most interesting of all the varieties in point of history, the largest of the poultry breeds, the grandest in appearance of all poultrydom, are some of the interesting points of the noble Light Brahmas.

The early history of this variety is notable in that it made famous, and in turn was made famous by the two fathers of standard poultry culture in the United States, Mr. Philander Williams and Mr. I. K. Felch, who are both enjoying a good old age, still interested in ther first love.

Since its first introduction in America the Light Brahma has been a popular fowl. Many breeds and varieties have come and gone, but the old Light Brahma remains, the love and pride of a host of fanciers and the mainstay of a large number of market poultrymen.

As an exhibition fowl, the Light Brahma occupies a prominent place. This large, massive bird, beautiful in its color markings of pure white and black; is very attractive in the pen. In New England its popularity is the greatest, both as a fanciers' and utility fowl. It has been truly said there is a time in the life of every fancier when he has a desire to breed the Light Brahma. There is a pleasing personality about the Light Brahma-stately and majestic and easy repose.

As an egg producer the breed holds an enviable record. This fowl produces a majority of its eggs in the cold months, when the product commands the highest market price. Many fail in egg production with these fowls because of a lack of knowledge as to how to properly feed. An overfed Brahma hen is an idler and is unproductve. As soft roasters no other breed or variety can equal the Brahma, nor are in such demand in the best markets of the country.

It can be stated as a fact that the latter day Cochanizing of the Light Brahma by many breeders has impaired the laying power of the breed where so bred. Mr. Felch and Mr. Williams and a number of other friends of the breed have strongly opposed this action, and have retained the Brahma in its true form. This Cochanizing of the breed has, too, affected it as an exhibition bird. The West will have none of the Cochanized stock.

\section{BREED CHARACTERISTICS.}

Standard weights of Light Brahmas: Cock, I 2 pounds; cockerel, ro pounds; hen, 9 I-2 pounds; pullet, 8 pounds.

Disqualifying weights: Cock not weighing nine pounds; hen not weighing seven and one-half pounds; cockerel not weighing seven and one-half pounds; pullet not weighing six pounds. 
In shape the head should be moderate in size. The pea comb should be small, setting firmly and evenly on the head, lower and narrower at front and rear than in the center. It has the appearance of three small single combs joined together at base and rear, the longest and highest in the middle, each evenly serrated. The serrations of the front and rear are smaller than those in the middle. "The breast should be full and prominent; the back short and broad. Neck well arched with hackle abundant in the male, flowing well over the shoulders. Body deep and well rounded. This is very important. Wings small and carried rather high. Tail medium in size; in male carried well upright. In female tail carried high enough to continue

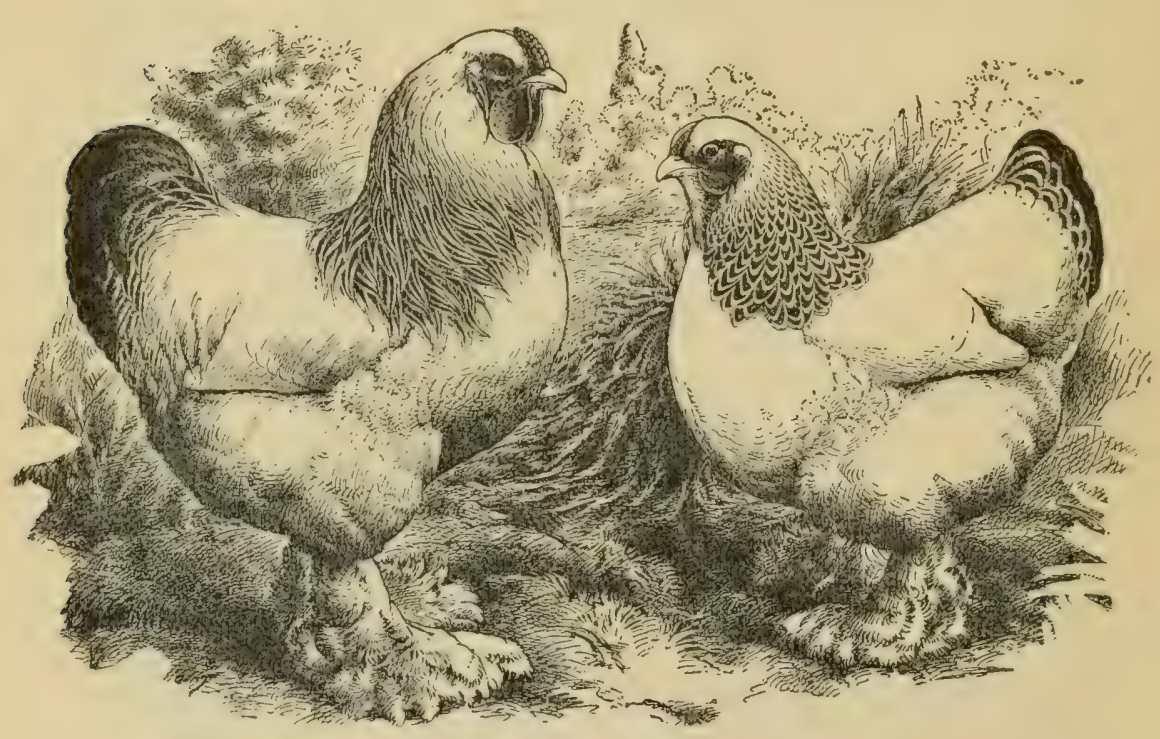

Standard Light Brahmas.

the concave sweep of the back. The thighs must be stout and covered with soft feathers. Toes well feathered on outside. In color the Light Brahma is pure white and black in the body, breast and thighs. Under the wings it may be white, bluish white or slate. Wings, neck and tail are positive black and white. Hackle web white with a black stripe down each feather, half or more its length, tapering to a point near the extremity. The wing bows are white, except front, where some black is allowed. Primaries, black or nearly so, with white edging on lower edge of lower web. In the secondaries the lower portion of the lower web is white, the rest being black. Tail black. Sickle feathers glossy greenish black. 
Standard weights of Dark Brahmas: Cocks, eleven pounds; hen, eight and one-half pounds; cockerel, nine pounds; pullet, seven pounds.

Disqualifying weights: Cocks not weighing nine pounds; hen not weighing seren pounds; cockerel, not weighing seven and one-half pounds; pullet not weighing five and one-half pounds.

\section{DARK BRAHMAS.}

The Dark Brahma should be identical in shape with the Light Brahma. In color the cock is silvery white, striped with black in upper part of body, including neck-hackle, back and saddle. Breast, pure black. Wing bows, silvery white. Primaries, black, except narrow edging of white on lower edge of

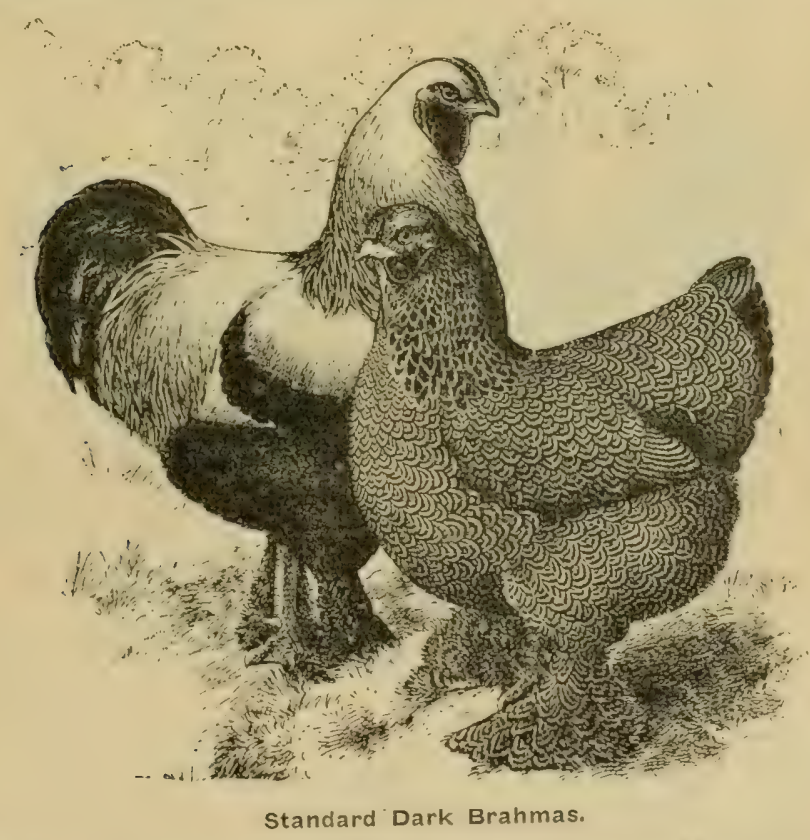

lower web. Secondaries, black, except lower half of lower web, which is white till near the end of feather. Here the white terminates abruptly, thus leaving the end of the feather black. Tail, glossy greenish black.

In color the female is a pure steel gray.

\section{BLACK LANGSHANS.}

The standard weight of all Langshans is: Cocks, 9 lbs.; cockerels, 8 lbs.; hens, 7 lbs.; pullets, 6 1bs. The disqualifying defects for Black Langshans are as follows: Specimens show- 
ing yellow skin; and yellow showing in bottom of feet; any but single combs; white in any part of plumage one-half inch or more in length, except in leg or toe feathering. Cocks that weigh less than $9 \mathrm{lbs}$; hens weighing less than $6 \mathrm{lbs}$.; cockerels weighing less than 7 lbs.; pullets weighing less than 5 lbs.; birds with shanks not feathered down the outer side; outer toes not feathered beyond the middle joint.

The head of both male and female should be of fair size, with a broad crown; beak well curved, dark horn in color; comb single, of good size, perfectly upright, evenly serrated with five points; neck well curved, with flowing hackle in male and well developed-plumage in female; back, flat at shoulders with a rise from center to tail, male with abundant saddle feathers; wing of medium size, well folded and carried up close to the

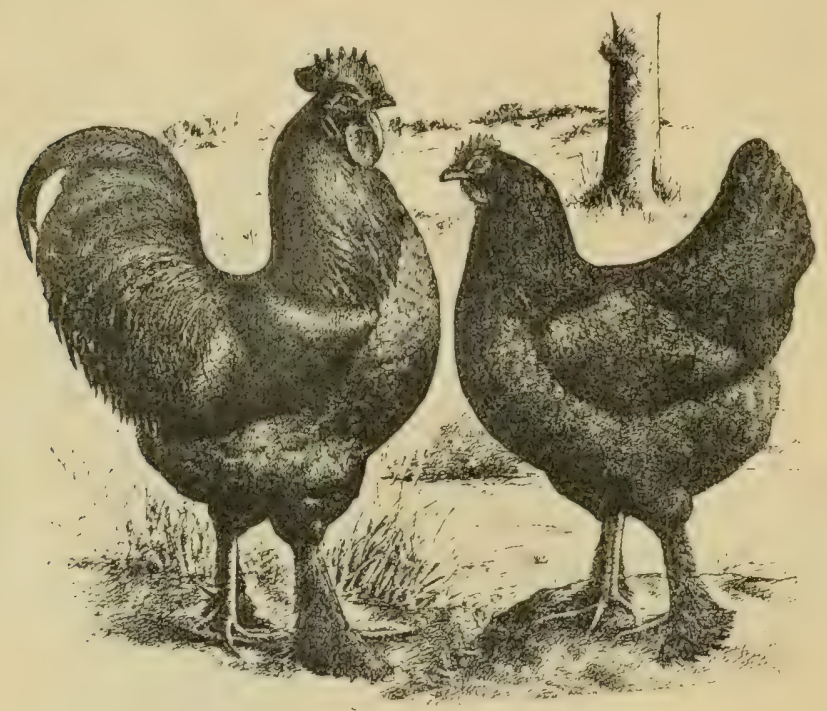

Black Langshans.

body; tail, large, full, carried well up, but not too close to a perpendicular line at the junction of the tail and back; sickles long, extendig well beyond the main tail; coverts, long, the longer the better. The color of both male and female should be glossy, metallic black, with greenish sheen. Purple bars and tinge a bad defect.

The Black Langshan has been rightly called the "Lordly Langshan"; no other standard fowl has the same graceful carriage, or well-bred showy appearance. From his broad crown and well-proportioned head points to his beautiful fountain tail, the Black Langshan male is a sight to fill the heart of any fancier with delight, while the hens are equally attractive and the prepossessing in appearance. 
Naturally these fowls have found great favor with the fanciers both in this country and in England.

Miss Coad, for many years one of the foremost fanciers and breeders of England, was the earliest importer and exploiter of Langshans, and to her able efforts I,angshans became known and bred all over the world. Miss Coad produced a strain of high station, fountain-tail Langshans that set the type for modern Langshan breeders, and came to be known as the "Coad" type, which is now recognized as the standard the world over.

It is an undisputed fact that the Black Langshan is one of the best layers of the larger breeds, and many contend that they will lay equal to the Am rican class, and some of the other smaller varieties. As table fowls they are extra in quality with the desired white skin, plump heavy bodies; in fact, they are ideal as a soft roaster.

\section{WHITE LANGSHANS.}

White Langshans are identical with the Blacks in everything but color which is pure white in all sections.

\section{THE FANCY BREEDS.}

Under this heading we shall consider those breeds that are bred mainly for their beauty, and while possessing many utility qualities are not bred to bring out these qualities but along lines to improve their appearance to the eye. In this class we have the Polish, of which the White Crested Black is the leader and the bantanns of which the Black Breasted Red Games, Silver Sebright and Buff Cochins are the most popular. All the Bantams are minature counterparts of the larger breeds irom which they take their name.

\section{WHITE CRESTED BLACK POLISH.}

The typical Polish must be of medium size with a large flowing crest, pure white in color, rising well in front so as not to obstruct the sight. The back should be of good length and the tail well spread. The comb is V shaped of small size, in fact, there is no comb at all the bird is preferred. The plumage is black with a greenish luster, free from purple bars.

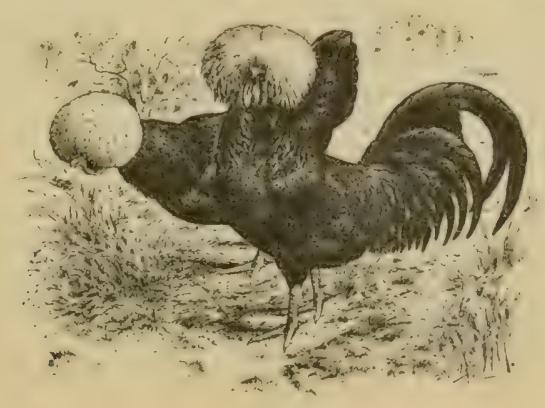




\section{Turkeys In California.}

VARIETIES.

Bronze Turkeys are the largest, hardiest, and by far the most popular of the varieties. An adult cock sometimes weighs over 50 pounds, though the Standard weights are much lower, i. e., adult cock 36 lbs. ; yearling cock 33 lbs.; cockerels 25 lbs.; hen 20 lbs.; pullet $16 \mathrm{lbs}$. The Bronze turkey is more popular than all other varieties combined, in most sections where turkey culture is extensively carried on.

White Holland Turkeys are second to the Bronze variety in popularity in many sections. They are much smaller, the Standard weights being: Cock 26 lbs.; cockerel I 6 lbs.; hen I6 lbs.; pullet Io lbs. Breeders of White Holland turkeys claim that they are much more docile and have less of the roving disposition of the Bronze, and are better adapted to close quarters or a narrow range.

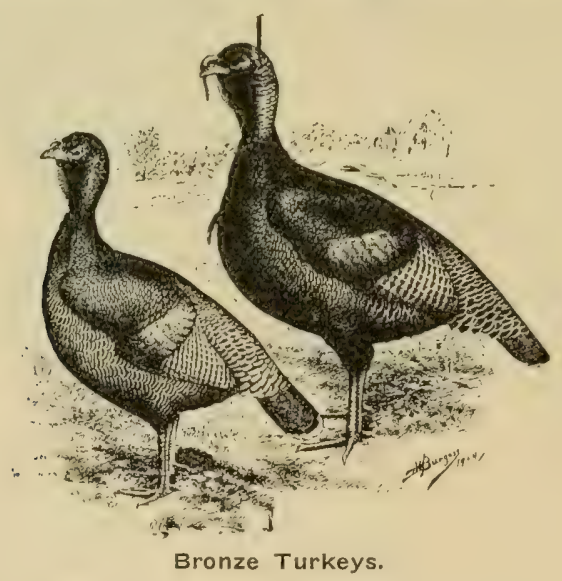

Narragansett Turkeys are often mentioned as the favorite variety with Rhode Island growers, but in a two days' trip through the best turkey growing section of that state with a friend a few years ago we found very few of them, both the Bronze and White Holland seeming more numerous. We did, however, see a few very fine specimens of the Narragansett. They are second in size, the Standard weights being: Cock $30 \mathrm{lbs}$.; cockerel $20 \mathrm{lbs}$. : hen I $8 \mathrm{lbs}$; ; pullet I2 lbs. In color they are gray, the plumage having a black ground with each feather tipped with a gray band edged with black. 
The other three varieties of the turkey are Buff, Slate and Black. All are quite rare in this country, and good colored specimens of the Buff and Slate varieties are very rare indeed. The weights for these three varieties are the same: Cock 27 lbs.; cockerel I8 lbs.; hen 18 lbs.; pullet I2 lbs.

But whatever the choice of breeds, it is more important that we consider the matter of breeding stock. The common practice of killing or selling off all the finest specimens at holiday time, keeping over only the late or stunted ones for next year's breeders, tends to deteriorate the quality of stock as well as the robust vitality that is one of the greatest requisites in successful turkey raising.

The earliest and thriftiest of the hen turkeys should be saved for breeders. These should be mated to two-year-old toms, giving each tom eight to ten hens. Gobblers are not really fully grown until $2 \frac{1}{2}$ or 3 years old, and are good breeders until 4 or 5 years old.

The breeding turkeys should not be fed on corn alone during the winter or their eggs in the spring will lack vitality. A good share of the ill luck in rearing young turkeys comes from neglect of the parent stock during the months when they are idle.

\section{THE CARE OF YOUNG TURKEYS.}

No food need be given the young turkeys the first day, but the mother should be fed. If the nest is so situated that a small pen can be made in front of it, it is a good plan to let the brood remain there quietly for some days. If the nest is not suitably located for this, the brood should be removed to a coop with small run attached. A coop with a board bottom is preferred, as the young turkeys are very susceptible to dampness, and a floored coop is dryer.

The pen for a brood of little turkeys should be made of boards 12 to $I 6$ inches wide, set on edge, and enclosing a space about. eight feet wide one way by twelve to sixteen feet the other. The little turkeys are at first much less active and rugged than little chickens, and should be confined to this pen for about ten days. It is better that, if possible, this pen should be on grass land, for little turkeys, like goslings, want green food from the start. If they cannot have a green run green food should be provided. Grit and charcoal must also be provided, and lice must be kept down by dusting with an insecticide the same as with young chickens.

Most authorities insist on the necessity of altogether avoiding dampness, keeping the young poults up while the grass is wet; but some of the most successful growers I have known let their turkeys range freely after they are strong enough, and say they observe no ill effects from such wettings as the chicks get. In- 
deed, their opinion is that this way of life is much better than the coddling methods.

While the young turkeys are confined to coops the ground under them and the coop itself should be kept clean. For keeping ground clean shifting the coop is all that is necessary if there is opportunity to do that. The coop must be kept dry, as well as clean. In wet weather when the floor becomes damp, it should be cleaned daily, and a liberal sprinkling of dry earth, or fine dry litter be applied.

By the time the young turkeys are two weeks old, if not before, the pen will no longer restrain them. Their roving instincts become plainly manifest, and in a very short time after they begin to go over the low sides of the pen they will go over fences four or five feet high with equal ease, and begin to make quite a circuit in foraging. Whether with hen or turkey mothers they require a good deal of watching at this age. In fact, while on a good range the turkey's pick a considerable part of their living, and need be little expense for food until fattening time arrives. They require more or less watching throughout the season, and an essential factor in successful turkey culture is to have some one keep an eye on the flock almost constantly to see that they do not wander too far, are not overtaken by violent storms, or picked off one by one by their natural enemies.

\section{FEEDING YOUNG TURIEEYS.}

We give herewith the method of feeding used by two of the leading turkey breeders of the country. Mr. Crangle gives only the feed used for the very young birds, while Mrs. Hargrave gives her method of fecding from the shell to the time of marketing :

\section{FOR YOUNG TURKEYS.}

After about 36 hours old, or after the hen leaves her nest, we feed for three or four meals equal parts of hard-boiled eggs and stale bread. After that mostly stale bread moistened with milk. For two or three weeks we give curdled milk to-drink. After two weeks we mix a little red pepper with the bread twice a week.-Crangle.

\section{FOR TURIEEYS FROM SHELI TO MARIRE'T.}

I feed poults every two hours until about ten days old, giving stale light bread softened in sweet milk (or water, squeezed dry, mixed with hard-boiled eggs, including shells finely broken. This food is alternated with bread and clabber cheese, oat flake and egg, or cheese seasoned with a little salt and pepper. After the little ones are about a week old I begin mixing a little whole or cracked wheat, Indian corn, Kaffir corn, or millet with the cooked food, and thus they learn to eat grain. Always try to 
feed no more than they will eat up clean each time. When they are about a week old I begin to drop the white bread, and give them instead what I call a brown light bread made the same as white bread, using one-half white flour (a cheap grade will do) and the other half about equal parts of shorts and bran, with a handful or two of cornmeal. The meal makes it crumble easily. The bread should be allowed to dry for a day or two before feeding; if fed fresh it may choke the poults. I gradually drop the white bread and eggs, and feed instead the brown bread and cheese. When about six weeks old they have become accustomed to the grain food, which, since they were three weeks old, has been kept by them in troughs in coops so constructed that the little turkeys can get in and the older fowls are kept out. By the time the poults are nine or ten weeks old I have dropped the soft or cooked feed to once or tivice per day. By September the older poults are dependent upon grain food and range. For fattening I had good results with a mixture of grains proportioned as follows: Two bushels whole corn, two bushels cracked corn, one bushel oats, one bushel Kaffir corn.-Mrs. Hargrave.

LICE.

Like young chicks, poults are troubled more or less with head lice and mites. The treatment for these are the same as with the chicks, which is fully covered in the chapter on lice and mites.

\section{ACCOMMODATIONS FOR TURKEYS.}

The turkey is essentially an outdoor fowl. Like the goose, it will generally by preference remain outdoors in all weathers, though that a part of this preference is due to habit would seem apparent from the fact that in continued very severe weather when they cannot feed outdoors, they scem to appreciate shelter and a comfortable spot to feed in. I kept a few turkeys in a room in my stable one season, letting them run about the barnyard and dooryard, but while the old birds seemed contented to keep within bounds, and were thrifty and bred well, they had to be driven into the shed nearly every night, and the young ones, after two weeks old were anywhere and everywhere but at home.

Turkey growers generally provide roosting places for their turkeys outdoors in a sheltercd place where the roosts can be high enough from the ground to protect them from marauding animals. The sheltered side of a barn or other outbuilding is a favored place for the turkey rnost. Occasionally a high open shed is used.

On most farms where turkeys are grown, the breeding stock is either not yarded at all, or yarded only during the breeling season, to prevent the hens straying away and hiding thei: nests. 
The hen turkey is very cumning in this matter, and I have known of their going a mile or more from home to lay, going and returning daily, and taking many precautions to throw anyone following off the track.

Some turkey growers yard their turkeys during the laying season. Some yard them until after the eggs have been laid each day, giving them liberty then, and of course seeing that they are back in the yard, and the gates closed, at nightfall. When turkeys are confined only during a part of the day, a yard $50 x i 00$ feet will do for a flock of twenty hens or less. The fence, if of wire netting, need not be over 5 feet high. A few turkeys might be kept permanently in an enclosure no larger than this, but when that is done higher fences are necessary, for immature fowls and hens that are not laying fly much more freely than the mature hens do during the laying period.

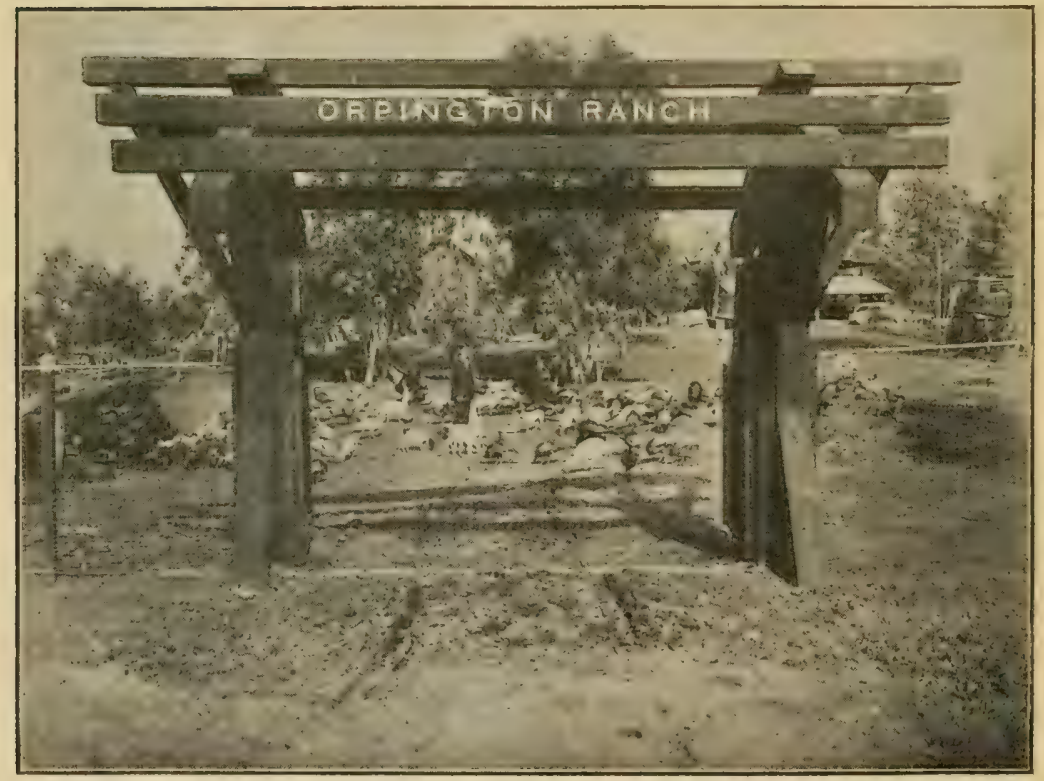




\section{Ducks In California.}

If we can judge by the number of inquiries received asking for information on duck raising, the interest in this branch of the poultry business is on the increase in this part of the world. We hope this is the case, for Southern California, and in fact the whole Pacific Coast, is perfectly adapted to this branch of the business, and the demand for good ducks is always far below the local production.

While there are fourteen different varieties of ducks described in the American Standard, breeders have settled down

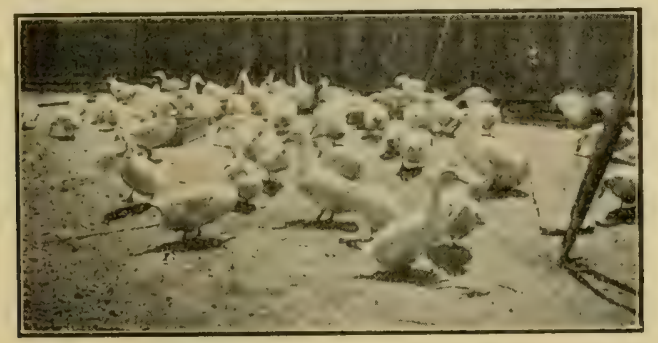

Pen of Young Californla Pekin Ducks.

to two varities-IVhite Pekin and Indian Runners-for profitable purposes. The Pelins are the recognized table duck, and the Indian Runners are the egg producers. In the matter of table ducks, the modern duck market calls for the production of "green" ducks, that is, of ducklings to be marketed at ten to twelve weeks of age. At that age they have frames almost as large as when full grown, and will dress four to six pounds each, five pounds being almost the average weight. The main profit in table ducks is undoubtedly in the green ducks, and the duck specialists devote themselves to it exclusively. Pekin ducks are much easier to handle in large numbers and in limited quarters than chickens. They grow so much faster that the brooding problem is greatly simplified, and if conditions are all favorable, and care anywhere near right, they are very free from disease.

The Indian Runners are marvelous egg producers, and have become great favorites with all who have bred them in California. It is safe to say that they will average 200 to 240 eggs for each hen per year, and this is below the reports given by many breeders who have kept records on them right here in Southern Cal- 
ifornia. The eggs from these ducks are hardly distinguishable from hen's eggs, are of a pleasant rich flavor with none of the strong taste found in other duck's eggs. Therefore Indian Runner eggs sell well in the market, and command as good, and sometimes better, prices than hens' eggs. It must not be understood that the Indian Runner is undesirable for table purposes, for they are excellent for this purpose, but do not make a good green duck, hence they are not sought after by the table duck men.

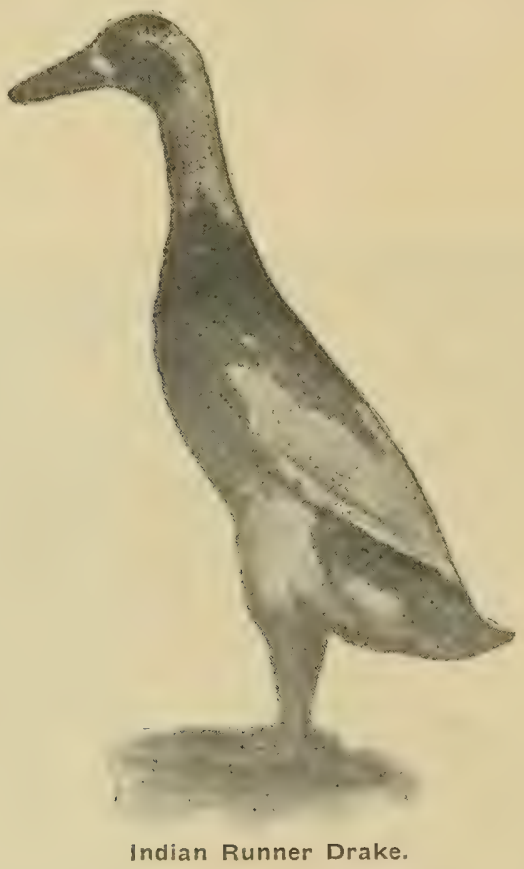

For growing the young ducks for market no water except for drinking purposes is needed. The ducks grow faster when kept from water. For the breeding stock, and for ducks grown for stock purposes, swimming water is not absolutely necessary, lut results are generally more satisfactory if the ducks can have access to a stream or the margin of a pond or lake. Contrary to the common idea, ducks neither require nor thrive in damp quarters. Though they like to frequent streams and marshy places, they need well-drained ground to which they can go when tired of the water, and the house site should be on well-drained and if possible dry ground.

Houses for ducks are built on the same general plans as those used for hen houses. Houses can be made of the cheapest material, and it is not necessary that they should be warm, but 
they should be as dry as possible, hence a good roof is desirable. A house used by many of the large duck raisers is 16 feet wide, 20 feet long and 6 feet on the side and 8 to 9 feet in the middle. Through this house runs a passage way two and a half feet wide, and the house is divided off so as to accommodate a number of pens at one time. The fences for yards should be of wire. A fence two feet high will keep ducks in. A good plan is to make temporary fences, driving short stakes into the ground and attaching the wire fencing to these with staples, using only two or three at each stake, and not driving them in tight. Ducks foul the ground very badly: and it is desirable to move them fre-

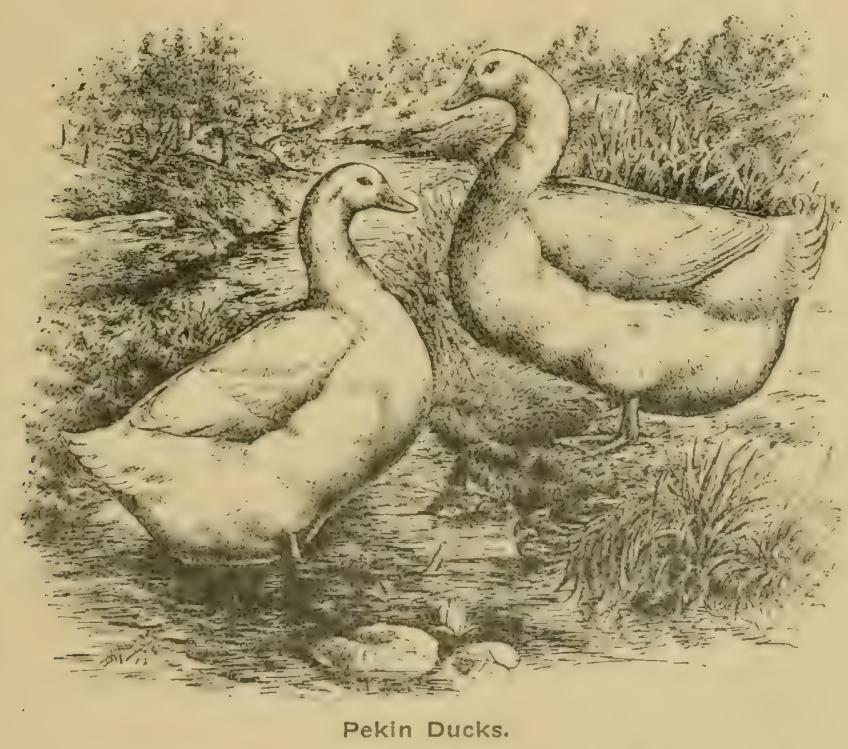

quently and turn over and plant the ground they have been running on. As drakes are not as combative as cocks are, flocks large enough to require a number of drakes are generally kept together. Twenty-five or thirty ducks can be kept in a flock. For this number of ducks five or six drakes are needed.

Successful breeders have found that ducks do best on soft food, and most of the largest breeders are feeding both their young and old birds on soft food exclusively. WVe give below the rations fed the breeding stock by four of the leading duck breeders in the United States:

I. "Equal parts of cornmeal, wheat bran and low-grade flour, with about twelve or fifteen per cent. of animal food. One fourth of this food should be composed of vegetables cookedsay, small potatoes, turnips, etc., with all the green rye and refuse cabbage they will eat. Feed this mixture mornings and 
evenings, giving a little corn, wheat and oats at noon. Feed all the birds will eat up clean and no more."-James Rankin.

2. "Equal parts cornmeal and shorts with ten per cent. beef scraps added. If green food is not available, add one-fifth cooked vegetables to the mash. Give raw vegetables at noon two or three times a week."-Weber Bros.

3. "One-fourth cornmeal, one-fourth bran, one-eighth broken crackers, one-eighth gluten meal, one-eighth low-grade flour, one-eighth beef scraps. This mixture makes two-thirds of the mash, the other third being scalded green clover cut fine, and boiled potatoes mashed. The grain feed is mixed dry at first, and then the clover and potatoes added, and the whole mixed with hot water and fed warm. Feed all they will eat up clean with a relish. Allow no food to stand before them at feeding times."-Curtiss Bros.

4. "Two parts bran, one part Indian meal, two parts cut clover or other green food. Ten to twelve per cent. of the whole to be best quality of beef scraps."--Pollard.

5. "Two parts clover heads, boiled, two parts cornmeal, two parts middlings, two parts bran, one part ground bone."$\mathrm{McF}$ etridge.

Where a breeder wants to hatch only a limited number of ducklings, hens can be used to good advantage, but where a large number are to be raised, incubators and brooders are indispensable. When eggs are hatched under hens, give each hen nine to eleven eggs, according to the size of the hen and the eggs. Duck eggs vary in size, and some will allow the setting of nine, while others do not overcrowd the nest when eleven are given to the broody hen.

When only a few ducklings are hatched at a time, hens are satisfactory as mothers, but if the number is great enough to use a brooder, it will be found a much more satisfactory method of raising them. The same general rules for running a brooder for chicks apply to ducklings. We give below rations for ducklings used and recommended by the greatest of all modern duck raisers, Mr. James Rankin:

"First week-Equal parts of cornmeal, middlings, crackers or stale bread, and green stuff; mix in a small handful of sand to each quart of food. Give occasionally bread soaked with milk for a change.

"Second week-Four parts cornmeal, two parts wheat bran, two parts middlings, one part beef scrap, sand; mix with about one-third the quantity of green stuff. At about six weeks put ducks in fattening pens and feed two-thirds meal, the remainder about equal parts of bran, middlings and greens; add about twelve per cent. of the whole beef scraps."-Hallock.

"First four days-Four parts wheat bran, one part corn- 
meal, one part low-grade flour, five per cent. fine grit. Feed four times a day what they will eat up clean.

"From four days to three weeks-Three parts wheat bran, one part cornmeal, one part low-grade flour, three per cent. fine grit, five per cent. beef scrap, soaked. Finely cut green clover, rye or cabbage. Feed four times a day.

"From three to six weeks old-Equal parts cornmeal, wheat bran and low-grade flour, ten per cent. beef scrap, three per cent. grit. Feed three times a day.

"Eight to ten weeks old-One-half cornmeal, equal parts bran and low-grade flour, ten per cent. beef scrap, three per cent. grit; oyster shells and less green food. Feed three times a day.

"The above ingredients should be made into a mash, and should be crumbly, not pasty. Proportions by measure, not by weight."

Both old and young ducks need water constantly before them, and especially at feeding time, as they must wash all their food down with water. Ducks drink even at night. It is best to use a drinking fountain that the ducks cannot get into and foul the water. Give a pen of ducks plenty of water fountains so that they will not crowd around one fountain; especially is this bad with young ducklings, as they frequently injure one another in this way.

The Standard weights for Pekin ducks are: Adult drake, 8 pounds; young drake, 7 pounds; adult duck, 7 pounds; young duck, 6 pounds.

Standard weight of Indian Runner ducks: Drakes, $4 \frac{\mathrm{T}}{2}$ pounds; ducks, 4 pounds.

Standard disqualification of Pekins: Bill or beak of drake marked with black; feathers showing any color other than white or creamy white; twisted wings; crooked backs; awry tails.

Standard disqualifications of Indian Runners: Claretcolored breast; blue wing bars; twisted wings; crooked back; awry tails. 


\section{The Goose In California.}

Interest in goose raising is at present very slight in this state and the demand is considerably above the supply.

No doubt interest in goose culture on a commercial scale could develop considerably before an over supply was created, but those to whom goose growing should appeal most at present are the many who might grow a few geese every year, either for home use or for a local market, almost withoit expense. Almost every farm has some land not used for any other purpose and not productive, that might be devoted to goose growing. On almost every farm a flock of geese, large or small, according to accommodations, might be kept on pasture with other stock.

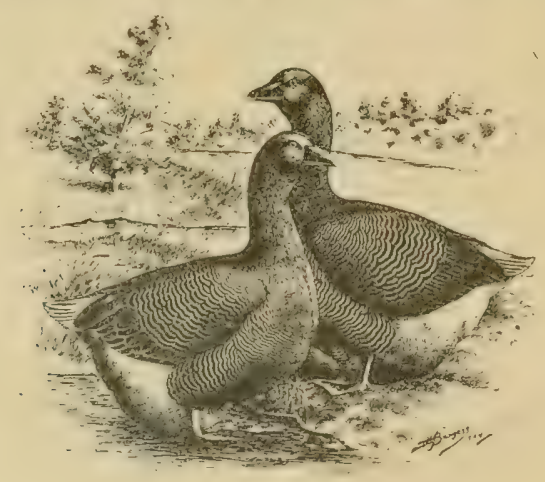

Geese thrive most readily and are least trouble to handle, and grown at least cost, when given good pasture, but they may be grown like ducks in close quarters if green food is liberally provided. This way of growing them is not recommended. I merely mention it as possible for those who would like to know snmething of geese, but have not good natural facilities for growing them.

Under favorable conditions geese are the easiest of all domestic fowls to handle. With good pasture provided with water they may be left to themselves half or more of each ycar. They do better, generally, if provided a little grain food to supplement what they get by foraging, but it is not regarded necessarv, and perhaps the majority of those growing geese give the old stock nothing during the pasture season, and feed the goslings only a few weeks. 
THE BREEDS OF GEESE.

Many geese found in this country are the common gray, gray and white, or white geese, not much larger than a large duck, and in a general way showing the same inferiority to the improved breeds of geese that common fowls of all kinds do to the well bred.

Of pure bred domestic gecse there are the following breeds: Toulouse, Embden, African, and China, or Chinese. Of the last named breed there are two varieties: Brown and White.

\section{GETTING A START WITH GEESE.}

If one wishes to grow more than a few goslings year after year the best plan is to begin with adult birds three years old or over. Geese do not come to full maturity until about three years of age, and rarely give at all satisfactory results until two years old. The young geese will lay and some of the eggs may hatch, but the goslings are apt to be weakly and not thrifty.

Good stock of any of the improved breeds usually costs $\$ 3$ to $\$ 5$ per bird. It is better to purchase sume months in advance of the breeding season, as the geese will not breed well if moved just prior to the laying period. If stock has not been purchased early it is usually better either to let the matter go over for another season, or to buy eggs. In any case one must expect it to take several seasons to get a flock of geese established in new quarters, and breeding satisfactorily, for good breeders of mature age are not often offered for sale, and the young geese require two years, and sometimes more, before they produce well. Once established, however, a flock of breeding geese can be kept unchanged for quite a long period. The females are said to be profitable up to ten or twelve years of age, and males to a little more than half those ages, say, six or seven years.

From one to four females are mated with each male. Young geese are disposed to pair. As the ganders grow older they will usually serve more mates, but they do not copulate indiscriminately as fowls and ducks do. Each gander serves only his own mates, and an unmated goose in a flock in which all the ganders have other mates may be entirely neglected. For this reason goose growers often put an extra gander with the flock to pick up and mate with any neglected or discarded females. Usually when a gander and goose have mated once they are faithful to each other as long as allowed to remain together, and often refuse to take another mate for a long time after being separated. This trait makes it possible once the geese are mated to allow as many families as desirable to run in one flock, even though they be of different breeds. 
HOUSES AND FENCES.

Geese require very little shelter, and the heavier breeds are restrained by almost any sort of low barrier.

A low rough shed open to the south will provide all the shelter needed, and it need not be much larger than will give the flock standing room under it. Sometimes they have to be confined at night for protection, but, as a rule, they can take care of themselves.

\section{CARE OF BREEDING GEESE.}

Geese breed best on pasture with streams or ponds accessible. They require little grain if the pasture is abundant. If pasture is short it may be supplemented by grain and vegetables. In summer they should be fed mostly on vegetable food, cut clover, alfalfa, cabbage, beets, turnips, etc., with oats or barley and bran. Corn is generally too fattening, and most breeders are very sparing in feeding it. Herewith are given the rations of two well known breeders of geese:-

r.- "Through the laying and breeding season, in addition to grass, they should be fed twice a day with shorts and corn meal, equal parts, thoroughly moistened with cold water, but not too wet lest it produce diarrhoea; add ten per cent of beef scraps, or its equivalent." Rudi.

2.- "Take some boxes about eight inches deep, and put oats or barley in them. Place these in the pasture away from other fowls. There is no danger of overfeeding grain if the geese have pasture. Give corn only in coldest weather."-NEWMAN.

Geese usually begin laying in the winter, or in the early spring. When the goose is ready to lay she is apt to seek out a nest for herself, and having once selected a place is impatient of any interference with her laying habit. The goose, as a rule, looks for a somewhat secluded place, and as a pasture often furnishes few such places, keepers make a practice of providing nests that may attract the geese. An empty barrel placed on its side with a little earth in it to hold it steady and make a foundation for the nest, then a little straw or hay added, is the usual nesting place furnished. Often the geese will ignore these and make their own nests right in the open.

As geese lay but few eggs it is customary to take them away as laid during the first laying period, and give them to hens to hatch, or place them in an incubator, and not allow the geese to incubate until they have laid a second lot of eggs.

\section{HATCHING GOSLINGS.}

The hatching of goslings by natural methods, whether with hens or geese, differs little from the hatching of other fowls. 
The points of difference in hatching with hens are those which depend upon the size of the eggs and the length of the period of incubation. The eggs of the large breeds of geese are very large, and early in the season three will be fuund enough for a medium sized hen, Later when the atmosphere and ground are warmer more eggs may be given, but it is rarely wise to give more than five large goose eggs to a hen. The period of incubation being thirty days, approximately half as long again as the incubation period of hens' eggs, hens that are not in good condition and free from lice are likely to neglect or desert their eggs. Hence besides taking special care to use hens that are in good condition and to guard against lice, which multiply much faster on a hen late in a prolonged period of incubation than while she is active and robust, it is well to watch the hens very closely after the third week of incubation, and remove any that seem to be losing condition, giving their eggs to fresher hens.

When geese are used for hatching they may he allowed twelve to fifteen eggs. Usually they must be set where they have been laying. Niany growers simply leave the eggs of the second litter in the nest.

Goose eggs can be tested from the fourth to sixth day, and the infertiles romoved. Testing should always be attended to with the early hatches and under hens. With eggs set under geese late in the season it is not so essential, for the eggs usually run very fertile then, and as the incubating goose does not like interference it is as well to let her alone.

FOOD AND CARE OF GOSLINGS.

The first two or three days keep them in a warm place and give a little soaked bread and water. In nice weather turn them out in small enclosures which can be moved every day. After a week let them go. The first four or five weeks give nothing but stale bread occasionally, but always leave them at liberty to get all the grass or clover they want. Do not soak the bread, as they do not like it so well. After five weeks give a mash of two-thirds bran and one-third corn meal. To fatten-after six weeks, feed one-half bran, one-half corn meal; do not feed it sloppy. Never allow goslings to go to the water until fully feathered, and then only let those go which are to be kept for breeders. 


\section{Pigeons For Profit.}

\section{A BACKYARD SQUAB PLANT.}

So many of the housewives are looking for something out of the ordinary for Sunday dinners, the little luncheon in honor of the stranger within the gates, and other special occasions, that a flock of fine squab breeders is becoming almost a necessity to a family. There is nothing more delicious than a tender, fat squab, smoking hot, with a crisp piece of bacon broiled with it, and by giving the birds the proper care there is a sweetness and flavor to their flesh that no market bird will have. Another feature there is always a demand for good squabs at a high price for the table, or by the pair for squab breeders. With squabs, as with poultry, the common variety pays the smaller profit, the markets paying $\$ 1.75$ a dozen in the summer and $\$ 3.00$ in the winter for the common squabs, which are generally dark and scrawny, while a thoroughbred squab will be light, fat and better flavored. Another argument in favor of good stock is they breed much oftener than do the common ones and the markets will pay much higher prices for them. There are any number of good squab breeders, the working Homer being the leading favorite. They are a very prolific pigeon, breeding from six to nine pairs a year. Fine large birds of this variety will give entire satisfaction. They also do well crossed with the Hen, Runt or Dragoon. The Dragoon is the next best bird and almost equal to the Homer in breeding and feeding qualities. It is a fine show bird and I think will be a great favorite. This is also good crossed on Homers, Hens or Runts. The Runt is a very large pigeon, the largest of all breeds in fact, but as a squab breeder not a practical bird at all if used pure. They are not a prolific bird and poor nurses, but crossed with a Homer or Dragoon give size to the squabs and take prolificness and good feeding of the young from the smaller bird. Of course in their pure state there is a good profit in selling them for breeding, for a good Runt will always command $\$ 8$ to $\$ 15$ a pair. The Hen breeds oftener than the Runt but not as well as the Homer and Dragoon. It is a large pigeon and a good feeder of its young. They are frequently crossed by squab breeders. The Hen also commands a big price. They are also known as the Florentine Runts.

Now for the loft. You know, of course, that's what a pigeon's house is called. You have a back fence seven feet high. Lay tar paper carefully over a space eight feet wide and 
the height of the fence. Bring out a partition three or four feet on each side of the eight foot space just to make it more private. Nail heavy cleats on the partition boards and place a shelf every twelve inches. On this shelf place your little nest boxes, a box for every pigeon, with one landing board to the two boxes. A male bird will try to take up a whole section so make two of the nest boxes rather private by having the landing board between and he will be content to have only two of them. I do not consider nappies a necessity, but they certainly do look rather pretty. A foot from the top of the fence build a sixteen foot roof of lumber covered with roofing paper. At the very top stretch a wire covering for the entire top, making the pen 30 to 60 feet in length; wire in the sides also, with a gate at the far end. The covered roof is always a favorite place with the pigeons for sunning themselves and in the flight a number of perches should be placed. This makes a fine pen for twenty-five pairs of breeders:

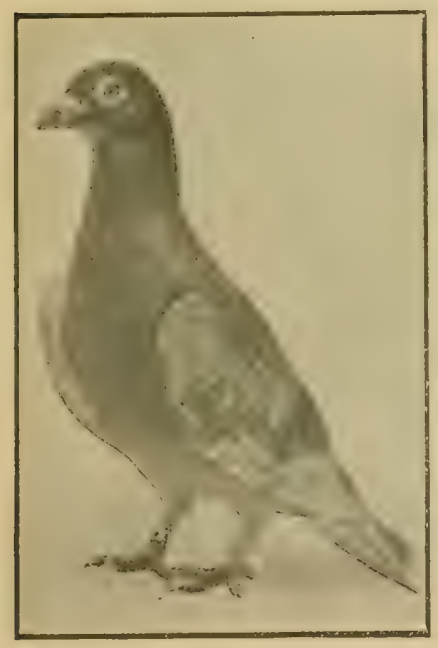

Squab Breeding Homer.

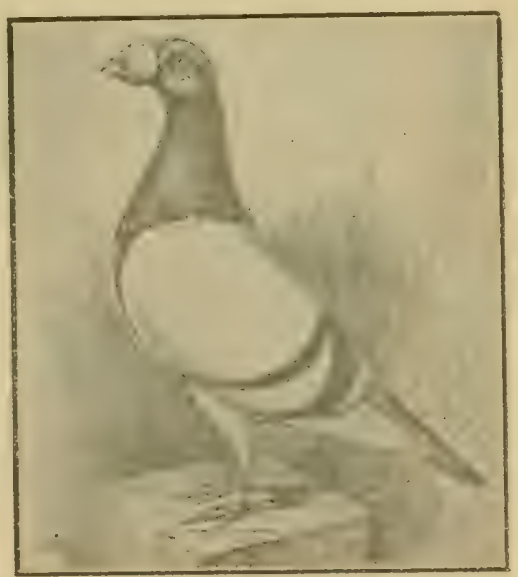

Blue Barred Squab Homer.

Pigeon grit should always be before them, and hard salt. A very good way to feed salt is to get it in ten-pound bags, pour sufficient water over it to wet it thoroughly and put it in the oven and bake hard. It will be hard but softer than the salt purchased for the purpose at the feed store. Pigeons to do their best must have an abundance of pure water, both for bathing and drinking. Now as to food. Good sound wheat is the staple, I find, with corn, good, dry cracked corn, a close second. No poor food is cheap. Canada peas are much relished by the birds, but as they are fattening should be fed sparingly during the breeding season. During the moult, hemp, 
peas and vetches are good. Give a bit of green food occasionally. Stale bread is also enjoyed by the dainty little creatures and a little dessert once in a very great while of a hemp and anise seed will make every bird come flying when they see you. Many people like the idea of hopper feeding, leaving everything for their use constantly before them in hoppers built so the food cannot be wasted, while a like number fancy the birds do better if fed regularly twice a day. Now your birds have a comfortable house, plenty of clean water, grit, shell, salt, nesting material and will soon go to housekeeping.

In this business too much cannot be done to make the breeding birds comfortable and to keep them in health. Many leave their birds go neglected and soon have nothing but lofts of sickly

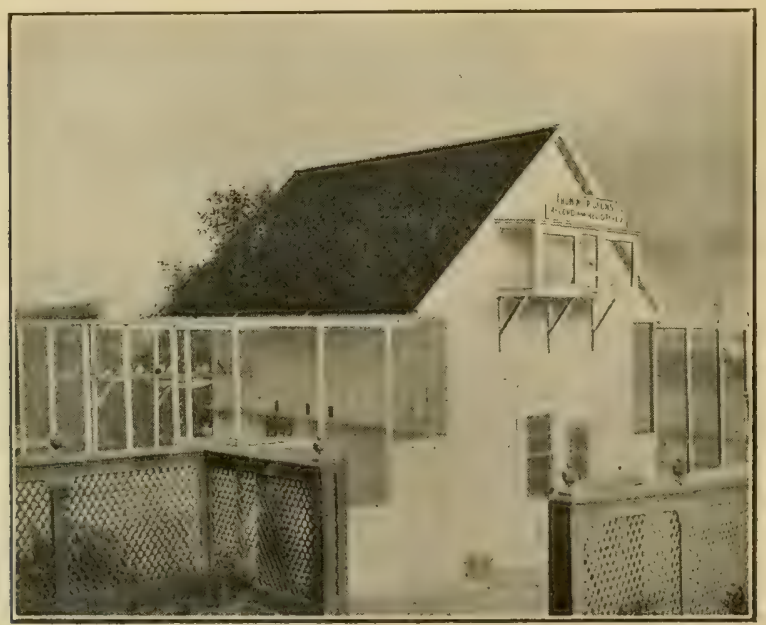

Sucessful Callfornia Squab Plant.

and unprofitable birds and finally they have nothing to speak of except the lofts themselves. If one goes into squab raising there is enough money to be made in that alone. When one finds only one squab in a nest, and also another nest with only one in, about the same age, place both together in one nest. This relieves one pair, which will commence building at once and rear another pair. This method gains time, for while each pair have but one, they can rear two just as easy, ot."erwise there are two pair raising one pair of squabs. No fear need be entertained that a pair of birds will not feed another's young; they differ in this respect to all the wild birds.

Extra pains must be paid during the moulting period, which begins in the middle of August and lasts a month or more. It is during this period that pigeons become sickly and many often 
die, even with the best of care They are weakened by this throwing off and growing feathers and need to be treated accordingly, i. e., given food to strengthen and stimulate, so as to hurry them through their moult and become strong again; they are off their feed as a rule and appear lazy Don't imagine, as some do, that their birds are all going to die, for if given proper attention they will pull through all O. K. Remember this is the most trying season on pigeons and the best food should be given. A rusty nail in their drinking water is good at this time. Do not expect many squabs during the moult, for birds cannot moult and breed too.

For the nest material use some good strong tobacco stems with their other nesting material. It is best to cut them about six inches in length. The vapor or aroma which arises from these stems keeps away lice upon the squabs and also on the old ones to a certain extent. We never use lice killer or anything else as tobacco stems answers all the purpose. Stems should be obtained direct from the cigar factories. Allow the birds to use all they wish winter and summer, as they are cheap.

To prevent lice, besides the stems being used, the pens and nests must be cleaned occasionally. As soon as the squabs are taken out of the nest, rcmove the filthy nest for they are what create mites, worms, etc., and make cleaning miserable work if the nests remain too long.

There is no use in pigeons sitting upon infertile eggs, for they lose in time, and add expense for feeding, deriving no benefit therefrom. After eggs are set upon for five or six days take them out of the nest and gently hold them to the light-sunlight is best-infertile eggs will be almost transparent, while eggs that will hatch have on one side a number of veins all running together towards the center, the whole resembling a spider. If both eggs do not have this spider appearance in at least seven days they will not hatch, and there is no use leaving the birds sit on them; destroy them and in a few days new eggs will be laid.

A word here as to the cause of infertile eggs may be of interest to the reader. First of all it may be the fault of the hen. Feeding highly stimulating food oftens fattens to such an extent as to cause infertile eggs. This will often cause soft shelled eggs, although in the majority the latter is caused from insufficient supply of egg shell material; lime or oyster shells will remedy this. The cocks are most frequently the cause of infertile eggs, due to their loss of stamina. A good reliable way to remedy this in the cock is to clip off fully one-half his tail feathers. This works like a charm, although we have been unable to discern its cause. We have had pairs, always having infertile eggs, cured by this method. Sometimes pulling out the entire tail will answer. This is likewise very good for weak moping pigeons, and it is an excellent thing to do with young birds when 
they enter their first moult. Many die at this period, and this often saves a great number.

Both the cock and hen pigeon take part in sitting upon the eggs; the hen sits from about 4 o'clock in the afternoon till about $100^{\prime}$ clock in the morning; the cock relieving her the rest of the time. Two eggs are laid one day apart. Eighteen days sitting is required to hatch the eggs. The first egg hatched is the cock, and it is from this that the cock as a rule is the larger of the two, as he is shown partiality and fed more. When the egg is about to hatch, a secretion, known as pigeon milk is formed in the parent birds' throat, and this is pumped into the squab's mouths and fed them until they attain an age of about ten days, when grains are fed after being predigested. After they are two or three weeks of age the parents feed them upon the grains soon after they have eaten them and drank water. They take the bills of their young into their mouths and by a sort of pumping motion draw up the food from their own crops and feed it to their young. Pigeons also have the power to dislodge any foreign substance which becomes lodged in their crops, such as oyster shells, etc.

The squabs to be of marketable size should be about four weeks of age, although some parent birds by good attention and frequent feeding have their young well filled out and fattened in less than four weeks time, others again require a longer time; however, when the pin feathers under the wings and upon the sides of the body are a quarter to a half inch in length, the squab is fit to kill, as it will not improve but rather decline if left to get older. Nany in starting this business kill their squabs entirely too young, but all live to learn and it does not take long in the squab business. The squabs continue growing and fattening until they begin to fly, and one day of this exercise reduces their weight considerably. It is a good idea to cut one wing of the squab to prevent flying, when ripe instead of green market squabs are wanted. While experience is the best teacher, beginners should profit by others' experience, which can be gained by visiting. successful California lofts.

\section{SELECTION OF STOCK.}

The foundation of most successful squab ranches are purebred Homers, The pure Homer are the best breeders, and one finds a greater percentage of fertile eggs and a smaller percentage of delicate or sick youngsters among their offspring, and less sickness or tendency to slack up in breeding, among them than any other variety. Good Homer squabs should run from 9 to I 2 pounds per dozen; 12 pounds to the dozen is the ideal squab. but to obtain this breeders must cull their birds many times. It can be done with pure Homers, if only the largest breeders are selected each year. Extra large Homers are not easy to get at all times, as the hens will run small under the best conditions, 
and quite often a nice large cock mated to a small hen will breed extra large young ones. The hens as a rule are more tight or close feathered than the cocks, and are much heavier than they really appear to be. It is not safe to cull out the small ones too close or one is likely to have a heavy surplus of cocks.

A thoroughly mated pair of Homers will instinctively begin hunting a nest or corner they can call their own, as soon as turned loose in a new loft. For this reason it is well to have the loft ready for the birds before they are put in it. The nest boxes should be all arranged, nest pans in place, feed and water dishes, etc. in working order. If mated birds have been purchased the best method is to liberate as many pairs as you intend breeding in a loft, all at the same time. When this is done, each pair have the same opportunity to select their nest, and they will settle very readily.

If part are placed in the loft ahead of the others, the first occupants have the advantage over the new arrivals, of being acquainted with the place and the result will be considerable fighting before all are settled.

The mated-pairs will all start looking for a nest and one by one, settle in what is to be their future headquarters.

Once a pair settles on a nesting place, mark the nest and mark the pair. In this way one can easily keep a record of just how many young one gets from each pair of breeders, the date they laid, etc. The birds all settled one does not have long to wait for the first eggs.

As stated, the Spring months are the best for breeding. We do not believe in breeding from a pair the year through and strongly advise separating the old breeders for a month or two during the summer, in order that the hens may rest and get ready for another season's work. Our plan is to start the old brecders about February ist, and save all the strong youngsters hatched during the months up to July ist. These youngsters will all be ready to start breeding by the last of September, and can be set at work as fast as they begin to show an inclination to pair up.

These young pairs are the ones to get the winter Squabs from. They will breed well all through the winter, and the following season up to moulting time. We would advise marketing all squabs hatched after July ist. These as a rule will not breed until late in the winter or the following spring.

\section{HANDLING OF PIGEONS.}

One of the things a beginner in the breeding of pigeons needs to learn is how to handle the birds properly. Nothing will show one's experience with pigeons quicker than the manner of handling them. A practical fancier holds the bird in such a way that he cannot struggle, and in such a way that its plumage is never ruffled or injured. For the benefit of the novice we give a few instructions. 
When possible to do so, catch the pigeon between the two hands; (we have seen breeders so expert at this that they could catch a bird on the fly and get him right), or by clasping the hands over the back, so as to cover the wings and keep them folded. Once caught, no matter how, turn the right hand so that the back shall be to the right, thumb uppermost; then place the legs of the bird between the first two fingers, close down the the palm of the hand, bringing the two fingers close together thus securing the legs, then curve the thumb over the wing flights and tail so that it touches the end of the first finger; then turn the hand downwards, bringing the remaining fingers up towards the body. You then have the pigeon so that he can be readily examined, cannot struggle, and no matter how wild, he is under perfect control.

By carefully following these instructions a little practice will enable any one who has occasion to handle pigeons to do so correctly, with pleasure to himself and comfort to the bird.

\section{DISEASES OF PIGEONS.}

The following on diseases of pigeons was written by Dr. C. M. Selfridge, Oakland, Cal., and has proven of much value to California breeders of pigeons.

\section{A POPLEXY.}

The symptoms-The bird turns its head down with the top resting on the battom of the cage. Inability to eat; incordination of the leg muscles; staggering.

On post mortem examination you may find a ruptured blood vessel, and if of long standing, softening of the substances of the brain.

Causes-Overfeeding of too rich, fattening food; lack of sufficient exercise; inbreeding.

Treatment-Usually the axe. One of our members succeeded in curing two cases in the following manner: Epsom salts, three two-grain doses, followed by one-grain dose of quinine, not to exceed three grains in forty-eight hours.

\section{BARRENNESS.}

Symptoms-Where the hen keeps her nest for days without laying. The mate then takes his turn, setting on the empty nest.

Causes-Inbreeding, which weakens the reproductive organs in both sexes. Too rapid and frequent laying one season will cause a hen to be barren the next season, or she may lay very late.

Treatment-Putting a pair of fertile eggs under the birds will frequently cause them to go to breeding. Strychnine given in $I-400$ of a grain twice a day may cure the trouble.

Feeding boiled meat, slightly salted, has proven a very valuable remedy where all others have failed. 
Darling's Meat Scraps, fed dry, will not only correct this trouble, but will increase the frequency of nesting and mature the squabs sooner. Should all of these methods fail, you can use the birds for nurses, to hatch fertile eggs laid by another pair having youngsters that would be too young to care for themselves when said eggs are due to hatch. If you do not care to do this, put them in the pot.

\section{CANKER.}

Symptoms-Resembles diphtheria in the human. You will find patches of a white substance on the mucus membrane of the mouth and throat. On removal the wound bleeds quite profusely, sometimes, so it is not a good plan to remove the canker forciblv.

Causes-Inbreeding; bad food; impure water; cold.

Treatment-Apply with a brush, equal parts of Hamlin's Wizard Oil and sweet oil twice a day. Also, dilute alcohol 20 per cent, applied with an ox-hair brush. Give Federation Specific No. 4.

\section{CISTIC FORMATION}

Symptoms.-A collection of a cheesy mass at any of the orifices of the body, or in places caused by injury.

Causes.-In some cases it is due to an injury. They are found in the egg passages in young hens after the passing of the first egg. In this instance, remove it by general pressure, about the vent with the thumb and finger, using the round end of a hair-pin to lift it from the passage.

In the treatment of these growths, it is best to remove them and dust in a small quantity of Iodoform; sometimes an application of 50 per cent carbolic acid and sweet-oil once a day will prove efficacious.

\section{COLD}

Symptoms.-Sneezing; running of a thin, watery substance from the nostrils; breathing through the mouth; with a collection of jelly in "V" or roof of the mouth.

Causes.-Change of temperature; lowering of vitality by not eating, especially in youngsters.

Treatment.- The removal of the jelly with an ox-hair brush, dipped in Hamlin's Wizard Oil and sweet-oil. Apply also to the nostrils. Give Federation Specific No. 5.

CROP BOUND-SOUR CROP.

Symptoms.-Bird refuses to eat; food does not pass from the crop; bird throws up food, drinks to over-distension; water runs out of the mouth as the bird is picked up.

Causes in Poultry.-Where the chicken eats large pieces of meat, or some large substance that will not pass into the gizzard.

In pigeons, where food does not pass readily. Impure water or food may cause it. 
Non-hatching of the eggs, which causes souring of the milk in the crop..

Treatment.-Removal of all food from the crop, washing out with bicarbonate of soda water; restrict diet; give hemp seed; small quantities of water; give Federation Specific No. 3 .

Occasionally Federation Pill No. I.

\section{DIARRHOEA.}

Symptoms.-Water discharge; rapid emaciation; often time with good appetite; frequent thirst.

Causes.-Cold; musty food; impure water.

Treatment.-Change of food and water. Give Federation Specific No. 2.

\section{DROOPY WINGS.}

Where the wings drop down and drag on the ground; caused by picking the bird up by its wings; lack of muscular development, especially in growing squabs; sometimes.you may find lice as a cause.

Treatment.-Cutting the long flight feathers, which lessens the weight of the wing, and the weaker muscles will draw the wing up. the head.

For the lice, dust with any of the lice powders, also grease FEATHER DISEASE.

Where the feathers do not come through the skin, but spread out underneath. Quills do not drop the capsules.

Causes.-Lack of proper feather forming material.

Treatment.-Grease well with vaseline; put lime in the water. Federation Pill No. 2.

\section{GOING LIGHT.}

Bird becomes very much emaciated; refuses to eat or drink; dumpy; ruffling up all its feathers.

Causes.-Repeated laying and not nesting; confinement; starvation.

Treatment.-Plucking the tail, which causes an irritation, and sets them eating. It is better probably to squeeze the oil duct at the root of the tail. The Pigeon Pills No. 6 will often prove beneficial. Also Federation Pill No.2. One grain of Epsom salts at night, with one-half grain of quinine in the morning, has in some cases proved beneficial.

Putting the Whitman Tonic in water has saved a great many good birds.

Formula:

Creosote, 20 drops;

Ferum Sulph., 2 drachms;

Magnesia Sulph., 2 ounces.

Sodium Sulph., 4 ounces.

The use of dried ingredients in compounding the same will avoid the remedy from solidifying. Use teaspoonful in two 
quarts of water, putting the whole in an earthenware dish as the iron will rust any metal receptacle.

\section{INDIGESTION.}

The bird refuses to eat; throws up what it has eaten; sour smelling water runs from the crop when the bird is picked up.

Causes.-Overeating; long continuation on the same diet.

Treatment. - Change of diet; give hemp seed liberally; put hypo-sulphate of soda in drinking water. Tablespoonful of saturated solution to one pint of water. (Yive Federation Specific No. 6, and Federation Pill No. I is often useful when in this condition.

\section{INFERTILITY.}

Where the male bird does not impregnate the eggs.

Causes.-Inbreeding; lack of proper tone to the system and confinement. Infertility of the male is determined by examination of the egg on the fifth or sixth day. To examine the eggs hold the egg in the thumb and finger up to a lighted lamp, or the sunlight. If you find what resembles a spider, a dark small spot with blood vessels running from both sides of the center, you may be sure the egg is fortile. Should the egg prove infertile, throw them away, and the birds will usually lay again within ten days.

It is not always a good plan to do this more than once in succession, as it is likely to cause the hen to become barren.

If, on examining the eggs of the second nest, they prove to be infertile, you had better put in one or two fertile eggs that were laid on the same date and allow the birds to hatch them. This method will frequently bring the birds fertile. Sometimes changing the male to another hen will prove a great advantage and result favorably. The same treatment is used in barren. ness of the hen.

\section{JOINT DISEASE OP THE WING.}

Resembles white swelling: joint becomes swelled and inflamed; wing droops.

Causes. - Injury while fighting or flying; sometimes from no apparent cause. Feeding of too rich food may cause it. Inbreeding; also congenital.

Treatment.-Isolate the bird; pluck the feathers from the joint; apply tincture of iodine every third day. Give Federation Specific No. I.

\section{PARALYSIS.}

Inability of the bird to use its legs; it may be partial, progressive and complete.

Causes. - Inbreeding; feeding of too rich food for an indefinite time; it frequently makes its appearance after a cold.

Partial Paralysis. - There may be only one leg paralyzed at first and as the disease progresses both legs become involved and the bird is perfectly helpless. 
When the disease progresses in spite of your remedies, you had better destroy the bird to relieve its misery.

Besides your remedies, you will have to hand-feed the bird.

Treatment.-Give Federation Specific No. I for the first day, follow with Federation Specific No. 2 three times daily.

$$
\text { PULLING THE TAILS OF BIRDS. }
$$

This practice seems to be universal with breeders for a great many years past - the reasons none could tell only that someone else told him to do it. I firmly believe it sets up an irritation, for I have seen birds, after having their tails pulled, rush about as soon as released and hunt for food, and devour it greedily.

In reading a late number of a pigeon paper. I found the following recommended:

"If the bird is dumpy, examine the oil gland at the base at the tail. If yellow and swollen, pull out the tail feathers and squeeze out the puss from the gland. This seems a better plan, except in Maltese Hen Pigeons, as pulling their tails will cause a long growth, which is undesirable. Would recommend close cropping."

\section{ROUP.}

Coughing; sneezing; water discharge from the nostrils; swelling about the eyes, with loss of sight and no passages. and nose.

The discharge is often bad smelling, both from the throat

Ulcers will be found in the throat, appearing like a fungus; loss of appetite; rapid emaciation; loss of strength.

Causes. - Infection; also may follow a bad cold if neglected.

Treatment. - While a majority of fanciers of both pigeons and poultry use a liberal amount of ax, dipping the bead in coal oil is recommended by some and has cured in the early stage. Give Federation Specific No. 4, where there is a stringy glutinous discharge, and Federation Specific No. 6 where there is a rattling in the throat, heard as the bird breathes.

SORE RECTUM.

The rectum protrudes-a scabby collection of excrement and a serious exudation, which, when removed, bleeds quite profusely, leaving a raw surface underneath. Ineffectual straining to move the bowels - the bird will be seen picking the rectum with its bill.

Causes.-Cold; it also may follow an attack of canker.

Treatment. - Removal of the scab; bathing the parts with a weak solution of Hamamelis in water I to $20 . \quad$ Give Federation Spec. No. 7 .

\section{SORE EYES.}

Eyes, are swollen watery and red; inflamed. You will frequently see a cluster of bubbles at one corner of the eye. Loss of sight is often noticed; it may be infectious. 
Causes.-Cold; injury; overfeerling; too crowded quarters.

Treatment. - 3 per cent solution boracic acid dropped in the eye twice a day; bathing the eyes in Hamamelis water I to 20. Federation Spec. No, 1 .

WORMS.

Where you fail to detect anything the matter with the bird, and it still acts badly, rest assured it has worms.

Treatment. - Give small pieces of garlic the size of a pea* repeat next day. Make also a few pellets of cayenne pepper soaked in bread. Give this to the bird one every two hours for half a day. This will strengthen it and facilitate the expulsion of the worms.

We have not taken up the subject of fancy pigeons in this chapter, although many ranches are run to breeding fancy pigeons solely. Plans given will do as well for the fancy stock as for the market squab breeder and we refer our readers to a Pigeon. Standard for descriptions of all varieties. 


FEB 141907 

LIBRARY OF CONGRESS

||||||||||||||||||||||| |||||||||||||||||||

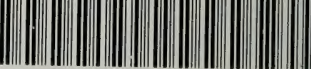

0002840007 A 